\title{
The search for radio emission from the exoplanetary systems 55 Cancri, $v$ Andromedae, and $\tau$ Boötis using LOFAR beam-formed observations
}

\author{
Jake D. Turner ${ }^{1,2}$, Philippe Zarka ${ }^{3,4}$, Jean-Mathias Grießmeier ${ }^{3,5}$, Joseph Lazio ${ }^{6}$, Baptiste Cecconi ${ }^{3,4}$, \\ J. Emilio Enriquez ${ }^{7,8}$, Julien N. Girard ${ }^{9,10}$, Ray Jayawardhana ${ }^{1}$, Laurent Lamy ${ }^{4}$,
} Jonathan D. Nichols ${ }^{11}$, and Imke de Pater ${ }^{12}$

\author{
${ }^{1}$ Department of Astronomy and Carl Sagan Institute, Cornell University, Ithaca, NY, USA \\ e-mail: jaketurner@cornell.edu \\ 2 Department of Astronomy, University of Virginia, Charlottesville, VA, USA \\ ${ }^{3}$ Station de Radioastronomie de Nançay, Observatoire de Paris, PSL Research University, CNRS, Univ. Orléans, OSUC, 18330 \\ Nançay, France \\ ${ }^{4}$ LESIA, Observatoire de Paris, CNRS, PSL, Meudon, France \\ ${ }^{5}$ Laboratoire de Physique et Chimie de l'Environnement et de l'Espace (LPC2E) Université d'Orléans/CNRS, Orléans, France \\ ${ }^{6}$ Jet Propulsion Laboratory, California Institute of Technology, Pasadena, CA, USA \\ ${ }^{7}$ Department of Astronomy, University of California, Berkeley, 501 Campbell Hall \#3411, Berkeley, CA 94720, USA \\ ${ }^{8}$ Department of Astrophysics/IMAPP, Radboud University, PO Box 9010, 6500 GL Nijmegen, The Netherlands \\ ${ }^{9}$ Department of Physics and Electronics, Rhodes University, PO Box 94, Grahamstown 6140, South Africa \\ ${ }^{10}$ AIM, CEA, CNRS, Université Paris-Saclay, Université Paris Diderot, Sorbonne Paris Cité, 91191 Gif-sur-Yvette, France \\ ${ }^{11}$ Department of Physics and Astronomy, University of Leicester, Leicester, UK \\ 12 Department of Astronomy, University of California at Berkeley, Berkeley, CA, USA
}

Received 27 November 2019 / Accepted 22 October 2020

\begin{abstract}
Context. The detection of radio emissions from exoplanets will open up a vibrant new research field. Observing planetary auroral radio emission is the most promising method to detect exoplanetary magnetic fields, the knowledge of which will provide valuable insights into the planet's interior structure, atmospheric escape, and habitability.

Aims. We present LOFAR (LOw-Frequency ARray) Low Band Antenna (LBA: 10-90 MHz) circularly polarized beamformed observations of the exoplanetary systems 55 Cancri, $v$ Andromedae, and $\tau$ Boötis. All three systems are predicted to be good candidates to search for exoplanetary radio emission.

Methods. We applied the BOREALIS pipeline that we have developed to mitigate radio frequency interference and searched for both slowly varying and bursty radio emission. Our pipeline has previously been quantitatively benchmarked on attenuated Jupiter radio emission.

Results. We tentatively detect circularly polarized bursty emission from the $\tau$ Boötis system in the range 14-21 MHz with a flux density of $\sim 890 \mathrm{mJy}$ and with a statistical significance of $\sim 3 \sigma$. For this detection, we do not see any signal in the OFF-beams, and we do not find any potential causes which might cause false positives. We also tentatively detect slowly variable circularly polarized emission from $\tau$ Boötis in the range $21-30 \mathrm{MHz}$ with a flux density of $\sim 400 \mathrm{mJy}$ and with a statistical significance of $>8 \sigma$. The slow emission is structured in the time-frequency plane and shows an excess in the ON-beam with respect to the two simultaneous OFF-beams. While the bursty emission seems rather robust, close examination casts some doubts on the reality of the slowly varying signal. We discuss in detail all the arguments for and against an actual detection, and derive methodological tests that will also apply to future searches. Furthermore, a $\sim 2 \sigma$ marginal signal is found from the $v$ Andromedae system in one observation of bursty emission in the range 14-38 MHz and no signal is detected from the 55 Cancri system, on which we placed a $3 \sigma$ upper limit of 73 mJy for the flux density at the time of the observation.

Conclusions. Assuming the detected signals are real, we discuss their potential origin. Their source probably is the $\tau$ Boötis planetary system, and a possible explanation is radio emission from the exoplanet $\tau$ Boötis b via the cyclotron maser mechanism. Assuming a planetary origin, we derived limits for the planetary polar surface magnetic field strength, finding values compatible with theoretical predictions. Further observations with LOFAR-LBA and other low-frequency telescopes, such as NenuFAR or UTR-2, are required to confirm this possible first detection of an exoplanetary radio signal.
\end{abstract}

Key words. planets and satellites: magnetic fields - radio continuum: planetary systems - magnetic fields planet-star interactions - planets and satellites: aurorae - planets and satellites: gaseous planets

\section{Introduction}

The direct detection of exoplanetary magnetic fields has been elusive despite decades of searching. All the planets in our
Solar System, except Venus, have or used to have a magnetic field (Stevenson 2003), and theoretical scaling laws predict that many exoplanets might have one as well (e.g., SánchezLavega 2004; Grießmeier et al. 2007a; Christensen et al. 2009). 
Measuring the magnetic field of an exoplanet will give valuable information to constrain its interior structure (composition and thermal state), its atmospheric escape, and the nature of any star-planet interaction (Hess \& Zarka 2011; Zarka et al. 2015; Grießmeier 2015, 2017; Lazio et al. 2016, 2019; Lazio 2018; Griessmeier 2018; Zarka 2018). Historically, some of the first constraints on the interior structure of the Solar System gas giants came from the knowledge that they had magnetic fields (Hubbard \& Smoluchowski 1973). Magnetic drag caused by an exoplanet's magnetic field on its atmosphere (e.g., Perna et al. 2010; Rauscher \& Menou 2013; Rogers \& Komacek 2014) could be an important factor for the atmosphere's dynamics and evolution, and it could contribute to the anomalously large radii of hot Jupiters (Laughlin 2018). Additionally, the magnetic field of Earth-like exoplanets might contribute to their sustained habitability by deflecting energetic stellar wind particles and cosmic rays (e.g., Grießmeier et al. 2005a, 2009, 2015, 2016; Lammer et al. 2009; Kasting 2010; Owen \& Adams 2014; Lazio et al. 2010a, 2016; McIntyre et al. 2019).

Many methods have been proposed to study the magnetic fields of exoplanets. A full description of all available methods (excluding the recent method described by Oklopčić et al. 2020 using spectropolarimetric transits of the helium line) can be found in Grießmeier (2015). The methods most extensively discussed in the literature are observations of radio emission (Farrell et al. 1999; Zarka et al. 2001; Zarka 2007), optical signatures of star-planet interactions (SPI; Cuntz et al. 2000; Shkolnik et al. 2003, 2005, 2008; Cauley et al. 2019), and near-ultraviolet light curve asymmetries (Vidotto et al. 2010a, 2011; Llama et al. 2011). The latter two methods have many astrophysical scenarios that can create false-positives (e.g., Preusse et al. 2006; Lai et al. 2010; Kopp et al. 2011; Miller et al. 2012, 2015; Bisikalo et al. 2013; Saur et al. 2013; Alexander et al. 2016; Kislyakova et al. 2016; Turner et al. 2016a; Gurumath et al. 2018; Route 2019). By contrast, radio observation can constrain the magnetic field amplitude directly, without invoking complex model assumptions, and is less susceptible to false positives (Grießmeier 2015). In this paper, we focus on the detection of exoplanetary magnetic fields via radio emission.

All the magnetized planets and moons in our Solar System emit or induce radio emissions via the Cyclotron Maser Instability (CMI) mechanism (Wu \& Lee 1979; Zarka 1998; Treumann 2006). The first proof and measurement of Jupiter's magnetic field, the first measured magnetic field of a planet other than Earth, came from observing its decametric radio emission (Burke \& Franklin 1955). Planetary CMI radio emission is caused by electrons accelerated to energies of several $\mathrm{keV}$ by the interaction of the stellar wind or coronal mass ejections with the magnetosphere or by acceleration processes inside the magnetosphere, resulting from magnetosphereionosphere or magnetosphere-satellite coupling (Cowley et al. 2003; Grießmeier et al. 2007b; Zarka 2007, 2018; Grießmeier 2017). CMI emission is highly circularly polarized, beamed, and time-variable (e.g., Zarka 1998; Zarka et al. 2004). It is produced at the local electron cyclotron frequency (or gyrofrequency) in the source region; its spectrum sharply drops off at a maximum gyrofrequency $v_{\mathrm{g}}$, which is determined by the maximum magnetic field $B_{\mathrm{p}}$ near the planetary surface, as $v_{\mathrm{g}}[\mathrm{MHz}]=$ $2.8 \times B_{\mathrm{p}}[\mathrm{G}]$ (Farrell et al. 1999). This sharp drop-off can also be seen, such as, in Fig. 1.

Recent reviews by Zarka et al. (2015) and Grießmeier (2015, 2017) summarize the observational campaigns and theoretical work concerning radio emission from exoplanets. Following several seminal works (Winglee et al. 1986; Zarka et al. 1997,

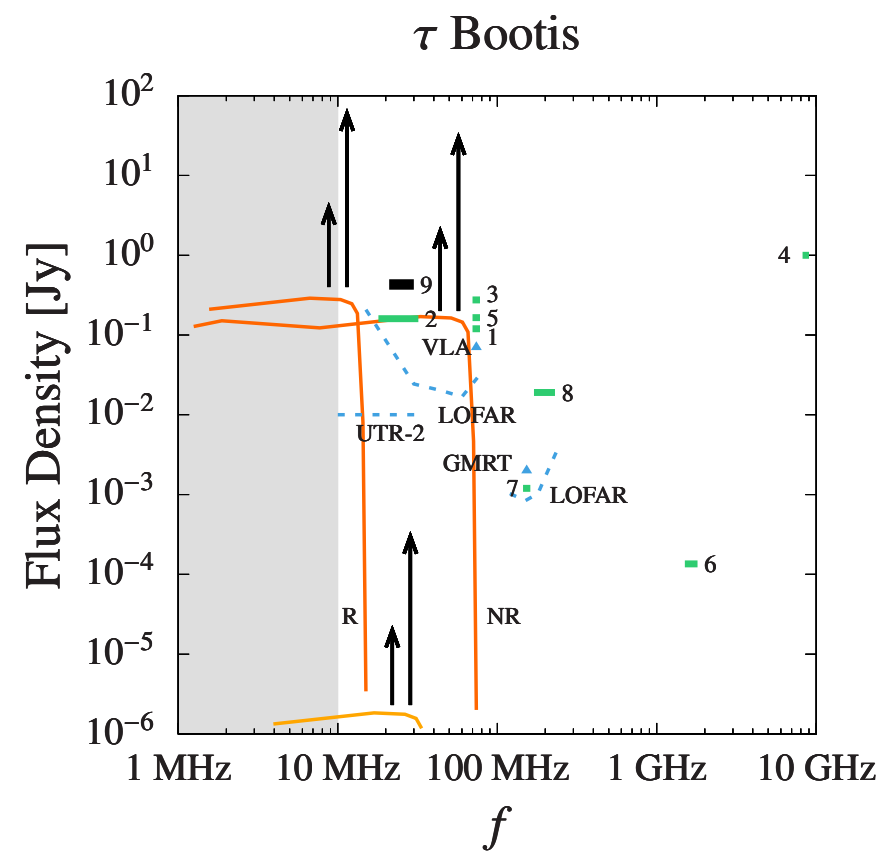

Fig. 1. Predictions and observations for the exoplanet $\tau$ Boötis b. Gray area: emission below $10 \mathrm{MHz}$ is not detectable for ground-based observations (ionospheric cutoff). Lower solid line (light orange): typical spectrum of Jupiter's radio emissions at $15.6 \mathrm{pc}$ distance. Two upper solid line (orange): Jupiter's radio emission scaled for values expected for $\tau$ Boötis b according to models $\mathrm{R}$ (left) and NR (right) from Grießmeier (2017). For Jupiter, the radio flux increases during periods of high activity (frequently by one order of magnitude, and exceptionally by two orders of magnitude, see e.g. Zarka et al. 2004; Zarka 2004); the same variability is assumed for exoplanetary emission, as indicated by the vertical black arrows. Dashed lines and triangles (light blue) show the theoretical sensitivity limit of the radio-telescopes UTR-2, LOFAR (low band), VLA, LOFAR (high band), and GMRT (for $1 \mathrm{~h}$ of integration time and a bandwidth of $4 \mathrm{MHz}$, or an equivalent combination). Numbered lines and points (green): sensitivity achieved in previous observations of $\tau$ Boötis. Care must be taken when comparing the theoretical sensitivity limit to previous observations. The sensitivity limits of the radio telescopes as well as most upper limits were calculated for continuous emission, whereas the values for observations 2 and 9 take into account the expected "bursty" nature of the emission. References: (1) Farrell et al. (2003); (2) Ryabov et al. (2004); (3) Lazio et al. (2004); (4) Shiratori et al. (2006); (5) Lazio \& Farrell (2007); (6) Stroe et al. (2012); (7) Hallinan et al. (2013); (8) Lynch et al. (2018); (9) this article, Sect. 5.1

2001; Farrell et al. 1999; Zarka 2007), an extensive body of theoretical work has been published (e.g., de Pater 2000; Farrell et al. 2004; Lazio et al. 2004; Stevens 2005; Grießmeier et al. 2005b, 2007b,a; Jardine \& Collier Cameron 2008; Vidotto et al. 2010b, 2012, 2015; Hess \& Zarka 2011; Nichols 2011, 2012; See et al. 2015; Nichols \& Milan 2016; Fujii et al. 2016; Weber et al. 2017a,b, 2018; Lynch et al. 2018; Zarka 2018; Wang \& Loeb 2019; Kavanagh et al. 2019; Shiohira et al. 2020).

One of the goals of these theoretical studies is to predict the radio flux and frequency of emission as observed from Earth. However, these predictions are hardly more than educated guesses, with associated uncertainties estimated as one order of magnitude for the flux density and a factor of 2-3 for the maximum emission frequency (Grießmeier et al. 2007a). For example, different rotational-independent and rotationaldependent scaling laws have been employed to predict exoplanetary magnetic-field strengths (e.g., Sánchez-Lavega 2004; 
Grießmeier et al. 2007a; Christensen et al. 2009; Reiners \& Christensen 2010; Grießmeier 2017 - see Christensen 2010 for an overview of all scaling laws in the literature). Similarly, the Radiometric Bode's law, successfully used to predict the radio fluxes from Uranus and Neptune (Desch \& Kaiser 1984; Warwick et al. 1986, 1989; Desch 1988; Million \& Goertz 1988), is often used to estimate the intensity of emission from exoplanets (e.g., Farrell et al. 1999; Lazio et al. 2004), especially in its Radio-Magnetic version (Zarka 2007, 2018; Grießmeier et al. 2007a; Zarka et al. 2018). In particular, using a rotationaldependent scaling law Grießmeier (2017) finds 15 exoplanets with flux densities potentially above the theoretical detection limit Turner et al. (2017a) derived for the beamformed mode of the LOw-Frequency ARray (LOFAR; van Haarlem et al. 2013).

In parallel to these theoretical studies, a number of groundbased observations have been conducted to find radio emission from exoplanets, most of which have resulted in clear nondetections (Yantis et al. 1977; Winglee et al. 1986; Zarka et al. 1997; Bastian et al. 2000; Farrell et al. 2003; Ryabov et al. 2004; Shiratori et al. 2006; George \& Stevens 2007; Lazio \& Farrell 2007; Smith et al. 2009; Lecavelier Des Etangs et al. 2009, 2011; Lazio et al. 2010a,b; Stroe et al. 2012; Hallinan et al. 2013; Murphy et al. 2015; Lynch et al. 2017, 2018; Turner et al. 2017a; O'Gorman et al. 2018; Lenc et al. 2018; Green \& Madhusudhan 2021; Narang et al. 2021; de Gasperin et al. 2020). Most of these studies have involved imaging observations and only span a small fraction of the planetary orbit. A number of possible reasons could account for the non-detections (see, e.g., Bastian et al. 2000; Hallinan et al. 2013; Zarka et al. 2015; Grießmeier 2015, 2017): (1) the observations were not sensitive enough, (2) the planetary magnetic field is not strong enough for emission at the observed frequencies, (3) the Earth was outside the beaming pattern of the radio emission at the time of the observations (Hess \& Zarka 2011), and (4) CMI quenching due to the plasma frequency of the planet's ionosphere being greater than the cyclotron frequency (Grießmeier et al. 2007a; Weber et al. 2017a,b, 2018; Lamy et al. 2018).

There have been a few tentative detections (Lecavelier des Etangs et al. 2013; Sirothia et al. 2014; Vasylieva 2015; Bastian et al. 2018) but none of these have been confirmed by followup observations. The recent detection of $8-12 \mathrm{GHz}$ emission from SIMP0136 is the first radio signal of an object near the brown dwarf and planetary boundary $\left(12.7 \pm 1.0 M_{\mathrm{Jup}}\right.$; Saumon $\&$ Marley 2008) and opens up the possibility of detecting freefloating planets (Kao et al. 2016, 2018). Additionally, V830 $\tau$ is the first non-degenerate exoplanet host-star detected to emit radio emission (Bower et al. 2016) and continued radio monitoring of the system may allow for the detection of star-planet interactions (see Loh et al. 2017, and in prep.). Recent LOFAR observations from the LoTSS survey (Shimwell et al. 2017, 2019) revealed low-frequency radio emission from the M-dwarf GJ 1151 in the range 120-167 MHz, suggested to be caused by the interaction with a close-in Earth-size planet (Vedantham et al. 2020; Pope et al. 2020; Foster et al. 2020).

In this study, we analyze LOFAR Low Band Antenna (LBA) beamformed observations for three exoplanetary systems (55 Cancri, $v$ Andromedae and $\tau$ Boötis). Our data are the first beamformed observations of exoplanets performed with LOFAR. This work extends the preliminary analysis of the LOFAR observation of 55 Cnc published in Turner et al. (2017a).

In this paper, Sect. 2 gives relevant information on the three planetary systems we observed. Sections 3 and 4 describe the observations and data processing, respectively. The analysis of individual observations can be found in Sect. 5. The implications of our tentative detections are discussed in Sect. 6. Section 7 contains conclusions and suggestions for future steps. Extensive supporting material is presented in the appendices.

\section{Target selection}

In this study, we analyze LOFAR-LBA beamformed observations of the planetary systems 55 Cancri, $v$ Andromedae, and $\tau$ Boötis. Based on their proximity to the Solar System, the stellar age, the estimated planetary mass, and the small orbital distance of the planet, these systems were predicted to be good candidates to search for radio emission (Grießmeier et al. 2007a; Grießmeier 2017). All three systems are also considered prime candidates for the search for star-planet interactions (Shkolnik et al. 2005, 2008; Folsom et al. 2020).

The relevant parameters of the planets and their host stars are summarised in Table 1. The values for the expected emission frequency and the expected radio flux are taken from Grießmeier (2017), the data of which are also available electronically at $\mathrm{CDS}^{1}$. We estimate the uncertainties of $v_{\max }$ to be factor 2-3 and the uncertainties on $\Phi_{\max }$ to be approximately one order of magnitude (Grießmeier et al. 2007a). For the magnetic field estimate (required for the calculation of both $v_{\max }$ and $\Phi_{\max }$ ), we have explored two different models. In model NR, the planetary rotation does not have any influence on the planetary magnetic field. In model R, the planetary rotation has an influence on the planetary magnetic field (via tidal locking of the planet with its parent star). Details are given in Grießmeier (2017).

An observation campaign makes sense if the expected emission frequency is above the terrestrial ionospheric cutoff $(\sim 10 \mathrm{MHz})$ and if the expected flux density is higher than the telecope sensitivity. Turner et al. (2019, hereafter T19) estimate that the sensitivity of LOFAR is $\sim 50 \mathrm{mJy}$ for circularly polarized flux (Stokes $V$ ) in integrations of $2 \mathrm{~min}$ and $10 \mathrm{MHz}$. In the following, we compare these numbers to the expected values for the three observed systems.

55 Cancri (55 Cnc). The G8V star 55 Cancri A hosts one of the first known exoplanets; today, five planets are known in this system, and more are expected to exist. The star has a visual binary companion of spectral type $\mathrm{M} 4.5 \mathrm{~V}, \rho^{1} \mathrm{Cnc} \mathrm{B}$, with a projected distance of $1100 \mathrm{AU}$. The two stars are expected to be gravitationally bound (Fischer 2018). For this system, two planets are interesting with respect to radio emission, namely $55 \mathrm{Cnc} \mathrm{b}$ and $55 \mathrm{Cnc}$ e. If the planetary magnetic field does strongly depend on planetary rotation (model R), the emission of $55 \mathrm{Cnc} b$ is below the ionospheric cutoff, but not the emission from $55 \mathrm{Cnc}$ e. If the planetary magnetic field does not strongly depend on planetary rotation (model NR), both planets have emission above the ionospheric cutoff. The expected flux density for $55 \mathrm{Cnc} \mathrm{b}$ is low, but the estimate for $55 \mathrm{Cnc}$ e is above the LOFAR detection threshold. As an added benefit, the orbital period of $55 \mathrm{Cnc}$ e (0.74 days) can be easily covered with a few observations. Good orbital coverage is important if emission is only active for certain orbital phases, which is expected. Also, $55 \mathrm{Cnc}$ e is a transiting planet; in the case of detected radio emission, secondary transits are a good way to check a planetary origin of the radio emission. In the case of a detection, flares from the host star (55 $\mathrm{Cnc} \mathrm{A})$ and its binary companion (55 $\mathrm{Cnc}$ B) will have to be ruled out as potential causes.

ftp://cdsarc.u-strasbg.fr/pub/cats/VI/151/ 
Table 1. Stellar and planetary parameters for the exoplanets observed in this study.

\begin{tabular}{lccccc}
\hline \hline & Units & $55 \mathrm{Cnc} \mathrm{b}^{2}$ & $55 \mathrm{Cnc}$ & $v$ And b & $\tau$ Boo b \\
\hline$*$ type & & ${\mathrm{G} 8 \mathrm{~V}^{(a)}}^{(a)}$ & ${\mathrm{G} 8 \mathrm{~V}^{(a)}}^{(a)}$ & $\mathrm{F9V}$ & $\mathrm{F7V}$ \\
$d$ & {$[\mathrm{pc}]$} & $12.5^{(b)}$ & $12.5^{(b)}$ & $13.5^{(b)}$ & $15.6^{(b)}$ \\
$t_{\star}$ & {$[\mathrm{Gyr}]$} & $10.2 \pm 2.2^{(a)}$ & $10.2 \pm 2.2^{(a)}$ & $3.8 \pm 1^{(b)}$ & $1.0 \pm 0.6^{(b)}$ \\
\hline$a$ & {$[\mathrm{AU}]$} & $0.114^{(a)}$ & $0.0156^{(a)}$ & $0.057^{(c)}$ & $0.0462^{(c)}$ \\
$M_{\mathrm{p}}$ & {$\left[M_{\mathrm{J}}\right]$} & $0.81^{(a)}$ & $0.04^{(a)}$ & $\geq 0.68^{(c)}$ & $\geq 3.87^{(c)}$ \\
$R_{\mathrm{p}}$ & {$\left[R_{\mathrm{J}}\right]$} & unknown $^{(a)}$ & $0.194^{(a)}$ & unknown & unknown \\
\hline$v_{\max }^{N R}$ & {$[\mathrm{MHz}]$} & $20^{(d)}$ & $30^{(d)}$ & $14^{(d)}$ & $74^{(d)}$ \\
$\Phi_{\max }^{N R}$ & {$[\mathrm{mJy}]$} & $2.9^{(d)}$ & $150^{(d)}$ & $75^{(d)}$ & $170^{(d)}$ \\
\hline$v_{\max }^{R}$ & {$[\mathrm{MHz}]$} & $3.3^{(d)}$ & $19^{(d)}$ & $2.2^{(d)}$ & $15^{(d)}$ \\
$\Phi_{\max }^{R}$ & {$[\mathrm{mJy}]$} & $5.3^{(d)}$ & $170^{(d)}$ & $140^{(d)}$ & $290^{(d)}$ \\
\hline
\end{tabular}

Notes. Row 2: spectral type of host star. Row 3: stellar distance. Row 4: stellar age. Row 5: orbital distance. Row 6: planetary mass. Row 7: planetary radius. Row 8: expected maximum emission frequency (under the hypothesis planetary rotation does not have any influence on the planetary magnetic field). Row 9: maximum expected radio flux density at Earth (under the hypothesis planetary rotation does not have any influence on the planetary magnetic field). Row 10: expected maximum emission frequency (under the hypothesis planetary rotation has a strong influence on the planetary magnetic field). Row 11: maximum expected radio flux density at Earth (under the hypothesis planetary rotation has a strong influence on the planetary magnetic field).

References. ${ }^{(a)}$ Fischer (2018), ${ }^{(b)}$ Fuhrmann et al. (1998), ${ }^{(c)}$ Butler et al. (1997), ${ }^{(d)}$ Grießmeier (2017).

$v$ Andromedae $(v$ And). $\quad v$ Andromedae A is a F9V star with a stellar binary companion, Andromedae B, at a projected distance of $\sim 700$ AU from the primary star. The binary companion $v$ Andromedae $\mathrm{B}$ is of spectral type $\mathrm{M} 4.5 \mathrm{~V}$ and has been detected in X-rays (Poppenhaeger \& Wolk 2014). For model R, the estimated emission frequency is below the ionospheric cutoff, but in model NR, the expected planetary radio emission extends to detectable frequencies. The expected flux density is above the LOFAR detection threshold. Flares from the host star and its binary companion will have to be ruled out as potential causes in the case of a detection.

$\tau$ Boötis ( $\tau$ Boo). $\tau$ Boötis A is a hot and young F7V star. Its binary companion $\tau$ Boötis B is of spectral type $\mathrm{M} 3 \mathrm{~V}$; it is on a highly eccentric orbit $(e=0.87)$ with a semimajor axis of 220 AU (Justesen \& Albrecht 2019). For model R, the expected planetary radio emission only slightly exceeds the ionospheric cutoff limit. Nominally, the flux exceeds the detection threshold, but the estimate of $50 \mathrm{mJy}$ does not take into account the reduced sensitivity of LOFAR at $15 \mathrm{MHz}$. However, for model NR the emission extends to detectable frequencies, and the expected flux density is above the detection threshold. As for the two other systems, flares from the host star and its binary companion will have to be ruled out as potential causes in the case of a detection.

Figure 1 compares the predicted radio flux for $\tau$ Boo b to the sensitivity achieved in previous observations. This figure makes the motivation behind our observations tangible: radio fluxes predicted by at least some of the models are compatible with the theoretical sensitivity limits of several radio-telescopes. In particular, according to the model NR, radio emission from $\tau$ Boo b should be detectable by UTR-2 and LOFAR (low band), and possibly by the VLA.

\section{Observations}

Our observations were taken with the LOw-Frequency ARray (LOFAR; van Haarlem et al. 2013), using its Low Band Antenna (LBA, 10-90 MHz) in beamformed mode (Stappers et al. 2011). The setup used for our observations can be found in Table 2.
Table 2. Setup of the LOFAR-LBA beamformed observations.

\begin{tabular}{ccc}
\hline \hline Parameter & Value & Units \\
\hline Array setup & LOFAR Core & \\
Number of stations & 24 & \\
Beams & ON \& 2 OFF & \\
Configuration & LBA outer antennas & \\
Antennas per station & 48 & \\
Minimum frequency & $26^{(a)}$ or $15^{(b)}$ & $\mathrm{MHz}$ \\
Maximum frequency & $74^{(a)}$ or $62^{(b)}$ & $\mathrm{MHz}$ \\
Subbands recorded & 244 & \\
Subband width & 195 & $\mathrm{kHz}$ \\
Channels per subband & 64 & \\
Frequency resolution $(b)$ & 3.05 & $\mathrm{kHz}$ \\
Time resolution $(\tau)$ & 10.5 & $\mathrm{msec}$ \\
Beam diameter $(c)$ & 13.8 & $\mathrm{arcmin}$ \\
Raw sensitivity $(\Delta S)(d)$ & 208 & $\mathrm{Jy}$ \\
Stokes parameters & $I Q U V$ & \\
\hline
\end{tabular}

Notes. ${ }^{(a)}$ Frequency range for the 55 Cnc observations. ${ }^{(b)}$ Frequency range for the $v$ And and $\tau$ Boo observations. ${ }^{(c)}$ Calculated at $30 \mathrm{MHz}$ (van Haarlem et al. 2013). ${ }^{(d)}$ Raw sensitivity of a pixel in the dynamic spectrum, calculated using $\Delta S=S_{\text {sys }} / N \sqrt{n_{\text {pol }} \tau b}$, with $S_{\text {sys }}$ the system equivalent flux density (SEFD) of one LOFAR Core station $(40 \mathrm{kJy}$; van Haarlem et al. 2013), $N$ the number of stations summed, $n_{\mathrm{pol}}$ is the number of polarizations (2), $b$ the frequency resolution, and $\tau$ the time resolution.

The exact dates and times of each observation can be found in Appendix A (Table A.1), which also gives the coordinates used for the $\mathrm{ON}$ and OFF beams (Table A.2).

The data streams from all 24 core stations were summed together during the observations. All the processing steps described in this paper are performed on this summed data product. This means it is not possible to flag out the contribution of any of these stations a posteriori if any station does not operate optimally, the stations were phased up to produce multiple 
simultaneous digital beams within the broader individual station beams: one pointing at the target, and two pointing at different OFF sky positions (used for comparison in the data processing pipeline, see below).

We focus on the Stokes- $V$ data. T19 have shown that this allows one to detect signal that are 1 to 1.5 orders of magnitude weaker than when using Stokes- $I$ data (provided that the signal is circularly polarized, which is expected here). The observations were taken at high temporal and spectral resolutions ( $\tau$ and $b$ ) in order to allow proper RFI mitigation.

As in our previous studies (Turner et al. 2017a, 2019), we compare our on-target beam ("ON-beam") to several simultaneous beams pointing to a nearby location in the sky ("OFF-beam 1 " and "OFF-beam 2"). A fundamental assumption of this method is that the OFF beams provide a good characterization of the ionospheric fluctuations, RFI, and any systematics present in the ON-beam. For that purpose, the OFF beams are well within the station beams $\left(10^{\circ} \mathrm{FWHM}\right.$ at $\left.30 \mathrm{MHz}\right)$, far enough from the ON-beam to not overlap within the beam diameter $\left(13.8^{\prime}\right.$ at $30 \mathrm{MHz}$ ), and also close enough to the ON-beam to be within the ionospheric isoplanatic patch $\left(7^{\circ}\right.$ at $30 \mathrm{MHz}$; Intema et al. 2009 and C. Vogt, priv. comm.).

For this project, 22 exoplanet observations were taken with a total of $89 \mathrm{~h}$, plus 16 observations of B0809+74 totaling $197 \mathrm{~min}$. The observations were all obtained during night-time to avoid contamination by strong RFI. During the observing period May 19-November 22, 2016 the LBA calibration tables were unreadable, resulting in $20 \%$ higher noise (communication from ASTRON Radio Observatory staff). Both the $55 \mathrm{Cnc}$ and $v$ And data are affected by this calibration error.

For geometrical reasons, we expect the emission to be beamed at Earth only for a fraction of the time (e.g., Hess \& Zarka 2011). Assuming the anisotropic beaming is similar to that of Jupiter (Zarka et al. 2004), we would expect the emission to be visible from Earth only $\sim 10 \%$ of the time. Also, the intensity of the radio emission is expected to vary as the planet encounters different plasma conditions (particle density, magnetic field) along the orbit (e.g., Grießmeier et al. 2005b, 2007b; Fares et al. 2009; Vidotto et al. 2012). For these reasons, the observation windows were chosen such that the orbital coverage is as wide as possible in order to maximize the chances to detect beamed emission. The orbital coverage of our observations is shown in Fig. 2. For the observations of $55 \mathrm{Cnc}$ (with a total observing time of $24 \mathrm{~h}$ ), the observation windows can be folded at the orbital period of either $55 \mathrm{Cnc} \mathrm{b}$ (Fig. 2a) or $55 \mathrm{Cnc}$ e (Fig. 2b). For the short-period transiting planet $55 \mathrm{Cnc}$, the full orbit is covered more than once, increasing the chances of catching beamed emission. Also, secondary eclipses constitute a powerful tool: If radio emission is detected, but vanishes during the secondary eclipse, this can be taken as a very strong indication for a planetary origin of the radio emission (e.g., Grießmeier et al. 2005b; Smith et al. 2009; Lecavelier des Etangs et al. 2013) For $55 \mathrm{Cnc} \mathrm{b}$, the orbital period is longer, and the phase coverage is low (9\%). For $v$ And (Fig. 2c), the phase coverage is $40 \%$ with a total observing time of $45 \mathrm{~h}$. Finally, for $\tau$ Boo (Fig. 2d), the phase coverage is $25 \%$ of the orbit with a total observing time of $20 \mathrm{~h}$; we cannot exclude that we have missed radio emission concentrated at specific orbital phases.

\section{Data pipeline}

Based on previous work (Vasylieva 2015; Turner et al. 2017a, 2019), we have developed the BeamfOrmed Radio Emission AnaLysIS (BOREALIS) pipeline for our exoplanetary beam-formed data. It was applied to the both Stokes- $I$ and Stokes- $V$ data-sets of the observations presented in Sect. 3.

This pipeline performs RFI mitigation, empirically determines the time-frequency $(t-f)$ response of the telescope (i.e. the gain), normalizes the data by this $t$ - $f$ function, and rebins the data into larger time and frequency bins. The RFI mitigation combines four different techniques (Offringa et al. 2010, 2012; Offringa 2012; Zakharenko et al. 2013; Vasylieva 2015; and references therein) for optimal efficiency and processing time, following which the data is normalized and rebinned to time-frequency bins of $1 \mathrm{~s} \times 45 \mathrm{kHz}$. The expected thermal noise level for the rebinned data is $6 \mathrm{Jy}$ for each measurement. The resulting corrected Stokes- $V$ dynamic spectrum $\left(V^{\prime}\right.$, defined in Eqs. (9)-(11) of Sect. 4.2.1 in T19) is the input for the post-processing part of the BOREALIS pipeline. An example of a processed dynamic spectrum for each target is displayed in Fig. 3. The fine structure seen in Fig. 3 can be caused by ionospheric scintillation on the Galactic background and some small residual instrumental effects. Additionally, the variable patches seen above $40 \mathrm{MHz}$ are likely produced by LOFARcore's grating lobes. At lower frequencies the grating lobes are below the horizon. The fluctuations in the flux of the OFFbeams are less than $1 \%$ which is consistent with the expected thermal noise error of $\sim 0.5 \%$. We note that the dynamic spectra presented throughout this paper are in units of the system equivalent flux density (SEFD) since the data has been normalized by the $t-f$ response function of the telescope. The SEFD include contributions from both the sky and instrument. The SEFD of LOFAR does change slightly with frequency (see van Haarlem et al. 2013). In this paper we use an average value of $40 \mathrm{kJy}$ for the SEFD of a single LOFAR station, that is to say $1.7 \mathrm{kJy}$ for the full LOFAR core (i.e. the sum of 24 LOFAR stations). We use these units to simplify the display and analysis of the dynamic spectra and convert to Jy when necessary.

The post-processing part of the BOREALIS pipeline is based on a series of observable quantities (labeled Q1a, Q1b, Q2, and Q4a-f). These quantities were introduced in T19; they are also recalled in Appendix B. The quantities Q1a and Q1b represent integrated quantities, namely the frequency-integrated time-series (Q1a) and the time-integrated frequency spectrum (Q1b). Both Q1a and Q1b are designed to find slowly variable emission (with time scales of minutes to hours). Q2 represents the high pass filtered frequency-integrated time-series. It can be displayed as a "scatter plot" comparing a pair of beams (e.g., the $\mathrm{ON}$ and OFF beam) and is designed to find bursty emission (with time scales $<\sim 1 \mathrm{~min}$ ). The quantities Q4a to Q4f provide statistical measures of the bursts identified by the Q2 quantity. When examining Q4a-f, the ON and OFF time series are compared to each other; for this, we introduce the difference curve $\mathrm{Q} 4 \mathrm{f}_{\text {Diff }}=$ $\mathrm{Q} 4 \mathrm{f}(\mathrm{ON})-\mathrm{Q} 4 \mathrm{f}(\mathrm{OFF})$. We then plot this curve against a reference curve computed from 10000 draws of purely Gaussian noise. A more detailed description of each observable can be found in Appendix B, or in T19.

The efficiency of the quantities Q4a to Q4f for the detection of faint bursty emission was compared in T19. This benchmark analysis was based on artificially attenuated radio emission of Jupiter and led to the conclusion that Q4f is the most sensitive observable quantity for faint burst emission. However, exoplanetary radio emission may be different from Jupiter radio emission. Therefore, we examined all the observable quantities in our analysis and not just the most sensitive one indicated by the Jupiter benchmark study (Q4f).

The post-processing is initially performed on the absolute value of the corrected Stokes- $V$ data $\left(\left|V^{\prime}\right|\right)$. In case of a tentative 
(a)

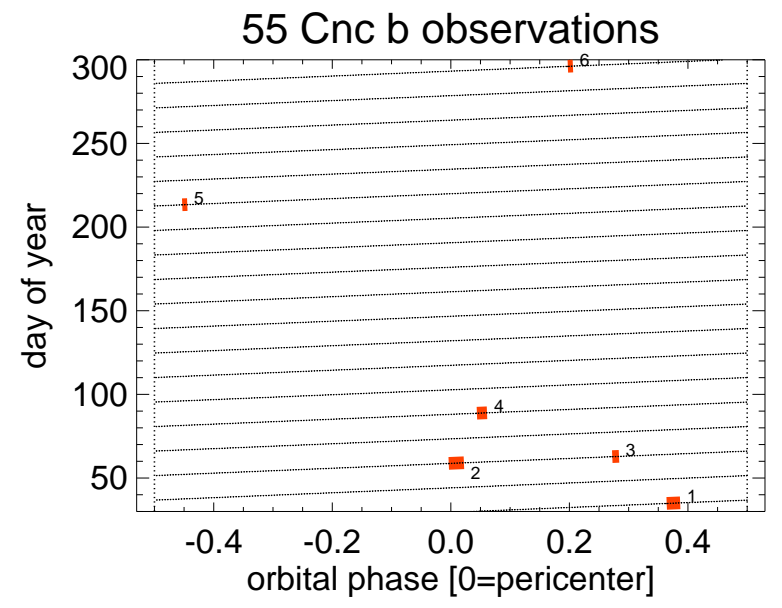

(c)

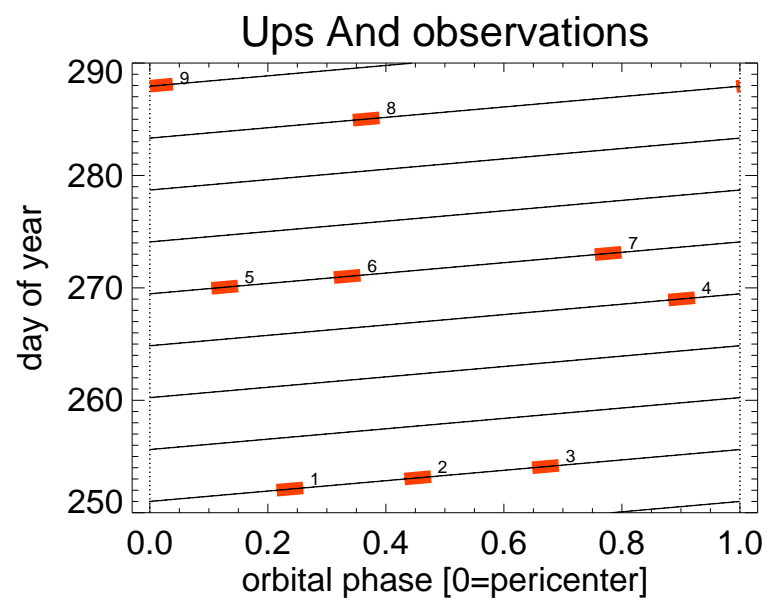

(b)

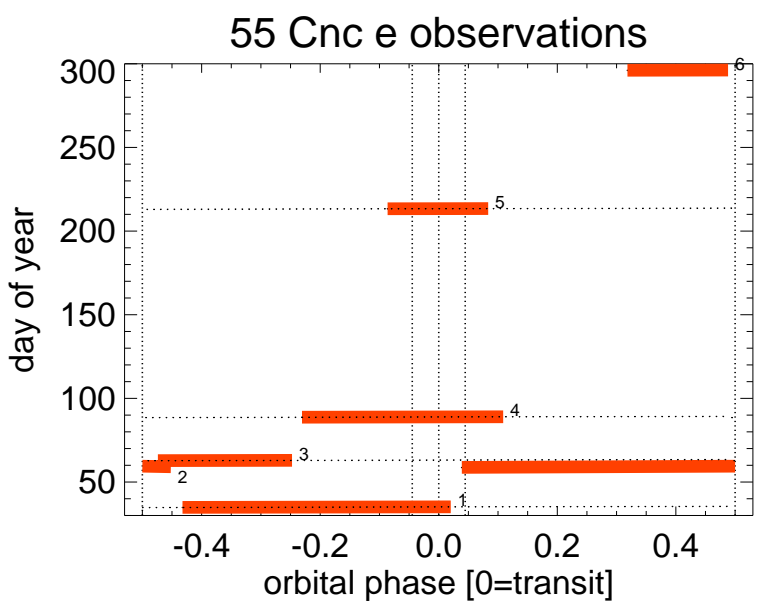

(d)

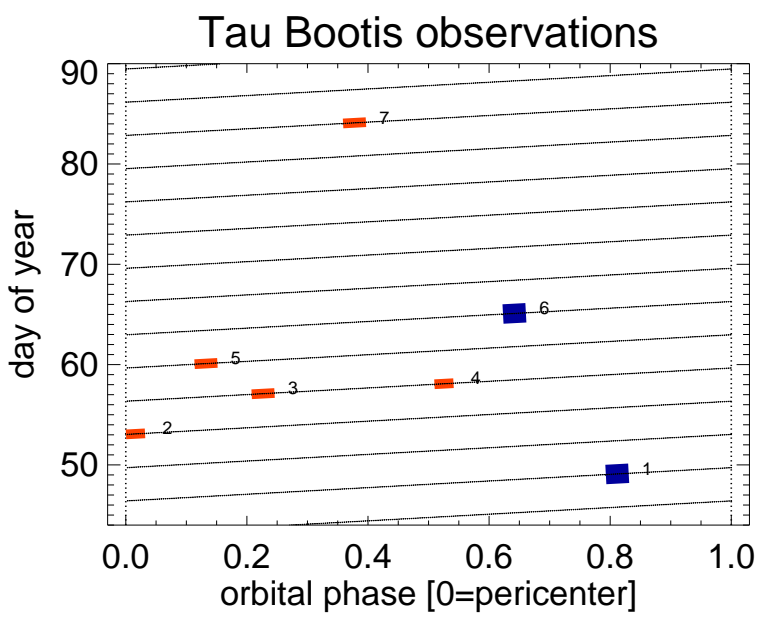

Fig. 2. Orbital phase coverage for the observations of $55 \mathrm{Cnc} \mathrm{b}$ (panel a), $55 \mathrm{Cnc}$ e (panel $b$ ), $v$ And $\mathrm{b}$ (panel c), and $\tau$ Boo $\mathrm{b}$ (panel $d$ ). The numbers representing each date are indicted in Col. 1 of Table A.1. We note that $55 \mathrm{Cnc}$ e is a transiting exoplanet. For 55 Cnc e, only a few planetary rotations are shown. For 55 Cne e, the entire orbit is covered by the observations, whereas the orbital coverage is low for $55 \mathrm{Cnc} b$. Approximately 40 and 25\% of the orbits of $v$ And $\mathrm{b}$ and $\tau$ Boo $\mathrm{b}$ are covered, respectively. The $\tau$ Boo observations with the tentative detections (Sect. 5.1; obs \#1 and \#6) are displayed as large dark-blue rectangles in panel d.

detection, information on whether the polarization of the emission is right-handed or left-handed is obtained by analysing $V^{\prime}$ and $V^{\prime}$ - where,

$V^{\prime}=\left\{\begin{array}{ll}-V^{\prime}, & \text { if } V^{\prime}<0 \\ 0, & \text { otherwise }\end{array}\right.$,

$V^{+}=\left\{\begin{array}{ll}V^{\prime}, & \text { if } V^{\prime}>0 \\ 0, & \text { otherwise }\end{array}\right.$.

The post-processing was performed separately over 3 different frequency ranges depending on the observational setup and target. A summary of the parameters used in the post-processing can be found in Table 3. While the nominal rebin time was $1 \mathrm{~s}$, we also reanalyzed the dataset with a rebin time of 10 seconds to search for longer and weaker bursts of emission. The RFI mitigation step creates a high-resolution mask (at scales $b$ and $\tau$ of Table 2) of flags ( 0 for polluted pixels, 1 for clean ones). Since the processed dynamic spectrum is rebinned, we also have to rebin the flag mask to the same time and frequency resolution. Consequently, the rebinned flag mask contains fractional values between 0.0 and 1.0. In the post-processing we applied a threshold of $90 \%$ on the mask, meaning that that we only retain a bin if $\geq 90 \%$ of its constitutive pixels were clean. We note also that the elliptical correction described in T19 and summarised in Appendix B was systematically applied to the analysis of Q2 and Q4 as this correction allows one to detect fainter signals.

The basic assumption of our analysis is that the OFF beams characterize the noise and systematics that affect the ON-beam well enough to search for a faint signal by comparison (ON vs. OFF) or difference (ON-OFF). As Q4 difference curves often show large fluctuations, we found it absolutely necessary to record and analyze two OFF beams simultaneously with the $\mathrm{ON}$ beam. The analysis of one observation with a given set of processing parameters includes the statistical comparison of the three different combination of beams: ON vs. OFF1, ON vs. OFF2, and OFF1 vs. OFF2. We label a signal as a tentative dection only if an excess appears in the former 2 pairs and not in the latter one. If the OFF1 vs. OFF2 analysis shows an excess (with any sign) then a simultaneous tentative signal in ON vs. OFF is considered as a false positive, and discarded. All the conclusions in this paper are the same when comparing the ON-beam 
(a)

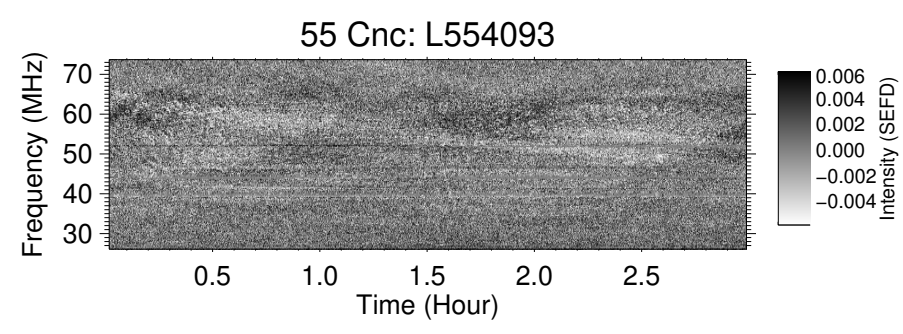

(b)

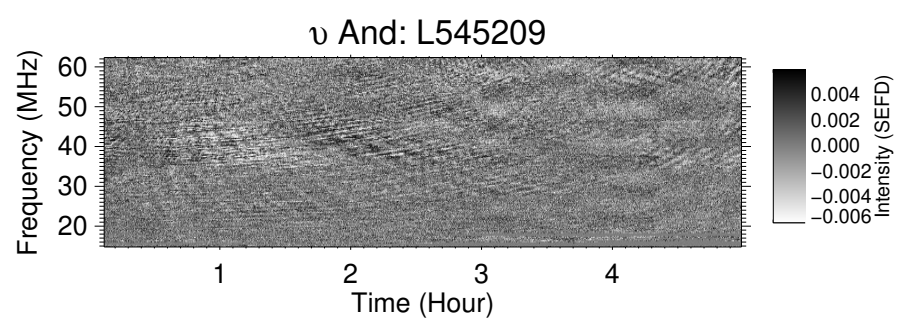

(c)

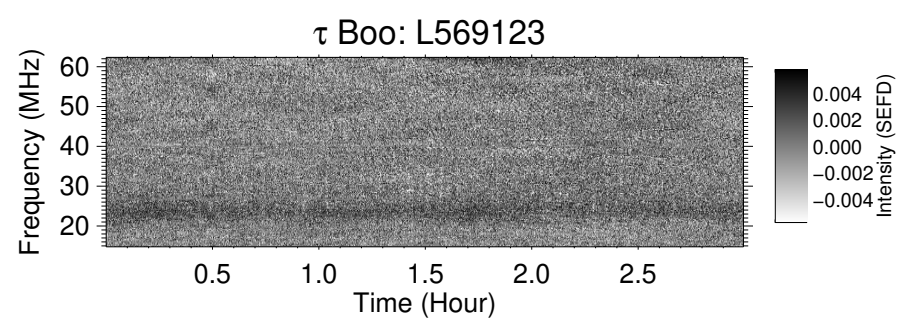

Fig. 3. Examples of processed dynamic spectra in Stokes $V$ (see Sect. 4) for OFF beam 2 of the 55 Cnc observation L554093 (panel a), the $v$ And observation L545209 (panel b), and the $\tau$ Boo observation L569123 (panel c). The dynamic spectra presented are in units of the system equivalent flux density (SEFD) since they have been normalized by the $t-f$ response function of the telescope.

Table 3. Parameters for the post-processing pipeline.

\begin{tabular}{ccc}
\hline \hline Parameter & Value & Units \\
\hline Frequency ranges & 26-74, $26-50,50-74^{(a)}$ & $\mathrm{MHz}$ \\
& $15-62,15-38,38-62^{(b)}$ & $\mathrm{MHz}$ \\
Q1 Time bins $(\delta T)$ & 2 & $\mathrm{~min}$ \\
Q1 Frequency bins $(\delta F)$ & 0.5 & $\mathrm{MHz}$ \\
Q2-Q4 rebin times $(\delta \tau)$ & 1,10 & $\mathrm{~s}$ \\
Mask threshold & 90 & $\%$ \\
Smoothing window & 10 & $\delta \tau$ \\
for high-pass filtering & $1-6$ & $\sigma$ \\
Threshold $(\eta)$ range & & \\
\hline
\end{tabular}

Notes. ${ }^{(a)}$ Frequency ranges for the 55 Cnc observations. ${ }^{(b)}$ Frequency ranges for the $v$ And and $\tau$ Boo observations.

to either of the OFF beams (OFF1 or OFF2). For this reason, we simply refer to the "ON vs. OFF2" comparison as "ON vs. OFF" throughout the rest of the paper.

In total, $\sim 13000$ summary plots were produced, summarizing the analysis of all observations (Table A.1) using the post-processing parameters given in Table 3 . We applied the following criteria defined in T19 for a possible detection: (1) one or several Q4 curves show an excess $\geq 2 \sigma$ statistical significance for the ON-OFF statistics, (2) the same Q4 curves have a shape clearly different from the OFF1-OFF2 curve, and (3) the detection curve remains positive over a large interval of thresholds $\eta>1.5 \sigma$. The way these criteria are implemented in an automated search algorithm is described in Appendix C.

\section{Data analysis and results}

For most of the observations and frequency ranges explored we did not find any excess signal in the ON-beam when compared to the OFF beams. The observables Q1a and Q1b do show some structure, but those structures are identical between the $\mathrm{ON}$ and OFF beam (examples for Q1b are shown in panels $b$ and $d$ of Fig. D.1). Indeed, for all observations (except for observation L570725 of $\tau$ Boo, see Sect. 5.1.2), the curves for the ON and OFF beams are equivalent within $1 \sigma$. We also tested the presence of red noise in the difference time-series (Q1a) for all our nondetections using the time-averaging method (Pont et al. 2006; Turner et al. 2016b, 2017b), and found none. Thus the majority of the difference in the signal between the two beams can be explained purely by Gaussian white noise. We also searched for burst emission using Q4a-f; for almost all observations, the burst statistics were similar between the $\mathrm{ON}$ and OFF beams.

For $\tau$ Boo we found one tentative detection of faint burst emission in observation L569131 and one tentative detection of slowly variable emission in observation L570725, as summarised in Table 4. These tentative detections are examined in detail in Sect. 5.1. We also find a marginal signal of faint burst emission for $v$ And in observation L545197, which is explored more in detail in Sect. 5.2.

\section{1. $\tau$ Boötis}

\subsubsection{Observation L569131 (2017-02-18)}

For $\tau$ Boo, we detect Stokes- $V$ burst emission in the frequency band $15-38 \mathrm{MHz}$ in observation L569131 from the Q4 observables (Fig. 4; Table 4). In particular, Fig. 4 shows an excess of bursts in the ON-beam in Q4f (panel $\mathrm{f}$, curve ON-OFF), whereas both OFF beams show comparable statistics (the curve OFF1OFF2 is almost flat). The burst emission is also seen in $V^{\prime}$ (defined in Eq. (2)), suggesting that the emission is left-hand polarized.

To isolate the frequency range of the signal, we processed a finer grid of frequency ranges (6 $\mathrm{MHz}$ in bandwith) within 15-38 MHz. Performing this test, we find that the majority of the signal is from the $15-21 \mathrm{MHz}$ range. Therefore, we use this frequency range to tentatively deduce physical information about the planet (see Sect. 6.2.1).

We estimate the flux density of this signal using the sensitivity limit derived in T19. In that work, this limit was found to be equal to 1.3 times the LOFAR theoretical sensitivity $\left(\sigma_{\text {LOFAR }}\right)^{2}$ and consisted of 30 data points above $\eta=2 \sigma$ in Q4f. The signal in Q4f for observation L569131 consists of 21 data-points (each binned to a resolution of $1 \mathrm{~s}$ and $24 \mathrm{MHz}$ ) above $\eta=2 \sigma$ level $^{3}$. These points result in a $\mathrm{Q} 4 \mathrm{f}_{\text {Diff }}$ curve above the 2 sigma Gaussian reference curve. The flux in the tentative detection should be near the sensitivity limit since the number of detected points

2 The $1.3 \sigma_{\text {LOFAR }}$ sensitivity limit in Stokes- $V$ is also consistent with the recent finding by Mertens et al. (2020) who use imaging observations.

3 The number of 21 points is not directly visible in the figures; it relates to the underlying Q2 distribution that was used to calculate the observable quantity Q4f. 
Table 4. Summary of detected signals and derived planetary parameters assuming that the emission originates from the planet (see Sect. 6 for a discussion of the arguments for and against a planetary origin for the signals).

\begin{tabular}{ccccccccccc}
\hline \hline $\begin{array}{c}\text { Planet } \\
\text { name }\end{array}$ & $\begin{array}{c}\text { LOFAR } \\
\text { Obs ID }\end{array}$ & $\begin{array}{c}v \\
(\mathrm{MHz})\end{array}$ & $\begin{array}{c}\text { Polarization } \\
(|V|, \mathrm{V}+, \mathrm{V}-)\end{array}$ & Q\# & $\begin{array}{c}\text { Signifi- } \\
\text { cance }(\sigma)\end{array}$ & Figure & $\begin{array}{c}\text { Flux } \\
(\mathrm{mJy})\end{array}$ & $\begin{array}{c}\text { Power } \\
(\mathrm{W})\end{array}$ & $\begin{array}{c}T_{\mathrm{B}} \\
(\mathrm{K})\end{array}$ & $\begin{array}{c}\text { Magnetic } \\
\text { field }(\mathrm{G})\end{array}$ \\
\hline$\tau$ Boo b & L570725 & $21-30$ & $|V|, \mathrm{V}+$ & $\mathrm{Q} 1 \mathrm{a}$ & 6.9 & 5 & $190 \pm 30$ & $6.3 \times 10^{14-15}$ & $4.2 \times 10^{17}$ & $7.5-10.7$ \\
$\tau$ Boo b & L570725 & $21-30$ & $|V|, \mathrm{V}+$ & $\mathrm{Q} 1 \mathrm{~b}$ & 8.6 & 5 & $430 \pm 30$ & $1.4 \times 10^{15-16}$ & $1.0 \times 10^{18}$ & $7.5-10.7$ \\
$\tau$ Boo b & L569131 & $15-21$ & $|V|, \mathrm{V}-$ & $\mathrm{Q} 4 \mathrm{f}$ & 3.2 & 4 & $890_{-500}^{+690}$ & $2.0 \times 10^{15-16}$ & $2.0 \times 10^{18}$ & $5.4-7.5$ \\
\hline
\end{tabular}

Notes. Column 1: planet name. Column 2: LOFAR observation ID. Column 3: frequency range of the detection. Column 4: polarization signature of the emission. Column 5: observable quantity that found the detection. Column 6: statistical significance derived using Gaussian simulations. Column 7: respective figures showing the detected signals. Column 8: estimated flux density of the emission. Column 9: estimated power of the emission. Column 10: brightness temperature of the tentative emission. Column 11: maximum polar surface magnetic field of the planet derived using the maximum frequency, assuming CMI emission.

between the Jupiter observation (T19) and the $\tau$ Boo observation (the present work) are comparable ${ }^{4}$. Also, the uncertainty is similar to that derived in T19.

The theoretical sensitivity limit of LOFAR for broadband bursts is

$\sigma_{\text {LOFAR }}=\frac{S_{\text {Sys }}}{N \sqrt{b \tau}}$,

$\sigma_{\text {LOFAR }}=\frac{40 \mathrm{kJy}}{24 \sqrt{1 \mathrm{~s} \times 6 \mathrm{MHz}}}=680 \mathrm{mJy}$,

where $N$ is the number of stations (24), $b$ is the bandwidth (6 MHz), $\tau$ is the timing resolution ( $1 \mathrm{~s})$, and $S_{\mathrm{Sys}}$ is the station SEFD with a value of $40 \mathrm{kJy}$ (van Haarlem et al. 2013). Using the sensitivity limit of $1.3 \times \sigma_{\text {LOFAR }}$ (T19) and Eq. (4), we find a flux density estimate for the burst emission of $890_{-500}^{+690} \mathrm{mJy}$. In this, we assumed an uncertainty of a quarter of an order of magnitude $\left(10^{0.25}\right)$ for the sensitivity limit (see T19). The uncertainties given here are merely rough estimates; the real values depend on the time and frequency scale of the emission.

To quantity the statistical significance of the detected signal we use the method outlined in T19. The difference of the ON-OFF beam in the observable Q4f $\left(\mathrm{Q}_{4} \mathrm{f}_{\text {Diff }}\right.$ the solid black line in Fig. 4f) is normalized by the $1 \sigma$ Gaussian statistical limit (the lower-positive dashed-line in Fig. 4f) and we calculate its average value $\left(\left\langle\mathrm{Q} 4 \mathrm{f}_{\text {Diff }}\right\rangle\right)$. $\left\langle\mathrm{Q} 4 \mathrm{f}_{\text {Diff }}\right\rangle$ is then compared to the case when the ON and the OFF beams only contain random noise. Using this procedure, we find that the probability of a false positive for obtaining the ON-beam signal is $7 \times 10^{-4}$, equivalent to a $3.2 \sigma$ detection. Finally, we compare these values to those of the two OFF beams (the solid red line in Fig. 4f). For the OFF beams we find a false positive rate of $19 \%$ indicating clearly a non-detection.

To further assess the statistical significance of the detected signal we perform a two-sided Kolmogorov-Smirnov (K-S) test in which we compare the Q4f ON-OFF and OFF1-OFF2 curves. The null hypothesis is that the two curves are drawn from the same parent distribution. Performing the K-S test we find that the probability to reject the null hypothesis (that the two curves are drawn from the same parent distribution) is high with a value of $98 \%$. This test adds to the evidence that the two curves are distinctly different and that this signal is real.

To summarize this analysis: We detect a bursty signal in this observation. This signal is statistically significant. Its potential origin is discussed in Sect. 6.

\footnotetext{
4 The relationship between the signal intensity and the number of points is nonlinear, see Fig. 2c in T19.
}

\subsubsection{Observation L570725 (2017-03-06)}

For $\tau$ Boötis, we also detected slowly variable emission in the range $14-38 \mathrm{MHz}$ in observation L570725. This emission was detected using the dynamic spectra integrated over frequency (time-series, Q1a) and time (integrated spectrum, Q1b) in Stokes- $V$ (Fig. 5; Table 4). The signal is also an outlier when compared to all $N 2$ numbers for all observations (Fig. C.3). The OFF beams are equivalent within $1 \sigma$ for both Q1a and Q1b suggesting that there is an excess signal in the ON beam. Further examination of Q1b determined that the bulk of the ON-beam emission is coming from the range $21-30 \mathrm{MHz}$. We did not find any short-term (1-10 s) burst emission using the observable Q4 for this observation (Fig. E.1). The slow emission feature is seen in $V^{\prime}$ but not in $V^{\prime-}$ (not shown), suggesting that the emission if physical - is right-hand circularly polarized.

The flux density of the signal can be estimated from the ONOFF beam difference (bottom panels in Figs. 5a,b). We find a mean signal of $1.1 \pm 0.2 \times 10^{-4}$ of the theoretical SEFD from Q1a. Since all the emission is coming from the range $21-30 \mathrm{MHz}$ (Fig. 5b), the calculation of Q1a was limited to this range. We find a value of $190 \pm 30 \mathrm{mJy}$ for the time-averaged signal. The quoted error above is the standard deviation of the difference of Q1a for the OFF beams. We find a maximum emitted flux of $430 \pm 30 \mathrm{mJy}$ using the peak spectral flux $\left(\sim 2.5 \times 10^{-4}\right.$ of the SEFD) observed in the difference of Q1b. Again, the error is the standard deviation of the difference of Q1b for the OFF beams.

To quantify the statistical significance of this signal, we use a method similar to the technique outlined in T19 and described thoroughly in Appendix F. We find that the probability of a false positive for obtaining a signal like the one we observe is $2.1 \times 10^{-12}$ for the Q1a(ON)-Q1a(OFF2) (bottom panel in Fig. 5a) and $1 \times 10^{-18}$ for Q1b(ON)-Q1b(OFF2) (bottom panel in Fig. 5b). This corresponds to a statistically significant signal of $6.9 \sigma$ and $8.6 \sigma$, respectively. For the OFF-beams we find that the false positive rate is $\sim 90 \%$ for Q1a(OFF1)-Q1a(OFF2) and $\sim 100 \%$ for $\mathrm{Q} 1 \mathrm{~b}(\mathrm{OFF} 1)-\mathrm{Q} 1 \mathrm{~b}(\mathrm{OFF} 2)$, clearly corresponding to $\mathrm{a}$ non-detection.

We examine the dynamic spectrum of the ON-beam and OFF-beams to determine the time-frequency structure of the emission in observation L570725. In Fig. 6, we show the dynamic spectrum of the beams ON (panel a), OFF 2 (panel b), ON - OFF 2 (panel c), OFF 1 - OFF 2 (panel d), ON/OFF 2 (panel e), and OFF 1/OFF 2 (panel f). The processed dynamic spectrum (with an original time and frequency resolution of $1 \mathrm{~s}$ and $45 \mathrm{kHz}$, respectively) has been binned to $6 \mathrm{~s}$ and a boxcar window 3 pixels across $(135 \mathrm{kHz})$ has been applied along the 
(a)

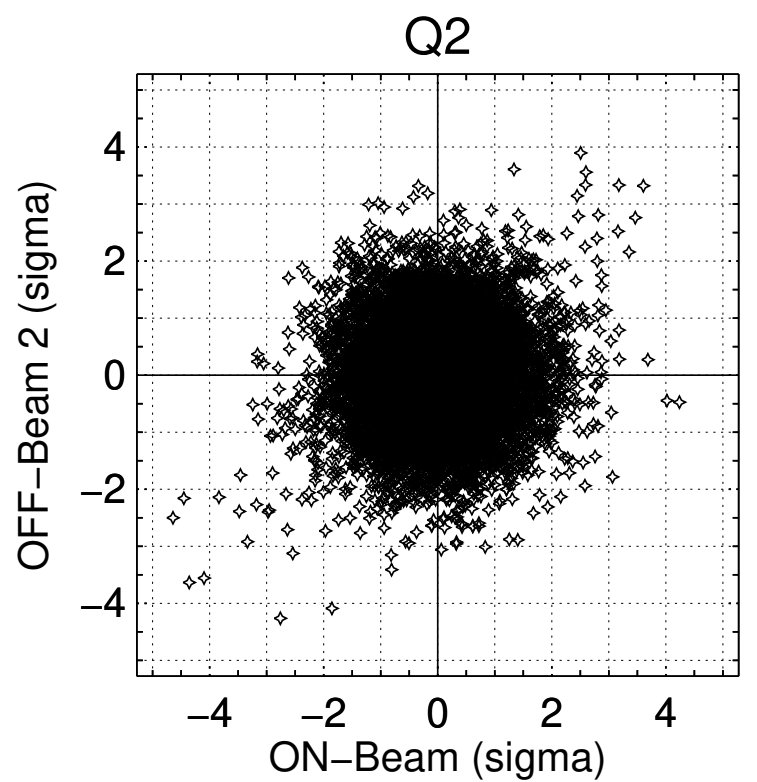

(c)

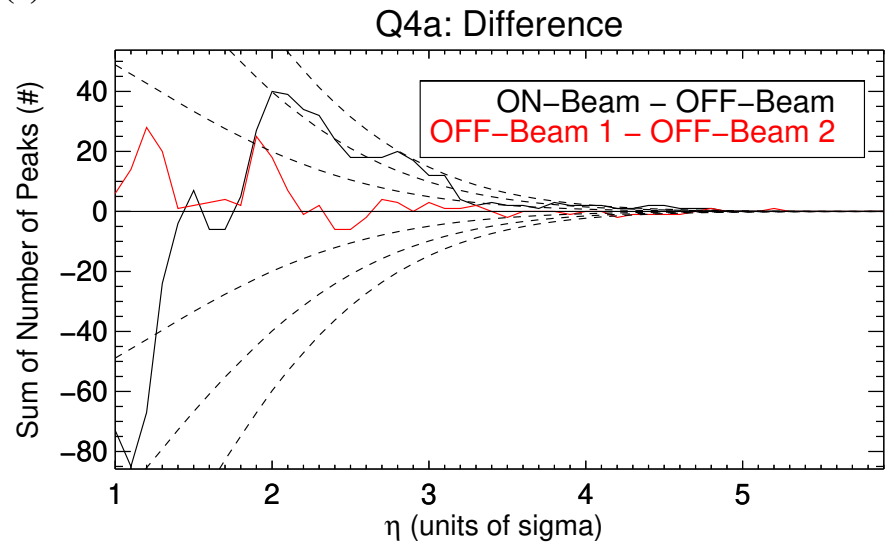

(e)

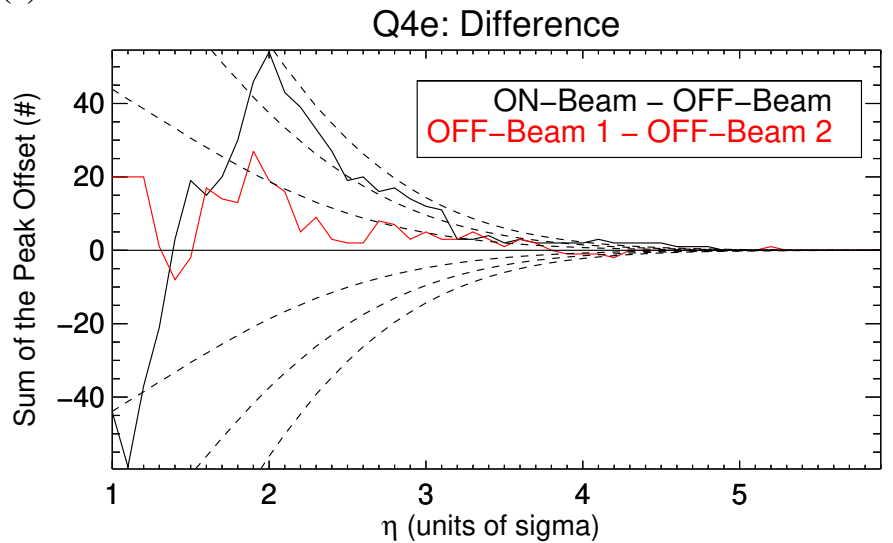

(b)

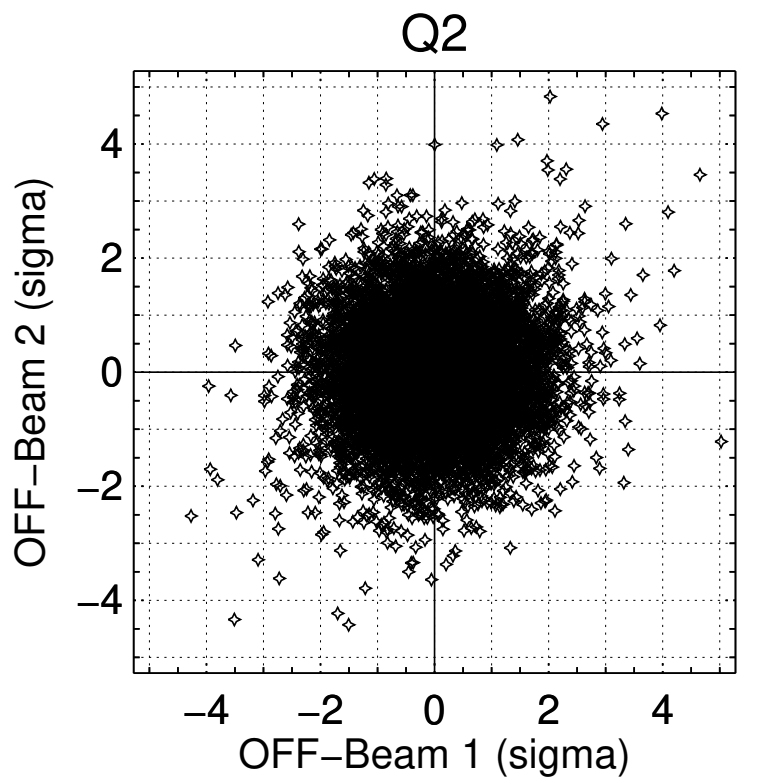

(d)

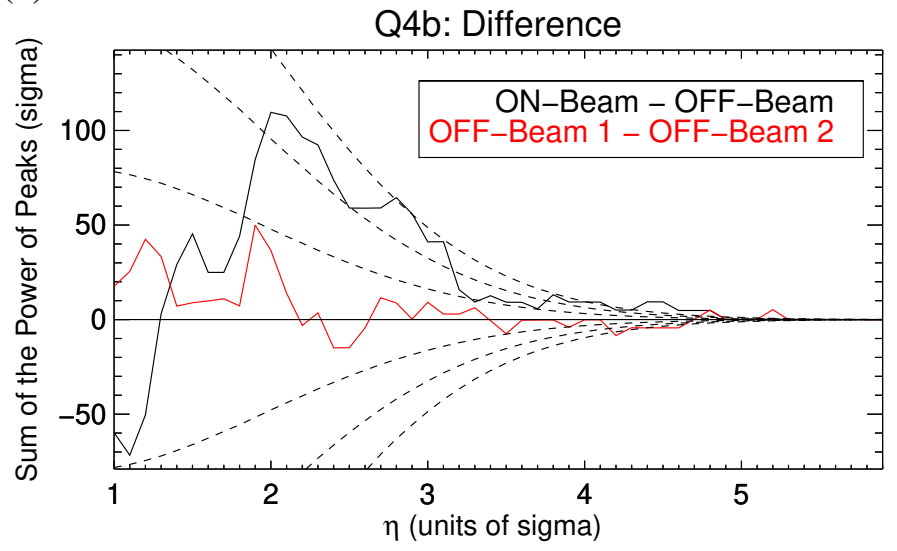

(f)

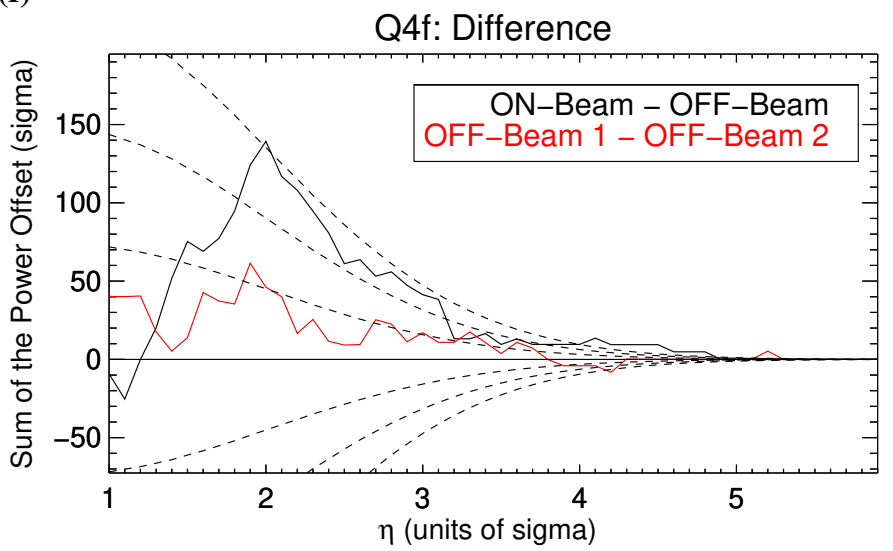

Fig. 4. Q2 (panels $a$ and $b$ ) and beam differences for Q4a (panel c), Q4b (panel d), Q4e (panel e), and Q4f (panel $f$ ) for $\tau$ Boo in observation L569131 in the range 14-38 MHz in Stokes- $V\left(\left|V^{\prime}\right|\right)$. Panel $a$ : Q2 for the ON-beam vs. the OFF beam 2. Panel $b$ : Q2 for the OFF beam 1 vs. the OFF beam 2. Panel c: Q4a (number of peaks). Panel d: Q4b (power of peaks). Panel e: Q4e (peak number offset). Panelf: Q4f (peak power offset). For panels $c-f$ the black lines are the ON-beam difference with the OFF beam 2 and the red lines are the OFF beam difference. The dashed lines are statistical limits $(1,2,3 \sigma)$ of the difference between all the Q4 values derived from 10000 runs using two different Gaussian distributions. In all panels the ON-beam shows an excess above $2 \sigma$ statistical significance and is distinctly different from the OFF-beams. The probability to obtain the OFF beam curve in Q4f (panel $f$ ) by chance is $\sim 81 \%$, whereas it is $7 \times 10^{-4}$ for the ON-beam curve, corresponding to a $3.2 \sigma$ detection. A Kolmogorov-Smirnov statistical test on the two curves for Q4f in panel $f$ conclude that the probability to reject the null hypothesis (that the two curves are drawn from the same parent distribution) is $98 \%$. 
(a)

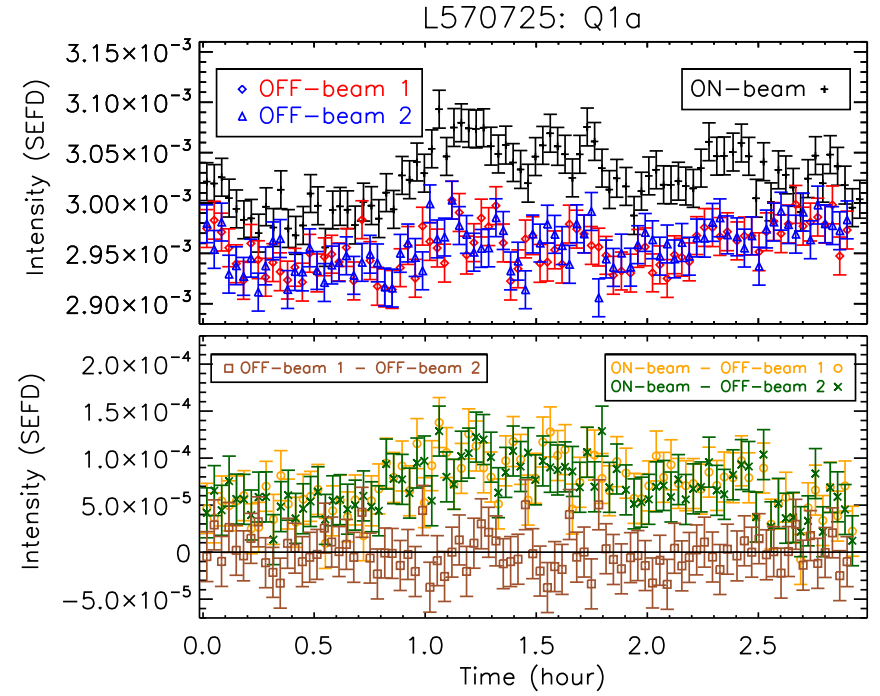

(b)

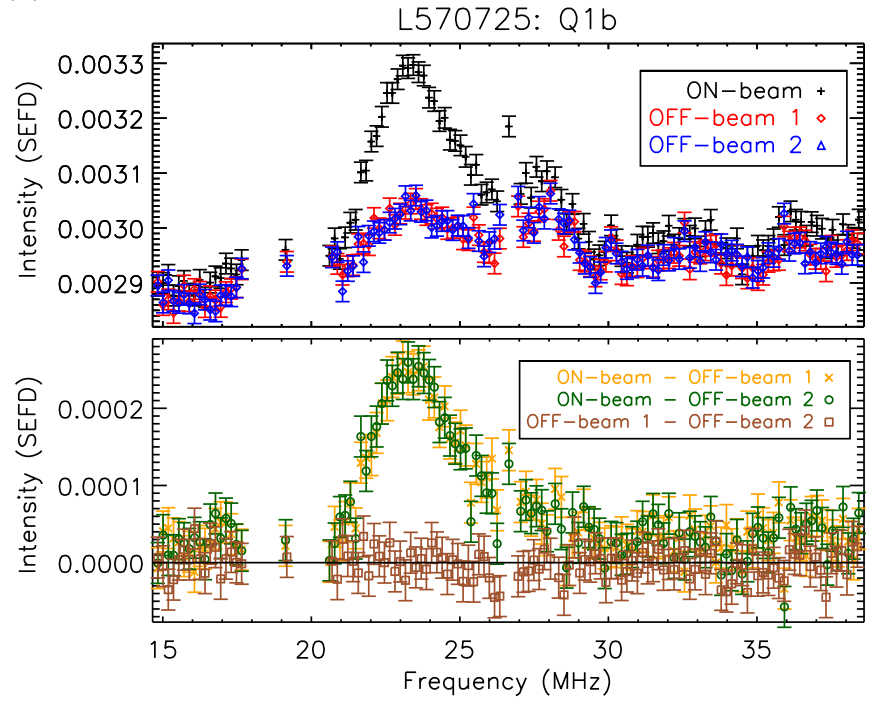

Fig. 5. Time-series (Q1a; panel $a)$ and integrated spectrum (Q1b; panel $b$ ) in Stokes- $V$ (using $\left|V^{\prime}\right|$ as defined in Eqs. (9)-(11) of T19) for $\tau$ Boo during the observation L570725. Panel $a$ : the ON-beam shows excess signal above both OFF beams at all times. Panel $b$ : the signal in the ON-beam is concentrated between 21 and $30 \mathrm{MHz}$ and is distinctly different than in both OFF-beams. In both panels, the two OFF beams are equivalent within the error bars, calculated assuming pure Gaussian noise $(\sigma=1 / \sqrt{b \tau})$. The dynamic spectrum for this signal can be found in Fig. 6. We find by performing Gaussian simulations that the probability to randomly reproduce the signal in the ON-beam curve in Q1a is $2.1 \times 10^{-12}$ and $1 \times 10^{-18}$ for $\mathrm{Q} 1 \mathrm{~b}$. This probability corresponds to a statistically significant detection of $6.9 \sigma$ and $8.6 \sigma$, respectively.

frequency direction to reduce the noise. The ON-beam signal is clearly seen as structured excess emission in panels a and c. These structured features are very similar to the observed radio dynamic spectrum of Jupiter (e.g., Zarka 1998; Marques et al. 2017). There is no equivalent large scale structure in the OFF beam difference plot (panel d), confirming that these two beams are very similar to each other.

Panel $b$ in Fig. 6 shows that the OFF beams contain a replica of the ON signal. The signal is $\sim 1.6$ times fainter in the OFF beam dynamic spectrum when compared to the ONbeam (panel e). We found convincing evidence that the replica signal in the OFF beam originates from the ON beam. Using the pulsar B0809+74 observation L570723 (taken 15 min before observation L570725), we detect the pulsar in the ON-beam but also in the two OFF beams (Fig. G.2). Additionally, we examine the Jupiter observation L568467 (data taken from T19) and find that the ratio of the ON to OFF beam flux is $S(O N) / S(O F F) \sim$ 1.6 (Fig. 7). The flux density ratios (ON/OFF) for the Jupiter observation is consistent with the value found for the $\tau$ Boo observations. The replica signal is likely caused by imperfect phasing of LOFAR at the epoch of the observations, which leads to strong side lobes within the station beam (personal communication from M. A. Brentjens, ASTRON).

We explored several non-astronomical sources (e.g., instrumental systematics) for the signal in the ON-beam in observation L570725. First, during each of our observations, 1-2 LOFAR stations did not operate optimally (cf. Table A.1, second-to-last column). Close inspection showed that the ON-beam signal in this observation was not caused by this (see Appendix $\mathrm{H}$ for details). Next, if the ON beam signal was a permanent instrumental effect, it should also appear in the observation of the pulsar B0809+74 (observation L570723, preceding the $\tau$ Boo observation by $16 \mathrm{~min})$. We find no such large scale features in the range $20-30 \mathrm{MHz}$ in that observation (Fig. G.2). This suggests that the ON-beam signal is either real excess flux in that beam or a time-variable instrumental effect. Finally, low-level features in the integrated spectrum (Q1b) are seen for all ON and OFF beams for all targets (Fig. D.1). For each observation except L570725 these features do not change in time and are similar for the $\mathrm{ON}$ and OFF beam within the error bars. The ONbeam feature in observation L570725 (Fig. D.1f, orange line) has an amplitude comparable to the features seen in ON and OFF beams in all other $\tau$ Boo observations (Figs. D.1e,f). However, both OFF-beams in observation L570725 have a lower amplitude, leading to the detection of an excess signal (ON-OFF). The fact that the ON-OFF excess is caused by a lower level in the OFF beam rather than a higher level in the ON-beam casts some doubt about the astrophysical origin of the detected signal. However, the time-frequency structure in the dynamic spectrum of observation L570725 is not the same as for the other observations. The differences between the dynamic spectra (Fig. I.1) show large scale systematics, which suggests a different source for the emission in this observation.

Summarizing the detailed arguments presented above and in the Appendices, we could not identify an instrumental origin or systematic error for the excess ON-beam signal detected in observation L570725. The amplitudes of ON and OFF signal on that day compared to the other dates encourage us to skepticism, but the time-frequency structure of the signal leaves some room for an astrophysical origin. In all cases, follow-up observations with several radio telescopes are necessary to confirm or invalidate this potential signal.

\section{2. $v$ Andromedae}

For $v$ And, we detected burst emission in the frequency band 14-38 MHz in observation L545197 (2016-09-08) using the Q4f observable (Fig. J.1). The Q4f signal consists of 8 data-points greater than $\eta=2 \sigma$ and a $\mathbf{Q}_{\text {Diff }}$ curve above the 2 sigma Gaussian reference curve (Fig. J.1c).

Similar to Sect. 5.1.1, we find an approximate emission flux of $1.3 \times \sigma_{\text {LOFAR }}(1+0.20) \sim 540_{-240}^{+460} \mathrm{mJy}$ using Eq. (4) and taking into account the $20 \%$ extra noise mentioned in Sect. 3 . The probability that the ON-OFF Q4f curve is a false positive is $1.3 \%$ (assuming Gaussian statistics), equivalent to a $2.2 \sigma$ detection. 
(a)

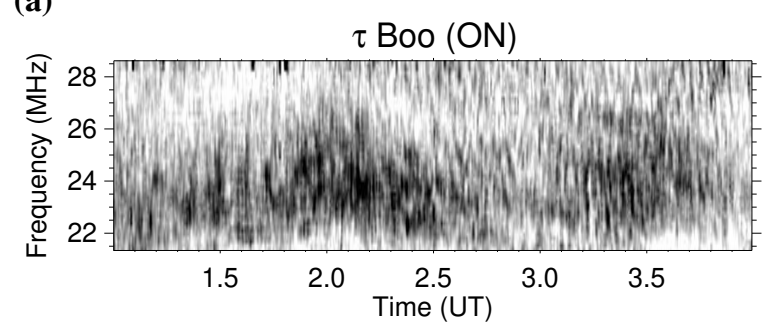

(c)

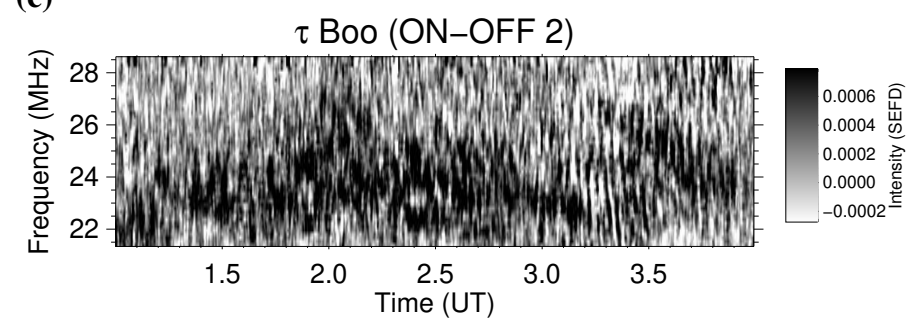

(e)

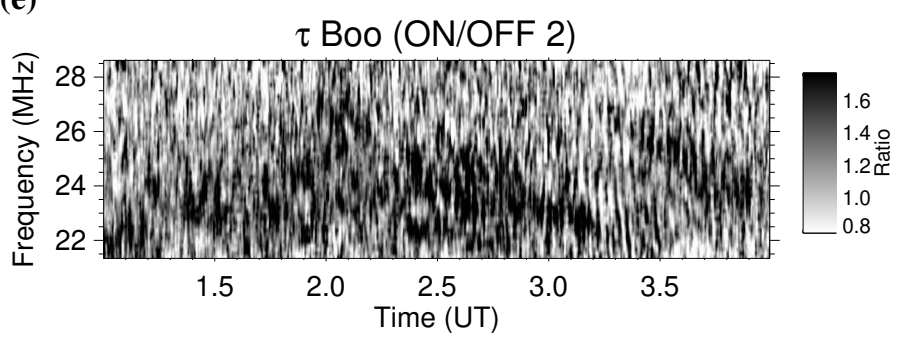

(b)

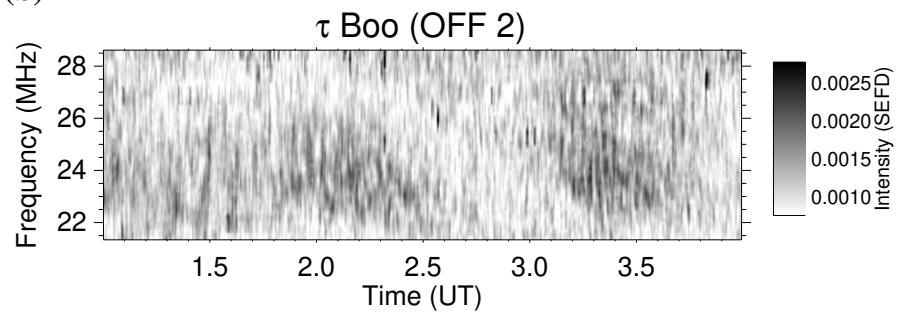

(d)

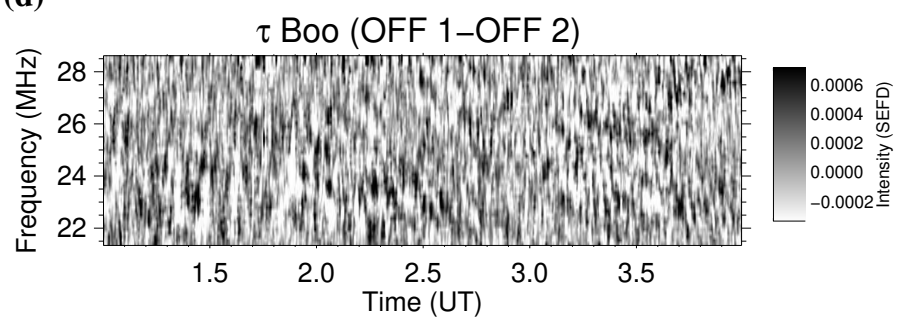

(f)

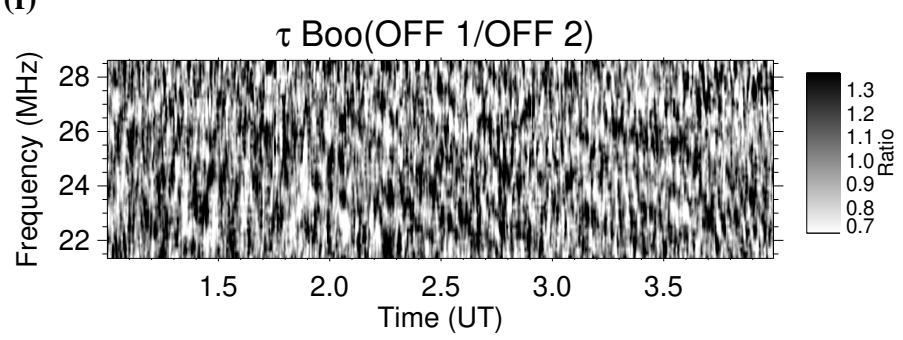

Fig. 6. Dynamic spectra of the $\tau$ Boo b observation L570725 in Stokes- $V\left(\left|V^{\prime}\right|\right)$. Panel a: ON-beam. Panel b: OFF-beam 2. Panel $c$ : ON-beam minus OFF-beam 2. Panel $d$ : OFF-beam 1 minus OFF-beam 2. Panel $e$ : ON-beam divided by the OFF-beam 2. Panel $f$ : OFF-beam 1 divided by the OFF-beam 2. Data from the range $21-29 \mathrm{MHz}$ is shown. The ON-beam signal is clearly seen as structured excess emission in panels $a, c$, and $e$. A faint signal is seen in the OFF beams (e.g., panel c) likely due to ionospheric refraction of the ON-beam signal (also see Appendix G). However, there is no visible excess difference when the two OFF-beams are subtracted by each other (panel $d$ ) suggesting that whatever is causing the faint OFF-beam signal is the same for both beams.

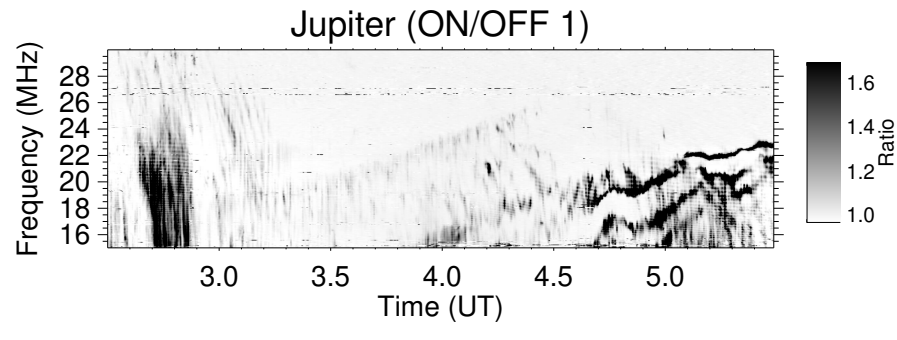

Fig. 7. Dynamic spectrum of the Jupiter observation L568467 (Turner et al. 2019) in Stokes- $V\left(\left|V^{\prime}\right|\right)$. Shown: ON-beam divided by OFFbeam 1 .

By contrast, the probability of obtaining the OFF1-OFF2 curve by chance is $41 \%$. Additionally, we used the K-S statistical test to assess whether the ON-OFF and OFF1-OFF Q4f curves in Fig. J.1f could have been drawn from the same distribution. We find that the probability to reject the null hypothesis (that the two curves are drawn from the same parent distribution) is marginal with a value of $76 \%$. The signal we find for $v$ And is not highly statistically significant, so the possibility for a falsepositive is high. Observations (L545197) also marginally stands out when compared to other observations (detection criterion N6, Fig. C.3). In rare cases (1\%), OFF-beams reach comparable or higher values, adding to the uncertainty on the detection. We present this marginal detection for reference in hope to facilitate and guide future observations of this system.

\section{Discussion}

\subsection{Origin of the detected signals}

We first examine arguments for and against a celestial origin of the signals in the $\tau$ Boo observations (Sect. 6.1.1 for observations L569131, Sect. 6.1.2 for observation L570725). Then, assuming that the bursty emission (L569131) and the slowly varying signal (L570725) are of celestial nature, we explore in greater detail whether those signals could originate from the $\tau$ Boo system (Sect. 6.1.3) and from the exoplanet $\tau$ Boo b itself (Sect. 6.1.4).

\subsubsection{Arguments on whether the detected signal in observation L569131 is of a celestial origin}

The arguments for a celestial origin of the bursty signal in observation L569131 of $\tau$ Boo (i.e. it not being an instrumental effect or RFI) are the following:

1. We find a signal in $\mathrm{Q} 4 \mathrm{f}$ equivalent to a $3.2 \sigma$ detection (Fig. 4). The ON-OFF difference (black curve) is distinctly different from the OFF1-OFF2 difference (red curve red). The K-S test finds that the probability to reject the null hypothesis (that the two curves are drawn from the same parent distribution) is $98 \%$.

2. The signal is circularly polarized, as expected for CMI emission.

3. There is no evidence for low-level residual RFI. Indeed, residual RFI would cause flux to appear simultaneously in the ON- and OFF-beams, and would be seen as points with high 
values close to the main diagonal in the Q2 plot (Fig. 4a). Also, the main diagonal is excluded in the calculation of the $\mathrm{Q} 4 \mathrm{f}$ observable quantity.

An argument against a celestial origin is:

1. There is a possibility that low-level RFI could cause the signal in the event that the RFI conditions between the ON and OFF beams are significantly different. However, there is no evidence that the RFI is different between the beams. Also, we have demonstrated that the RFI mitigation step in the pipeline is efficient (Turner et al. 2017a, 2019).

Since we do not find potential false positives for the bursty signal, this is likely a real detection of celestial emission and not an instrumental effect. However, a detection at $3.2 \sigma$ level is not highly significant and calls for confirmation via follow-up observations.

\subsubsection{Arguments on whether the detected signal in observation L570725 is of a celestial origin}

Likewise, we now summarize the arguments for and against the possibility that the slowly varying signal in observation L570725 of $\tau$ Boo is indeed real celestial emission. The arguments for a real detection are:

1. There is a clear signal in Q1a equivalent to $6.9 \sigma$ and Q1b equivalent to $8.5 \sigma$ (Fig. 5).

2. The signal is found to be circularly polarized, as expected for CMI emission.

3. The signal has a complex structure in time-frequency (Fig. 6) reminiscent of Jupiter radio arcs (e.g., Marques et al. 2017).

4. The structures seen in the dynamic spectra of the ONbeam are distinctly different than all OFF-beams for every $\tau$ Boo observation (Fig. I.1).

5. All observations in this study were processed identically with the BOREALIS pipeline (Vasylieva 2015; Turner et al. 2017a; T19). The L570725 signal is the only one among many observations and runs with similar observing conditions (Table A.1).

6. All OFF-beams in each individual observation are consistent with each other within expected Gaussian error bars (Fig. D.1). There is no indication that any of the beams (ON or OFF) in any observation has abnormal behavior.

7. All ON and OFF beams in each individual observation except L570725 are also consistent with each other assuming Gaussian error bars (Fig. D.1).

8. The RFI mitigation in the pipeline is efficient (Turner et al. 2017a, 2019) and there is no evidence that RFI remains in the data. Also, the signal does not look like RFI.

9. The ON and OFF beams are close enough to each other (Table A.2; $2-3^{\circ}$ ) so that the ionospheric fluctuations should be quite similar in all three beams.

The arguments against a celestial signal and potential explanations are as follows:

1. In observation L570725, the two OFF-beams contain weak replicas of the ON beam signal (Fig. 6). We believe this replica signal can be explained by imperfect phasing of the LOFAR core. This assessment is based on the analysis of observations of Jupiter (Fig. 7) and the pulsar B0809+74 (Fig. G.1), taken in the same mode as our exoplanet observations.

2. During each of our observations, 1-2 LOFAR stations did not operate optimally and showed some spurious behaviour in the frequency range $21-30 \mathrm{MHz}$ (Fig. H.1). A quantitative analysis suggests that this is not the origin of the apparent signal (Fig. H.1).
3. The ON-beam excess in observation L570725 is caused by an unusually low signal in both OFF-beams rather than an unusually high high signal in the ON-beam. Indeed, the ONbeam feature at $21-30 \mathrm{MHz}$ has an amplitude comparable to the features seen in ON and OFF beams in all other $\tau$ Boo observations (Fig. D.1e). However, the structure in the dynamic spectrum of the observation L570725 is not the same as in all other observations, especially those with large-scale systematics (Fig. I.1). It is unclear why observation L570725 is different from our other $\tau$ Boo observations. We cannot rule out a small temporary variation within the telescope.

We do have some doubt about the slowly varying signal. Further observations may show whether such a signal is confirmed; if not, it may have been caused by an instrumental artifact.

\subsubsection{Arguments for the detected signals originating from the $\tau$ Boo system}

Assuming the bursty and slowly variable signal are real and of celestial origin, do these signals originate from the $\tau$ Boo system? Or could other possible radio sources be at the origin of the detected circularly polarized signals?

In general, most astrophysical radio sources display little circular polarization. There is only one known object detected in Stokes- $I$ at $150 \mathrm{MHz}$ in the TGSS survey (Intema et al. 2017) within the 13.8 arcmin $\tau$ Boo ON-beam of the LOFAR core. This same object is also detected in the $1.4 \mathrm{GHz}$ VLA FIRST Survey Catalog (Helfand et al. 2015). This object is probably a background radio-loud quasar since it produces emission over a large frequency range. This quasar is likely not the origin of our detected signals since quasars generally have a small degree of circular polarization ( $<2 \%$, e.g., Bower et al. 2002). During all our observations, simultaneous observations of Jupiter between 10 and $40 \mathrm{MHz}$ were taken using the Nançay Decameter Array (NDA; Boischot et al. 1980; Lamy et al. 2017). No emission was seen during the time period of the observation L570725. Therefore, it is very unlikely that the ON-beam signal is caused by Jupiter decameter emission in a side lobe. Radio signals from artificial satellites are also sometimes polarized. However, the detected signals are not from a low Earth orbit (LEO) spacecraft because a LEO satellite would pass from horizon to horizon in several minutes; in our data, the detected signals are visible for much longer timescales. It is also unlikely that the signal is a geostationary (GEO) spacecraft since all three beams are close together on the sky and the same beams were used for all observations of each planet (cf. Table A.2). Besides, the timefrequency structure of the signal does not resemble a satellite beacon. As we do not see any other compelling explanation, we conclude it is indeed likely that the source of the detected signals is located within the $\tau$ Boo system.

\subsubsection{Arguments for the detected signals originating from the exoplanet $\tau$ Boo b}

Several physical arguments suggest that the detected signals originate from the planet $\tau$ Boo b rather than its host star. The planetary emission is expected to be much stronger than the stellar emission for hot Jupiters (e.g., Grießmeier et al. 2005b; Zarka et al. 1997; Zarka 2011). Grießmeier et al. (2005b) estimated that the radio emission from the planet $\tau$ Boo b would be stronger by several orders of magnitude than the galactic background, the quiet and quiescent stellar emission, and stellar noise storms. While stellar radio bursts can in principle be as intense as the planetary emission, there is no evidence that $\tau$ Boo undergoes 
large flare events; no such flares have been seen in two years of long-term monitoring of the system with the MOST satellite (Walker et al. 2008) and X-ray observations from XMM-Newton (Mittag et al. 2017).

The Radio-Magnetic Bode's law (Zarka et al. 2001, 2018; Zarka 2007, 2018; Grießmeier et al. 2007a) predicts that for close-in planets an emission flux up to $10^{6-7}$ times Jupiter's radio flux should be possible. Zarka (2010) tested this scaling law by examining the radio emission from magnetized binary stars. They found that the radio emission (likely not due to CMI) from the RS CVn stellar system V711 Tauri matched approximately the extrapolation of the law derived for the Solar System (Mottez \& Zarka 2014). Their result suggests that the radio-Magnetic Bode's law could hold for 10 orders of magnitude above the range of solar system planets. The recent detection of Ganymede-induced radio emission confirms the Radio-Magnetic Bode's law (Zarka et al. 2018). Several models suggest that there could be deviations of one order of magnitude from this scaling law (Nichols 2011; Saur et al. 2013; Nichols \& Milan 2016), but the deviation remains modest compared to the large-scale tendency.

Stellar and planetary radio emissions are also expected to have different polarization properties (Zarka 1998; Grießmeier et al. 2005b). The detected signal is circularly polarized, which is expected for planetary CMI emission. Quiet and quiescent stellar radio emission usually have a low polarization degree (quiescent emission of $\mathrm{M}$ dwarfs has occasionally been observed to reach a polarization degree of 50\%, Güdel 2002, and references therein). Stellar radio bursts can be circularly polarized if they are caused by the CMI. However, there is no previous evidence of radio flares from the $\tau$ Boo system. More importantly, a star needs to be strongly magnetized $\left(\mathrm{B}_{\text {star }}>10-100 \mathrm{G}\right)$ to produce CMI emission that is not quenched by the coronal plasma (Zarka 2007). For $\tau$ Boo, the mean stellar magnetic field has been measured to be 1.7-3.9 G (Catala et al. 2007; Donati et al. 2008; Fares et al. 2009, 2013; Mengel et al. 2016; Jeffers et al. 2018). CMI emission from the star over the frequency range of the detected signals is thus unlikely. Still, since the surface magnetic field maps (e.g., Vidotto et al. 2012) do not cover small-scale magnetic structures (Mittag et al. 2017), CMI emission from small active regions cannot be not ruled out. Also, the magnetic field of the M-dwarf companion star $\tau$ Boo B is unknown. For this reason, stellar flares cannot be ruled out and could potentially be the cause for the detected radio signal.

CMI emission from the planet $\tau$ Boo $b$ remains a possible cause for the detected circularly polarized radio signal. It it not the only possible source, the other being radio emission by stellar flares. A major argument in favor of planetary radio emission would be the detection of a radio signal compatible with the planetary rotation period. Follow-up observations are required to confirm the presence of this faint signal, and subsequently verify its origin.

\subsection{Physical constraints on the planetary systems}

\subsection{1. $\tau$ Boötis}

Assuming that at least one of the two detections (L569131 and L570725) is real and due to CMI emission from the planet (Table 4), we can constrain the maximum surface magnetic field of the planet $\tau$ Boo $\mathrm{b}$ to be in the range $\sim 5-11 \mathrm{G}$. CMI emission is produced at the local gyrofrequency, $v_{g}(\mathrm{MHz})=2.8 \times B_{\mathrm{p}}(G)$, and we use the full frequency range of the detected signals (15$30 \mathrm{MHz}$ ) to constrain the magnetic field range for $\tau$ Boo $b$. This value is slightly smaller than Jupiter's maximum surface polar magnetic field of 14 G (Acuna \& Ness 1976; Connerney 1993). The magnetic moment $\left(\mathcal{M}_{\mathrm{p}}\right)$ of the planet that can be expressed as:

$$
\mathcal{M}_{\mathrm{p}}=\mathcal{M}_{\text {Jup }}\left(\frac{B_{\mathrm{p}}}{B_{\mathrm{Jup}}}\right)\left(\frac{R_{\mathrm{p}}}{R_{\mathrm{Jup}}}\right)^{3},
$$

where $\mathrm{B}_{\mathrm{p}}$ and $\mathrm{B}_{\mathrm{Jup}}$ are the exoplanet's and Jupiter's polar magnetic field strength, and $R_{\mathrm{p}}$ and $\mathrm{R}_{\mathrm{Jup}}$ are the radius of the planet and Jupiter. $R_{\mathrm{p}}$ is estimated to $1.06 \mathrm{R}_{\mathrm{Jup}}$ using a parametric equation for the radius of an irradiated planet (Wang \& Ford 2011). We find a magnetic moment $\mathcal{M}_{\mathrm{p}}$ of $0.94 \mathcal{M}_{\text {Jup }}$ for $\tau$ Boo $\mathrm{b}$. Our tentative $\tau$ Boo detections are labeled as observation 9 in Fig. 1. The figure shows that the magnetic field and emission strengths derived for $\tau$ Boo $\mathrm{b}$ are consistent with the predictions by Grießmeier et al. (2007a) and Grießmeier (2017) (in particular with the NR model). Our derived magnetic field strengths could place constraints on the dynamo theory. The field strength estimated for the planet should allow for a sustained planetary magnetosphere, thus protecting it from the stellar wind (Nicholson et al. 2016). The detection of radio emission from $\tau$ Boo $\mathrm{b}$ is also interesting in view of atmospheric simulations. For a typical hot Jupiter, CMI quenching by a high plasma frequency in the planetary ionosphere can potentially prevent radio emission; for $\tau$ Boo b, however, this problem is alleviated by the high planetary mass and thus low electron density in the ionosphere (Weber et al. 2017a,b, 2018).

We can estimate the power and brightness temperature of the observed emission in both detections (L569131 and L570725). The power of the polarized emission can be estimated by:

$P=S \Omega d^{2} \Delta v$,

where $S$ is the observed polarized flux density, $\Omega$ is the solid angle filled by the CMI emission beam (assumed to be in the range $0.16-1.6$ sr similar to Jupiter's decameter emission; Zarka et al. 2004), $d$ is the distance to the planet (15.6 pc for $\tau$ Boo b), and $\Delta v$ is the frequency range (Col. 3 of Table 4). Using Eq. (6) and the maximum observed flux, we find a power of $6.3 \times 10^{14}-2.0 \times 10^{16} \mathrm{~W}$ for $\tau$ Boo b (Table 4). This derived power is $10^{4-5}$ greater than Jupiter's maximum decametric emission $\left(4.5 \times 10^{11} \mathrm{~W}\right.$; Zarka et al. 2004). The derived power is consistent with theoretical predictions (Grießmeier et al. 2007a, Grießmeier 2017) and compatible with our tests on LOFAR beam-formed data (T19) which showed that such a large power is needed to be detectable from $\sim 15 \mathrm{pc}$ distance. The brightness temperature $\left(\mathrm{T}_{\mathrm{B}}\right)$ of the radio source is:

$T_{\mathrm{B}}=\frac{S}{\omega k} \lambda^{2}$

where $\omega$ is the angular size of the emission source, $\mathrm{k}$ is the Boltzmann constant, and $\lambda$ is the wavelength. With the power of the exoplanetary radio emission being much higher than that of Jupiter, Eq. (7) means that either the brightness temperature of the emission from $\tau$ Boo $b$ is much higher than that of Jupiter, or the source region is much more extended. A source size of $1 R_{\text {Jup }}$ implies a brightness temperature of $4.2 \times 10^{17}$ to $2.0 \times 10^{18} \mathrm{~K}$ (Table 4), similar to the brightness temperature of Jovian radio bursts $\left(2 \times 10^{17} \mathrm{~K}\right.$; Zarka 1992), but the latter is reached in sources of size 10-100 km only (Zarka et al. 1996). These flux densities require a very high Poynting flux due to the proximity of the planet to the star, following the radio magnetic scaling law (e.g., Zarka et al. 2001, 2018; Grießmeier et al. 2007a). Once 
the planetary origin of the signal is established, modeling of the dynamic spectrum with a software like ExPRES (Hess \& Zarka 2011; Louis et al. 2019) will provide more information about the emission source and magnetic field structure.

\subsection{2. $v$ Andromedae}

The only radio signal tentatively seen from the $v$ And system is at $2.2 \sigma$ level in observation L54519. If this marginal detection is real, the flux from the system is $540_{-240}^{+460} \mathrm{mJy}$. If it is a falsepositive, we can derive a $3 \sigma$ upper limit of $124 \mathrm{mJy}$ from the range 26-73 MHz using the Q1a observable for slowly varying emission. For the moment, we classify this as a non-detection, implying that either the observations were not sensitive enough, the planetary magnetic field is too weak to emit at the observed frequencies, or that Earth was outside the beaming pattern of the radio emission at the time the observations were carried out.

\subsubsection{Cancri}

We can estimate an upper limit on the radio emission from our non-detection of the $55 \mathrm{Cnc}$ system. We find a $3 \sigma$ upper limit of $73 \mathrm{mJy}$ from the range $26-73 \mathrm{MHz}$ using the Q1a observable for slowly varying emission. Using the attenuated Jupiter modeling done in T19, this is equivalent to a flux density less than $10^{5}$ times the peak flux of Jupiter's decametric burst emission $\left(\sim 5 \times 10^{6} \mathrm{Jy}\right.$; Zarka et al. 2004). Due to our full orbital coverage of $55 \mathrm{Cnc}$, we can rule out beaming effects (Earth outside the beaming pattern) as the cause of our non-detection. Therefore, our non-detection of $55 \mathrm{Cnc}$ implies that either the planetary magnetic field is too weak to emit at the observed frequencies or that the emission is too weak.

\subsection{Limitations of beamformed observations}

Beamformed observations provide very good resolution in time and frequency (in the present study, we used $10 \mathrm{~ms}$ and $3 \mathrm{kHz}$ ). They are extremely powerful for studying strong (e.g., Jupiter; Marques et al. 2017, the Sun; Pick \& Vilmer 2008), periodic (e.g., pulsars; Pilia et al. 2016), or dispersed bursts (e.g., RRATs; Karako-Argaman et al. 2015, FRBs; CHIME/FRB Collaboration 2019), as well as spectral lines (e.g., RRLs; Asgekar et al. 2013). The situation is much less favorable for weak non-periodic broadband bursts without a clear dispersion signature such as radio bursts expected from exoplanets (e.g., Zarka et al. 1997). This emission is the most difficult kind of emission to detect at low-frequencies in beamformed mode.

Imaging observations excel at detecting continuous and moderately bursty signals. Progress in sky imaging at lowfrequencies has been huge recently (e.g., LoTSS, Shimwell et al. 2019). For low-frequency radio telescopes like LOFAR-LBA (van Haarlem et al. 2013) and NenuFAR (Zarka et al. 2012, 2020) imaging is still difficult, computationally expensive, limited by RFI and ionospheric effects, and sometimes impossible due to the lack of good calibrators, thus beamformed observations are still relevant. There has been recent development of a software package called DynSpecMS ${ }^{5}$ (Tasse et al., in prep.) that produces low resolution ( $\sim 8 \mathrm{~s} \times \sim 10 \mathrm{kHz}$ in LoTSS) dynamic spectra from the calibrated visibilities of imaging data. DynSpecMS will provide as many OFF-beams as image pixels, and thus may be a good alternative to beamformed observations when high-quality imaging is possible (e.g., LOFAR-HBA).

https://github.com/cyriltasse/DynSpecMS
Beamformed observations retain several advantages over imaging data. They have higher time resolution (in our case $10 \mathrm{msec}$, compared to several seconds), which can be used to mitigate RFI on shorter time scales, although not on individual station level. Beamformed observations also excel at the detection of short bursty signals. The computational cost of their processing is significantly less than for imaging observations since only a handful of pixels have to be analyzed. As done in this paper, a typical observation in beamformed mode should involve 1 ON-beam and 2 to 3 simultaneous OFF-beams (e.g. Zarka et al. 1997; Turner et al. 2017a).

However, beamformed observations also suffer from several drawbacks. Any spurious emission in the side lobes or from instrumental origin is difficult to distinguish from real emission. Having several simultaneous OFF beams is absolutely crucial in that case. We found that two OFF beams are a critical minimum to be able to compare statistics as done in this paper. Future observations may need to have more OFF beams ( 3 or 4 surrounding the target), even at the expense of the frequency bandwidth (or increasing the data volume in the case of the LOFAR core), to better evaluate the background and side lobe effects. Also, if a variable strong low-frequency source is in the sky (e.g., Jupiter or the Sun), it should be monitored (e.g., with a dedicated beam) in order to identify any emission that it could contribute to the ON-beam.

It is currently not possible to conclude whether the imaging or beamformed approach is better adapted for the study of exoplanetary radio emission. As long as this question is unanswered, both types of observations should be pursued. In the ideal case, observations should be executed in both modes simultaneously whenever possible. This paper has demonstrated that beamformed observations can provide important information; as a consequence, they will certainly continue to play a useful role in studying exoplanetary radio emission.

\section{Conclusions}

In this study, we obtained and analyzed LOFAR-LBA beamformed circularly polarized (Stokes- $V$ ) observations of the exoplanetary systems 55 Cancri, $v$ Andromedae, and $\tau$ Boötis. For the $\tau$ Boo system, we tentatively detect circularly polarized burst emission in the range $14-21 \mathrm{MHz}$ with a statistical significance of $3.2 \sigma$ (Table 4; Fig. 4). We cannot rule out stellar flares as the source of the emission and emission from the planet $\tau$ Boo remains a possible cause (see Sect. 6.1.4); follow-up observations are required (see next Section). For $\tau$ Boo, we also detect slowly variable emission in the range $21-30 \mathrm{MHz}$ with a significance of $8.6 \sigma$ (Table 4; Fig. 5). A thorough analysis did not allow any firm conclusion: the existence of this signal can neither be confirmed with certainty, nor can it fully be refuted (Sect. 6.1.2). Our observations of the $55 \mathrm{Cnc}$ system cover twice the full orbit of the inner planet (Fig. 2b). This is the first time an exoplanetary radio search project has full orbital coverage of an exoplanetary target. No emission is seen from $55 \mathrm{Cnc}$ and we placed a $3 \sigma$ upper limit of $73 \mathrm{mJy}$ on the system from our observations. For the $v$ And system, we found burst emission in the range $14-38 \mathrm{MHz}$ with a marginal statistical significance of $2.2 \sigma$. We classify this as a non-detection. For $\tau$ Boo $\mathrm{b}$ and $v$ And $\mathrm{b}$ the phase coverage is $25 \%$ (Fig. 2c) and 40\% (Fig. 2d), respectively. For this reason, we cannot rule out that we have missed radio emission concentrated at specific orbital phases not covered.

Using the $\tau$ Boo detections (L569131 and L570725) from the range $15-30 \mathrm{MHz}$ and assuming the emission is from the planet 
and generated by the CMI mechanism (Sect. 6), we derived a maximum surface polar magnetic field for $\tau$ Boo b between $\sim 5-11 \mathrm{G}$. The signals for $\tau$ Boo b range from 190 to $890 \mathrm{mJy}$ with an emitted power of $6.3 \times 10^{14}-2.0 \times 10^{16} \mathrm{~W}$ and a brightness temperature of $0.42-2.0 \times 10^{18} \mathrm{~K}$ (Table 4). The magnetic field and emission strengths derived for $\tau$ Boo $\mathrm{b}$ are consistent with the predictions (Table 1; Fig. 1) by Grießmeier et al. (2007a) and Grießmeier (2017).

Follow-up low-frequency radio observations (e.g., LOFAR, UTR-2, LWA-OLWA, NenuFAR) are needed to confirm our tentative detections from $\tau$ Boo and the marginal detection from $v$ And. Searching for periodicity in the detected signals will be crucial in confirming their origin and nature. Simultaneous observations between two facilities (e.g LOFAR and NenuFAR) is highly encouraged to rule out possible false-positives due to instrumental effects. We also hope to incorporate machine learning techniques (e.g., Baron 2019) into BOREALIS in the future to more efficiently search through the post-processing outputs.

Ancillary data on these targets are also critical. The planetary mass and planetary inclination are well constrained from high-resolution spectroscopy observations (Brogi et al. 2012; Rodler et al. 2012; Piskorz et al. 2017). The planetary rotation period could also be constrained using high-resolution data (e.g., Brogi et al. 2016). Continued or simultaneous monitoring of stellar lightcurves for stellar flares can be done with the northern version of Evryscope that is currently undergoing commission (Law et al. 2015; Ratzloff et al. 2019; Howard et al. 2019). X-ray monitoring is also useful as an indicator to discriminate the planetary signal from that of the star. Follow-up and if possible simultaneous stellar magnetic field maps (e.g., obtained by Zeeman-Doppler Imaging) and wind measurements (e.g., astrospheric absorption) would also be extremely beneficial for the interpretation of future observations.

Beamformed observations are notoriously difficult to exploit for exoplanetary radio emission. Despite these drawbacks, they can provide useful information, as demonstrated in this paper. We expect them to continue to play an important role in the future alongside other observing modes such as imaging observations.

Acknowledgements. J.D.T was partially funded by the National Science Foundation Graduate Research Fellowship under Grant No. DGE-1315231. J.D.T was also funded by a postdoctoral research position at Cornell University. Most of the work on this paper was done during J.D.T.s $\mathrm{PhD}$ at the University of Virginia under the supervision of Robert E. Johnson. Part of this research was carried out at the Jet Propulsion Laboratory, California Institute of Technology, under a contract with the National Aeronautics and Space Administration. This work was supported by the "Programme National de Planétologie" (PNP) of CNRS/INSU co-funded by CNES and by the "Programme National de Physique Stellaire" (PNPS) of CNRS/INSU co-funded by CEA and CNES. This research has made use of the Extrasolar Planet Encyclopaedia (exoplanet.eu) maintained by J. Schneider (Schneider et al. 2011), the NASA Exoplanet Archive, which is operated by the California Institute of Technology, under contract with the National Aeronautics and Space Administration under the Exoplanet Exploration Program, and NASA's Astrophysics Data System Bibliographic Services This research has also made use of Aladin sky atlas developed at CDS, Strasbourg Observatory, France (Bonnarel et al. 2000; Boch \& Fernique 2014) In this paper, all the physical characteristics for the pulsar B0809+74 were taken from the ATNF Pulsar Catalogue (Manchester et al. 2005) located at http://www.atnf.csiro.au/research/pulsar/psrcat. This paper is based on data obtained with the International LOFAR Telescope (ILT) under project codes LC2_018, LC5_DDT_002, LC6_010, and LC7_013. LOFAR (van Haarlem et al. 2013) is the Low Frequency Array designed and constructed by ASTRON. It has observing, data processing, and data storage facilities in several countries, that are owned by various parties (each with their own funding sources), and that are collectively operated by the ILT foundation under a joint scientific policy. The ILT resources have benefited from the following recent major funding sources: CNRS-INSU, Observatoire de Paris and Université d'Orléans, France; BMBF, MIWF-NRW, MPG, Germany; Science Foundation
Ireland (SFI), Department of Business, Enterprise and Innovation (DBEI), Ireland; NWO, The Netherlands; The Science and Technology Facilities Council, UK. We acknowledge the use of the Nançay Data Center computing facility (CDN - Centre de Données de Nançay). The CDN is hosted by the Station de Radioastronomie de Nançay in partnership with Observatoire de Paris, Université d'Orléans, OSUC and the CNRS. The CDN is supported by the Region Centre Val de Loire, département du Cher. We thank the ASTRON staff for their help with these observations (pre- and post observing) and understanding where the systematics originate. We also thank the following people who were co-I's on the LOFAR observing proposals (LC2_018, LC5_DDT_002, LC6_010, and LC7_013) and helped us obtain the data presented in this paper: Alain Lecavelier des Etangs, Alexander Konovalenko, Artie Hatzes, Cyril Tasse, Gregg Hallinan, Iaroslavna Vasylieva, Robert E. Johnson, Sander ter Veen, Vladimir Ryabov, and Walid Majid. This paper uses data from the TGSS survey (Intema et al. 2017) and we thank the staff of the GMRT that made this survey possible. GMRT is run by the National Centre for Radio Astrophysics of the Tata Institute of Fundamental Research. NDA observations of Jupiter were used to monitor its emission. The NDA is hosted by the Nançay Radio Observatory/ Unité Scientique de Nançay of the Observatoire de Paris (USR 704-CNRS, supported by Université d'Orléans, OSUC, and Region Centre in France). We thank the anonymous referee for their useful and thoughtful comments. Facilities: LOFAR (van Haarlem et al. 2013) Software: BOREALIS (Vasylieva 2015; Turner et al. 2017a, 2019); IDL Astronomy Users Library (Landsman 1995); Coyote IDL created by David Fanning and now maintained by Paulo Penteado (JPL).

\section{References}

Acuna, M. H., \& Ness, N. F. 1976, J. Geophys. Res., 81, 2917

Alexander, R. D., Wynn, G. A., Mohammed, H., Nichols, J. D., \& Ercolano, B. 2016, MNRAS, 456, 2766

Asgekar, A., Oonk, J. B. R., Yatawatta, S., et al. 2013, A\&A, 551, L11

Baron, D. 2019, ArXiv e-prints [arXiv:1904 . 07248]

Bastian, T. S., Dulk, G. A., \& Leblanc, Y. 2000, ApJ, 545, 1058

Bastian, T. S., Villadsen, J., Maps, A., Hallinan, G., \& Beasley, A. J. 2018, ApJ, 857,133

Bisikalo, D. V., Kaigorodov, P. V., Ionov, D. E., \& Shematovich, V. I. 2013, Astron. Rep., 57, 715

Boch, T., \& Fernique, P. 2014, ASP Conf. Ser., 485, 277

Boischot, A., Rosolen, C., Aubier, M. G., et al. 1980, Icarus, 43, 399 Bonnarel, F., Fernique, P., Bienaymé, O., et al. 2000, A\&AS, 143, 33

Bower, G. C., Falcke, H., \& Mellon, R. R. 2002, ApJ, 578, L103

Bower, G. C., Loinard, L., Dzib, S., et al. 2016, ApJ, 830, 107

Brogi, M., Snellen, I. A. G., de Kok, R. J., et al. 2012, Nature, 486, 502

Brogi, M., de Kok, R. J., Albrecht, S., et al. 2016, ApJ, 817, 106

Burke, B. F., \& Franklin, K. L. 1955, J. Geophys. Res., 60, 213

Butler, R. P., Marcy, G. W., Williams, E., Hauser, H., \& Shirts, P. 1997, ApJ, 474, L115

Catala, C., Donati, J. F., Shkolnik, E., Bohlender, D., \& Alecian, E. 2007, MNRAS, 374, L42

Cauley, P. W., Shkolnik, E. L., Llama, J., \& Lanza, A. F. 2019, Nat. Astron., 408, 143

CHIME/FRB Collaboration (Amiri, M., et al.) 2019, Nature, 566, 230

Christensen, U. R. 2010, Space Sci. Rev., 152, 565

Christensen, U. R., Holzwarth, V., \& Reiners, A. 2009, Nature, 457, 167

Connerney, J. E. P. 1993, J. Geophys. Res., 98, 18659

Cowley, S. W. H., Bunce, E. J., \& Nichols, J. D. 2003, J. Geophys. Res. Space Phys., 108, 8002

Cuntz, M., Saar, S. H., \& Musielak, Z. E. 2000, ApJ, 533, L151

de Gasperin, F., Lazio, T. J. W., \& Knapp, M. 2020, A\&A, 644, A157

de Pater, I. 2000, Perspectives on Radio Astronomy: Science with Large Antenna Arrays, ed. M. P. van Haarlem, 327

Desch, M. D. 1988, Geophys. Res. Lett., 15, 114

Desch, M. D., \& Kaiser, M. L. 1984, Nature, 310, 755

Donati, J.-F., Moutou, C., Farès, R., et al. 2008, MNRAS, 385, 1179

Fares, R., Donati, J. F., Moutou, C., et al. 2009, MNRAS, 398, 1383

Fares, R., Moutou, C., Donati, J. F., et al. 2013, MNRAS, 435, 1451

Farrell, W. M., Desch, M. D., Lazio, T. J., Bastian, T., \& Zarka, P. 2003, ASP Conf. Ser., 294, 151

Farrell, W. M., Desch, M. D., \& Zarka, P. 1999, J. Geophys. Res., 104, 14025

Farrell, W. M., Lazio, T. J. W., Zarka, P., et al. 2004, Planet. Space Sci., 52, 1469

Fischer, D. A. 2018, 55 Cancri (Copernicus): A Multi-planet System with a Hot Super-Earth and a Jupiter Analogue, 2677

Folsom, C. P., Ó Fionnagáin, D., Fossati, L., et al. 2020, A\&A, 633, A48 
Foster, G., Poppenhaeger, K., Alvarado-Gómez, J. D., \& Schmitt, J. H. M. M. 2020, MNRAS, 497, 1015

Fuhrmann, K., Pfeiffer, M. J., \& Bernkopf, J. 1998, A\&A, 336, 942

Fujii, Y., Spiegel, D. S., Mroczkowski, T., et al. 2016, ApJ, 820, 122

George, S. J., \& Stevens, I. R. 2007, MNRAS, 382, 455

Green, D. A., \& Madhusudhan, N. 2021, MNRAS, 500, 211

Grießmeier, J.-M. 2015, Astrophys. Space Sci. Lib., 411, 213

Grießmeier, J.-M. 2017, Planetary Radio Emissions VIII, eds. G. Fischer G. Mann, M. Panchenko, \& P. Zarka (Vienna: Austrian Academy of Sciences Press), 285

Griessmeier, J. M. 2018, Handbook of Exoplanets (Berlin: Springer), 159

Grießmeier, J.-M., Stadelmann, A., Motschmann, U., et al. 2005a, Astrobiology, 5,587

Grießmeier, J.-M., Motschmann, U., Mann, G., \& Rucker, H. O. 2005b, A\&A, 437, 717

Grießmeier, J.-M., Zarka, P., \& Spreeuw, H. 2007a, A\&A, 475, 359

Grießmeier, J. M., Preusse, S., Khodachenko, M., et al. 2007b, Planet. Space Sci., 55,618

Grießmeier, J.-M., Stadelmann, A., Grenfell, J. L., Lammer, H., \& Motschmann, U. 2009, Icarus, 199, 526

Grießmeier, J.-M., Tabataba-Vakili, F., Stadelmann, A., Grenfell, J. L., \& Atri, D. 2015, A\&A, 581, A44

Grießmeier, J.-M., Tabataba-Vakili, F., Stadelmann, A., Grenfell, J. L., \& Atri, D. 2016, A\&A, 587, A159

Güdel, M. 2002, ARA\&A, 40, 217

Gurumath, S. R., Hiremath, K. M., \& Ramasubramanian, V. 2018, IAU Symp. 340,242

Hallinan, G., Sirothia, S. K., Antonova, A., et al. 2013, ApJ, 762, 34

Heald, G. H., Pizzo, R. F., Orrú, E., et al. 2015, A\&A, 582, A123

Helfand, D. J., White, R. L., \& Becker, R. H. 2015, ApJ, 801, 26

Hess, S. L. G., \& Zarka, P. 2011, A\&A, 531, A29

Howard, W. S., Corbett, H., Law, N. M., et al. 2019, ApJ, 881, 9

Hubbard, W. B., \& Smoluchowski, R. 1973, Space Sci. Rev., 14, 599

Intema, H. T., van der Tol, S., Cotton, W. D., et al. 2009, A\&A, 501, 1185

Intema, H. T., Jagannathan, P., Mooley, K. P., \& Frail, D. A. 2017, A\&A, 598, A78

Jardine, M., \& Collier Cameron, A. 2008, A\&A, 490, 843

Jeffers, S. V., Mengel, M., Moutou, C., et al. 2018, MNRAS, 479, 5266

Justesen, A. B., \& Albrecht, S. 2019, A\&A, 625, A59

Kao, M. M., Hallinan, G., Pineda, J. S., et al. 2016, ApJ, 818, 24

Kao, M. M., Hallinan, G., Pineda, J. S., Stevenson, D., \& Burgasser, A. 2018 , ApJS, 237, 25

Karako-Argaman, C., Kaspi, V. M., Lynch, R. S., et al. 2015, ApJ, 809, 67

Kasting, J. 2010, How to Find a Habitable Planet (Princeton: Princeton University Press)

Kavanagh, R. D., Vidotto, A. A., Ó. Fionnagáin, D., et al. 2019, MNRAS, 485, 4529

Kislyakova, K. G., Pilat-Lohinger, E., Funk, B., et al. 2016, MNRAS, 461, 988

Kopp, A., Schilp, S., \& Preusse, S. 2011, ApJ, 729, 116

Lai, D., Helling, C., \& van den Heuvel, E. P. J. 2010, ApJ, 721, 923

Lammer, H., Bredehöft, J. H., Coustenis, A., et al. 2009, A\&AR, 17, 181

Lamy, L., Zarka, P., Cecconi, B., et al. 2017, in Planetary Radio Emissions VIII, eds. G. Fischer, G. Mann, M. Panchenko, \& P. Zarka, 455

Lamy, L., Zarka, P., Cecconi, B., et al. 2018, Science, 362, aat2027

Landsman, W. B. 1995, ASP Conf. Ser., 77, 437

Laughlin, G. 2018, Handbook of Exoplanets (Cham: Springer), 1

Law, N. M., Fors, O., Ratzloff, J., et al. 2015, PASP, 127, 234

Lazio, T. J. W. 2018, Handbook of Exoplanets (Cham: Springer), 9

Lazio, T. J. W., \& Farrell, W. M. 2007, ApJ, 668, 1182

Lazio, W., T. J., Farrell, W. M., Dietrick, J., et al. 2004, ApJ, 612, 511

Lazio, T. J. W., Carmichael, S., Clark, J., et al. 2010a, AJ, 139, 96

Lazio, T. J. W., Shankland, P. D., Farrell, W. M., \& Blank, D. L. 2010b, AJ, 140, 1929

Lazio, T. J. W., Shkolnik, E., Hallinan, G., \& Planetary Habitability Study Team. 2016, Planetary Magnetic Fields: Planetary Interiors and Habitability, Tech. rep.

Lazio, J., Hallinan, G., Airapetian, A., et al. 2019, BAAS, 51, 135

Lecavelier Des Etangs, A., Sirothia, S. K., Gopal-Krishna, \& Zarka, P. 2009, A\&A, 500, L51

Lecavelier Des Etangs, A., Sirothia, S. K., Gopal-Krishna, \& Zarka, P. 2011, A\&A, 533, A50

Lecavelier des Etangs, A., Sirothia, S. K., Gopal-Krishna, \& Zarka, P. 2013, A\&A, 552, A65

Lenc, E., Murphy, T., Lynch, C. R., Kaplan, D. L., \& Zhang, S. N. 2018, MNRAS, 478,2835

Llama, J., Wood, K., Jardine, M., et al. 2011, MNRAS, 416, L41
Loh, A., Lamy, L., Zarka, P., et al. 2017, SF2A-2017: Annual meeting of the French Society of Astronomy and Astrophysics http://sf2a.eu/ semaine-sf2a/2017/posterpdfs/267_200_54.pdf

Louis, C. K., Hess, S. L. G., Cecconi, B., et al. 2019, A\&A, 627, A30

Lynch, C. R., Murphy, T., Kaplan, D. L., Ireland, M., \& Bell, M. E. 2017, MNRAS, 467, 3447

Lynch, C. R., Murphy, T., Lenc, E., \& Kaplan, D. L. 2018, MNRAS, 478, 1763

Manchester, R. N., Hobbs, G. B., Teoh, A., \& Hobbs, M. 2005, AJ, 129, 1993

Marques, M. S., Zarka, P., Echer, E., et al. 2017, A\&A, 604, A17

McIntyre, S. R. N., Lineweaver, C. H., \& Ireland , M. J. 2019, MNRAS, 485, 3999

Mengel, M. W., Fares, R., Marsden, S. C., et al. 2016, MNRAS, 459, 4325

Mertens, F. G., Mevius, M., Koopmans, L. V. E., et al. 2020, MNRAS, 493, 1662

Miller, B. P., Gallo, E., Wright, J. T., \& Dupree, A. K. 2012, ApJ, 754, 137

Miller, B. P., Gallo, E., Wright, J. T., \& Pearson, E. G. 2015, ApJ, 799, 163

Million, M. A., \& Goertz, C. K. 1988, Geophys. Res. Lett., 15, 111

Mittag, M., Robrade, J., Schmitt, J. H. M. M., et al. 2017, A\&A, 600, A119

Mottez, F., \& Zarka, P. 2014, A\&A, 569, A86

Murphy, T., Bell, M. E., Kaplan, D. L., et al. 2015, MNRAS, 446, 2560

Narang, M., Manoj, P., Chandra, C. H. I., et al. 2021, MNRAS, 500, 4818

Nichols, J. D. 2011, MNRAS, 414, 2125

Nichols, J. D. 2012, MNRAS, 427, L75

Nichols, J. D., \& Milan, S. E. 2016, MNRAS, 461, 2353

Nicholson, B. A., Vidotto, A. A., Mengel, M., et al. 2016, MNRAS, 459, 1907

Offringa, A. R. 2012, PhD thesis, University of Groningen https:// www.rug.nl/research/portal/publications/pub(b427903c-bf234bd5-b641-a58993f54291).html

Offringa, A. R., de Bruyn, A. G., Biehl, M., et al. 2010, MNRAS, 405, 155

Offringa, A. R., van de Gronde, J. J., \& Roerdink, J. B. T. M. 2012, A\&A, 539, A95

O'Gorman, E., Coughlan, C. P., Vlemmings, W., et al. 2018, A\&A, 612, A52

Oklopčić, A., Silva, M., Montero-Camacho, P., \& Hirata, C. M. 2020, ApJ, 890, 88

Owen, J. E., \& Adams, F. C. 2014, MNRAS, 444, 3761

Perna, R., Menou, K., \& Rauscher, E. 2010, ApJ, 719, 1421

Pick, M., \& Vilmer, N. 2008, A\&ARv, 16, 1

Pilia, M., Hessels, J. W. T., Stappers, B. W., et al. 2016, A\&A, 586, A92

Piskorz, D., Benneke, B., Crockett, N. R., et al. 2017, AJ, 154, 78

Pont, F., Zucker, S., \& Queloz, D. 2006, MNRAS, 373, 231

Pope, B. J. S., Bedell, M., Callingham, J. R., et al. 2020, ApJ, 890, L19

Poppenhaeger, K., \& Wolk, S. J. 2014, A\&A, 565, L1

Preusse, S., Kopp, A., Büchner, J., \& Motschmann, U. 2006, A\&A, 460, 317

Ratzloff, J. K., Law, N. M., Fors, O., et al. 2019, PASP, 131, 075001

Rauscher, E., \& Menou, K. 2013, APJ, 764, 103

Reiners, A., \& Christensen, U. R. 2010, A\&A, 522, A13

Rodler, F., Lopez-Morales, M., \& Ribas, I. 2012, ApJ, 753, L25

Rogers, T. M., \& Komacek, T. D. 2014, ApJ, 794, 132

Route, M. 2019, ApJ, 872, 79

Ryabov, V. B., Zarka, P., \& Ryabov, B. P. 2004, Planet. Space Sci., 52, 1479

Sánchez-Lavega, A. 2004, ApJ, 609, L87

Saumon, D., \& Marley, M. S. 2008, ApJ, 689, 1327

Saur, J., Grambusch, T., Duling, S., Neubauer, F. M., \& Simon, S. 2013, A\&A, 552, A119

Schneider, J., Dedieu, C., Le Sidaner, P., Savalle, R., \& Zolotukhin, I. 2011, A\&A, 532, A79

See, V., Jardine, M., Fares, R., Donati, J.-F., \& Moutou, C. 2015, MNRAS, 450, 4323

Shimwell, T. W., Röttgering, H. J. A., Best, P. N., et al. 2017, A\&A, 598, A104

Shimwell, T. W., Tasse, C., Hardcastle, M. J., et al. 2019, A\&A, 622, A1

Shiohira, Y., Terada, Y., Mukuno, D., Fujii, Y., \& Takahashi, K. 2020, MNRAS, 495, 1934

Shiratori, Y., Yokoo, H., Sasao, T., et al. 2006, Tenth Anniversary of 51 Peg-b : Status of and Prospects for hot Jupiter studies, eds. L. Arnold, F. Bouchy, \& C. Moutou (Platypus Press), 290

Shkolnik, E., Walker, G. A. H., \& Bohlender, D. A. 2003, ApJ, 597, 1092

Shkolnik, E., Walker, G. A. H., Bohlender, D. A., Gu, P.-G., \& Kürster, M. 2005, ApJ, 622, 1075

Shkolnik, E., Bohlender, D. A., Walker, G. A. H., \& Collier Cameron A. 2008, ApJ, 676, 628

Sirothia, S. K., Lecavelier des Etangs, A., Gopal-Krishna, Kantharia, N. G., \& Ishwar-Chandra, C. H. 2014, A\&A, 562, A108

Smith, A. M. S., Collier Cameron, A., Greaves, J., et al. 2009, MNRAS, 395, 335

Stappers, B. W., Hessels, J. W. T., Alexov, A., et al. 2011, A\&A, 530, A80

Stevens, I. R. 2005, MNRAS, 356, 1053

Stevenson, D. J. 2003, Earth Planet. Sci. Lett., 208, 1

Stroe, A., Snellen, I. A. G., \& Röttgering, H. J. A. 2012, A\&A, 546, A116

Treumann, R. A. 2006, A\&ARv, 13, 229 
Turner, J. D., Christie, D., Arras, P., Johnson, R. E., \& Schmidt, C. 2016a, MNRAS, 458, 3880

Turner, J. D., Pearson, K. A., Biddle, L. I., et al. 2016b, MNRAS, 459, 789

Turner, J. D., Grießmeier, J.-M., Zarka, P., \& Vasylieva, I. 2017a, Planetary Radio Emissions VIII, eds. G. Fischer, G. Mann, M. Panchenko, \& P. Zarka (Vienna: Austrian Academy of Sciences Press), 301

Turner, J. D., Leiter, R. M., Biddle, L. I., et al. 2017b, MNRAS, 472, 3871

Turner, J. D., Grießmeier, J.-M., Zarka, P., \& Vasylieva, I. 2019, A\&A, 624, A40 van Haarlem, M. P., Wise, M. W., Gunst, A. W., et al. 2013, A\&A, 556, A2

Vasylieva, I. 2015, Theses, Paris Observatory. https://tel.archivesouvertes.fr/tel-01246634

Vedantham, H. K., Callingham, J. R., Shimwell, T. W., et al. 2020, Nat. Astron. 4, 577

Vidotto, A. A., Fares, R., Jardine, M., et al. 2012, MNRAS, 423, 3285

Vidotto, A. A., Fares, R., Jardine, M., Moutou, C., \& Donati, J.-F. 2015, MNRAS 449,4117

Vidotto, A. A., Jardine, M., \& Helling, C. 2010a, ApJ, 722, L168

Vidotto, A. A., Opher, M., Jatenco-Pereira, V., \& Gombosi, T. I. 2010b, ApJ, 720, 1262

Vidotto, A. A., Jardine, M., \& Helling, C. 2011, MNRAS, 411, L46

Walker, G. A. H., Croll, B., Matthews, J. M., et al. 2008, A\&A, 482, 691

Wang, J., \& Ford, E. B. 2011, MNRAS, 418, 1822

Wang, X., \& Loeb, A. 2019, ApJ, 874, L23

Warwick, J. W., Evans, D. R., Romig, J. H., et al. 1986, Science, 233, 102

Warwick, J. W., Evans, D. R., Peltzer, G. R., et al. 1989, Science, 246, 1498

Weber, C., Erkaev, N. V., Ivanov, V. A., et al. 2018, MNRAS, 480, 3680

Weber, C., Lammer, H., Shaikhislamov, I. F., et al. 2017a, MNRAS, 469, 3505

Weber, C., Lammer, H., Shaikhislamov, I. F., et al. 2017b, Planetary Radio Emis sions VIII, eds. G. Fischer, G. Mann, M. Panchenko, \& P. Zarka (Vienna: Austrian Academy of Sciences Press), 317
Winglee, R. M., Dulk, G. A., \& Bastian, T. S. 1986, ApJ, 309, L59

Wu, C. S., \& Lee, L. C. 1979, ApJ, 230, 621

Yantis, W. F., Sullivan, III, W. T., \& Erickson, W. C. 1977, BAAS, 9, 453

Zakharenko, V. V., Vasylieva, I. Y., Konovalenko, A. A., et al. 2013, MNRAS, 431,3624

Zarka, P. 1992, Adv. Space Res., 12, 99

Zarka, P. 1998, J. Geophys. Res., 103, 20159

Zarka, P. 2004, Planet. Space Sci., 52, 1455

Zarka, P. 2007, Planet. Space Sci., 55, 598

Zarka, P. 2010, ASP Conf. Ser., 430, 175

Zarka, P. 2011, Planetary Radio Emissions VII, eds. H. O. Rucker, W. S. Kurth, P. Louarn, \& G. Fischer (Vienna: Austrian Academy of Sciences Press), 287 Zarka, P. 2018, Handbook of Exoplanets, (Cham: Springer) 22

Zarka, P., Farges, T., Ryabov, B. P., Abada-Simon, M., \& Denis, L. 1996, Geophys. Res. Lett., 23, 125

Zarka, P., Queinnec, J., Ryabov, B. P., et al. 1997, Planetary Radio Emission IV, eds. H. O. Rucker, S. J. Bauer, \& A. Lecacheux, 101

Zarka, P., Treumann, R. A., Ryabov, B. P., \& Ryabov, V. B. 2001, Astrophys. Space. Sci., 277, 293

Zarka, P., Cecconi, B., \& Kurth, W. S. 2004, J. Geophys. Res. Space Phys., 109, A09S15

Zarka, P., Girard, J. N., Tagger, M., \& Denis, L. 2012, SF2A-2012: Proceedings of the Annual meeting of the French Society of Astronomy and Astrophysics, eds. S. Boissier, P. de Laverny, N. Nardetto, R. Samadi, D. Valls-Gabaud, \& H. Wozniak, 687

Zarka, P., Lazio, J., \& Hallinan, G. 2015, Advancing Astrophysics with the Square Kilometre Array (AASKA14), 120

Zarka, P., Marques, M. S., Louis, C., et al. 2018, A\&A, 618, A84

Zarka, P., Denis, L., Tagger, M., et al. 2020, in URSI General Assembly, session J01: New Telescopes on the Frontier 


\section{Appendix A: Observational setup}

Table A.1 gives the exact dates, times and observation IDs for each one of our observations. It also includes the number of stations that were found to behave non-optimally during the observation, and the an assessment of the data quality (percentage of data masked during RFI flagging).
Table A.1 shows that we observed the pulsar B0809+74 for 10 or 15 min either before or after each of the $v$ And and $\tau$ Boo observations. For these observations, we used the same settings as the exoplanet observation. These pulsar observations were taken to test and calibrate the processing pipeline. Some of these tests are shown in Appendix G.

Table A.1. Summary of the observations.

\begin{tabular}{|c|c|c|c|c|c|c|c|}
\hline Obs \# & LOFAR cycle & LOFAR ID & $\begin{array}{l}\text { Date and time } \\
\text { (UTC) }\end{array}$ & $\begin{array}{c}\text { Duration } \\
(\mathrm{h})\end{array}$ & $\begin{array}{c}\text { Elevation } \\
\left({ }^{\circ}\right) \\
\end{array}$ & $\begin{array}{c}\text { Bad stations } \\
(\#)\end{array}$ & $\begin{array}{c}\text { RFI masked } \\
(\%)\end{array}$ \\
\hline \multicolumn{8}{|c|}{$55 \mathrm{Cnc}[24 \mathrm{~h}]$} \\
\hline 1 & 5 & L429868 & 2016-02-03 20:00 & 8 & $37-65$ & 2 & 4.5 \\
\hline- & 5 & L432752 (1) & 2016-02-27 18:03 & 9 & $30-65$ & 2 & 100 \\
\hline 2 & 5 & L433872 & 2016-03-02 19:00 & 4 & $52-65$ & 2 & 3.4 \\
\hline 3 & 5 & L441630 & 2016-03-28 18:00 & 6 & $40-65$ & 2 & 5.9 \\
\hline 4 & 5 & L527649 & 2016-07-31 08:00 & 3 & $40-60$ & 2 & 7.1 \\
\hline 5 & 5 & L554093 & 2016-10-22 03:00 & 3 & $45-65$ & 1 & 5.7 \\
\hline \multicolumn{8}{|c|}{$v$ And $[45 \mathrm{~h}]$} \\
\hline 1 & 6 & L545197 & 2016-09-08 00:00 & 5 & $58-78$ & 2 & 4.7 \\
\hline 2 & 6 & L545213 & 2016-09-09 00:00 & 5 & $58-78$ & 1 & 2.9 \\
\hline 3 & 6 & L545209 & 2016-09-10 00:00 & 5 & $58-78$ & 1 & 5.2 \\
\hline 4 & 6 & L547657 & 2016-09-24 22:00 & 5 & $58-78$ & 1 & 3.4 \\
\hline 5 & 6 & L547653 & 2016-09-25 23:00 & 5 & $55-78$ & 1 & 3.4 \\
\hline 6 & 6 & L547649 & 2016-09-26 22:00 & 5 & $58-78$ & 1 & 4.1 \\
\hline 7 & 6 & L547645 & 2016-09-28 23:00 & 5 & $55-78$ & 1 & 4.0 \\
\hline 8 & 6 & L551195 & 2016-10-10 22:02 & 5 & $55-78$ & 2 & 4.3 \\
\hline 9 & 6 & L552145 & 2016-10-13 22:00 & 5 & $55-78$ & 2 & 5.3 \\
\hline \multicolumn{8}{|c|}{$\tau$ Boo $[20 \mathrm{~h}]$} \\
\hline 1 & 7 & L569131 & 2017-02-18 01:12 & 3 & $45-53$ & 2 & 6.3 \\
\hline 2 & 7 & L569127 & 2017-02-22 01:00 & 2.5 & $45-55$ & 2 & 4.6 \\
\hline 3 & 7 & L569123 & 2017-02-26 01:16 & 3 & $48-55$ & 2 & 5.6 \\
\hline 4 & 7 & L569119 & 2017-02-27 01:16 & 2.5 & $48-55$ & 2 & 6.2 \\
\hline 5 & 7 & L570729 & 2017-03-01 01:16 & 3 & $50-55$ & 2 & 6.4 \\
\hline 6 & 7 & L570725 & 2017-03-06 01:00 & 3 & $50-55$ & 1 & 4.7 \\
\hline 7 & 7 & L581807 & 2017-03-25 01:00 & 3 & $40-55$ & 2 & 4.8 \\
\hline \multicolumn{8}{|c|}{ B0809+74 [197 min] } \\
\hline 1 & 6 & L545199 & 2016-09-07 23:49 & 0.17 & 38 & 2 & 2.5 \\
\hline 2 & 6 & L545215 & 2016-09-08 23:49 & 0.17 & 38 & 1 & 3.1 \\
\hline 3 & 6 & L545211 & 2016-09-09 23:49 & 0.17 & 38 & 1 & 4.5 \\
\hline 4 & 6 & L547659 & 2016-09-24 21:49 & 0.17 & 38 & 1 & 3.2 \\
\hline 5 & 6 & L547655 & 2016-09-25 22:49 & 0.17 & 39 & 1 & 2.8 \\
\hline 6 & 6 & L547651 & 2016-09-26 21:49 & 0.17 & 38 & 1 & 6.1 \\
\hline 7 & 6 & L547647 & 2016-09-28 22:49 & 0.17 & 39 & 1 & 2.8 \\
\hline 8 & 6 & L551197 & 2016-10-11 03:03 & 0.17 & 52 & 2 & 6.2 \\
\hline 9 & 6 & L552143 & 2016-10-13 21:49 & 0.17 & 39 & 2 & 5.9 \\
\hline 10 & 7 & L569129 & 2017-02-18 00:56 & 0.25 & 67 & 2 & 5.9 \\
\hline 11 & 7 & L569125 & 2017-02-22 00:44 & 0.25 & 67 & 2 & 2.9 \\
\hline 12 & 7 & L569121 & 2017-02-26 01:00 & 0.25 & 65 & 2 & 4.4 \\
\hline 13 & 7 & L569117 & 2017-02-27 01:00 & 0.25 & 65 & 2 & 4.9 \\
\hline 14 & 7 & L570727 & 2017-03-01 01:00 & 0.25 & 65 & 2 & 3.9 \\
\hline 15 & 7 & L570723 & 2017-03-06 00:44 & 0.25 & 64 & 1 & 3.5 \\
\hline 16 & 7 & L581805 & 2017-03-25 00:44 & 0.25 & 60 & 2 & 4.1 \\
\hline
\end{tabular}

Notes. Column 1: observation number. Column 2: LOFAR cycle. Column 3: LOFAR observation ID. Column 4: date and start time of the observation (UTC). Column 5: duration of the observation. Column 6: target elevation range. Column 7: number of bad stations present during the observation found by examining the station inspection plots. Column 8: amount of RFI masked by the BOREALIS pipeline. (1) This observation was not use in the analysis due to near-continuous RFI at all frequencies for the entire observing period. In addition, during this observation, there was also an antenna cable delay compensation issue (communication from ASTRON Radio Observatory staff). 
Table A.2. Beam coordinates used for the observations.

\begin{tabular}{cccc}
\hline \hline Beam & $\begin{array}{c}\text { RA (J2000) } \\
(\mathrm{h}: \mathrm{m}: \mathrm{s})\end{array}$ & $\begin{array}{c}\text { Dec }(\mathrm{J} 2000) \\
\left({ }^{\circ}:^{\prime}: "\right)\end{array}$ & $\begin{array}{c}\text { Distance } \\
\left({ }^{\circ}\right)\end{array}$ \\
\hline \multicolumn{4}{c}{$55 \mathrm{Cnc}$} \\
\hline ON & $08: 52: 34.81$ & $+28: 19: 51.00$ & - \\
OFF 1 & $08: 26: 51.38$ & $+26: 37: 23.80$ & 6.0 \\
OFF 2 & $08: 58: 10.14$ & $+27: 50: 53.09$ & 1.3 \\
\hline \multicolumn{4}{c}{$v$ And } \\
\hline ON & $01: 36: 47.84$ & $+41: 24: 19.60$ & - \\
OFF 1 & $01: 40: 00$ & $+38: 00: 00$ & 3.4 \\
OFF 2 & $01: 30: 00$ & $+48: 00: 00$ & 6.7 \\
\hline \multicolumn{4}{c}{$\tau$ Boo } \\
\hline ON & $13: 47: 15.74$ & $+17: 27: 24.90$ & - \\
OFF 1 & $13: 54: 44.953$ & $+16: 49: 29.20$ & 1.9 \\
OFF 2 & $13: 58: 10.366$ & $+19: 00: 01.37$ & 3.0 \\
\hline \multicolumn{4}{c}{ B0809+74 } \\
\hline ON & $08: 14: 59.52$ & $+74: 29: 06.00$ \\
OFF 1 & $08: 23: 24.48$ & $+71: 52: 58.80$ & - \\
OFF 2 & $09: 03: 31.92$ & $+74: 52: 58.80$ & 3.2 \\
\hline
\end{tabular}

Notes. Column 1: beam name. Column 2: right ascension (RA) of the beam. Column 3: declination (Dec) of the beam. Column 4: distance of the beam from the ON-beam.

For all observations, the beam coordinates of the $\mathrm{ON}$ and OFF beams are given in Table A.2. For the observations of $55 \mathrm{Cnc}$, the positions of the two OFF beams correspond to the position of the pulsar B0823+26 (OFF beam 1) and at a nearby "empty" sky region (OFF beam 2). For the $v$ And and $\tau$ Boo observations, the two OFF beams correspond to "empty" sky patches, i.e. positions without point sources at a level $\geq 100 \mathrm{mJy}$ according to the LOFAR MSSS-HBA survey $(150 \mathrm{MHz}$, Heald et al. 2015) and without point sources at $\geq 5 \mathrm{mJy}$ in the TGSS survey (150 MHz, Intema et al. 2017).

\section{Appendix B: Observable quantities}

The post-processing section of the BOREALIS pipeline computes a series of observable quantities, Q1 to Q4. As defined in T19, Q1 is designed to search for slowly variable emission on the order of minutes to hours. Q2 is the frequency-integrated time-series designed to find bursty emission and is created by integrating the processed data over a selected frequency range (several trial values were used, see Table 3), followed by highpass filtering using a smoothing window of 10 time bins (e.g $10 \mathrm{~s}$ for a rebin time of $1 \mathrm{~s}$ ) and normalization by the standard deviation. Q3 is designed to localize the bursts (Q2) in time with a resolution $\delta T$ (e.g., $2 \mathrm{~min}$ ) for one selected threshold $\eta$ (in units of standard deviations). Q4 is the statistical analysis of the broadband burst emission (Q2), searched over an entire observation and a selected frequency range, and plotted against the threshold $\eta$.

For convenience, the definition of those each observable quantities that are used in the present work is summarised below: - Q1: slowly variable emission observables.

- Q1a (Time-series): dynamic spectrum integrated over all frequencies and rebinned to a time resolution of $\delta T$ (e.g., 2 min)
- Q1b (Integrated spectrum): dynamic spectrum integrated over all times and rebinned to a frequency resolution of $\delta F$ (e.g., $0.5 \mathrm{MHz}$ )

- Q2 : time series obtained by integrating the processed data over a selected frequency range (several trial values were used, see Table 3), followed by high-pass filtering created using a smoothing window of 10 time bins (e.g., $10 \mathrm{~s}$ for a rebin time of $1 \mathrm{~s}$ ) and normalization by the standard deviation.

- Q4: statistical analysis of the broadband burst emission (Q2), searched over an entire observation and a selected frequency range, and plotted against the threshold $\eta$ (in units of standard deviations).

- Q4a (Number of Peaks): number of peaks where Q2 $\geq \eta$.

- Q4b (Power of Peaks): sum of the power of peaks where $\mathrm{Q} 2 \geq \eta$.

- Q4c (Peak Asymmetry): number of peaks where Q2 $\geq \eta$ subtracted by the number of peaks where $\mathrm{Q} 2 \leq-\eta$.

- Q4d (Power Asymmetry): sum of the power of peaks where $\mathrm{Q} 2 \geq \eta$ subtracted by the sum of the |power| of peaks where $\mathrm{Q} 2 \leq-\eta$.

- Q4e (Peak Offset): number of peaks where Q2 $\geq \eta$ for the ON-beam and exceeding the corresponding OFF values by a factor $\geq 2$.

- Q4f (Power Offset): sum of the power of peaks where Q2 $\geq \eta$ for the ON-beam and exceeding the corresponding OFF values by a factor $\geq 2$.

When examining Q4a-f, we compare two beam against each other in the form of difference curves, e.g. Q4f $\mathrm{f}_{\text {Diff }}=\mathrm{Q} 4 \mathrm{f}(\mathrm{ON})$ $\mathrm{Q} 4 \mathrm{f}(\mathrm{OFF})$ or $\mathrm{Q} 4 \mathrm{f}_{\text {Diff }}=\mathrm{Q} 4 \mathrm{f}(\mathrm{OFF} 1)-\mathrm{Q} 4 \mathrm{f}(\mathrm{OFF} 2)$.

The post-processing pipeline produces Q1-Q4 synthesis plots, for each observation of Table A.1, pair of beams, and set of parameters of Table 3. Example Q1, Q2, and Q4 plots can be found in Figs. 4 and 5. Figure 5 shows the Q1 observables (Q1a in Fig. 5a and Q1b in Fig. 5b). The ON beam is displayed in black, and the OFF-beams are displayed in red and blue. The lower panel show the differences between two beams (orange, green, and brown curves). Figures $4 \mathrm{a}$ and $\mathrm{b}$ display the observable quantity Q2 in the form of scatter plots. These scatter plots allow to compare two beams by showing the normalized signal in one beam versus the normalized signal in the other. Figures 4c-f display the Q4a, Q4b, Q4e, and Q4f observables for the ON-OFF and OFF1-OFF2 difference curves. In these plots, the 3 pairs of dashed curves surrounding the horizontal axis indicate the $\pm 1, \pm 2$ and $\pm 3 \sigma$ levels reached when computing these Q4 observables from 10000 pairs of Gaussian noise time series, as a function of the threshold on normalized signal intensity. Q4 difference curves exceeding the $2 \sigma$ to $3 \sigma$ reference curves over a significant range of thresholds indicate a possible detection. We consider the observables Q4a-f separately.

In most observations, the distribution of points in the Q2 scatter plot is not as "circular" as it should be if both signals were Gaussian. Ionospheric fluctuations affecting both the ONand the OFF-beam simultaneously may elongate the scatter-plot along the main diagonal and thus cause cause a non-circular distribution. To remedy this problem, an elliptical correction was designed (T19). It circularizes the distribution of points in the Q2 scatter plot (i.e. mitigates the ionospheric fluctuations), thus allowing to detect more easily outliers which appear only in the ON-beam. 


\section{Appendix C: Detection criteria}

At the end of Sect. 4, we showed the criteria that are used to determine a tentative detection. These criteria are based on the observable quantities (Q1a, Q1b, Q2, and Q4a-f, as defined in Appendix B). In this appendix, we describe how these criteria are implemented in an automated search algorithm.

\section{C.1. Criteria for slowly varying emission (observable quantities Q1a and Q1b)}

For the observables Q1a and Q1b, we define the following detection criteria (sometimes also called "summary numbers"):

- N1: integral of the ON-OFF difference curve.

$-N 2$ : percentage of positive values in the ON-OFF difference curve.

- N3: standard deviation of the ON-OFF difference curve.

- N4: probability of false-detection, comprised between 0 and 1. It describes the likelihood that the ON-OFF difference curve can be reproduced from simulations based on random Gaussian noise. For example, a probability of $6.3 \times 10^{-5}$ corresponds to a $4 \sigma$ detection.

The detection criteria $N 1$ to $N 3$ should increase if there is an excess signal in the ON-beam.

\section{C.2. Criteria for burst emission (observable quantities Q4a to Q4f)}

For the observables Q4a to Q4f, we define the following detection criteria:

- N5: integral of the ON-OFF difference curve.

- N6: ratio of $N 5$ to the integral of the $1 \sigma$ reference Gaussian curve (e.g., dashed lines in Fig. C.2). The reference curve was created by running simulations on 10000 pairs of Gaussian noise distributions and calculating the respective observable (i.e. Q4f).

- N7: percentage of positive values in the ON-OFF difference curve.

- N8: percentage of ON-OFF values above the $1 \sigma$ reference Gaussian curve.

- N9: minimum $\left(\eta_{\min }\right)$ and maximum $\left(\eta_{\max }\right)$ threshold values where $\mathrm{ON}-\mathrm{OFF}>0$

- N10: percentage of positive values in the ON-OFF difference curve for $\eta_{\min } \leq \eta \leq \eta_{\max }$

- N11: percentage of negative values in the ON-OFF difference curve for $\eta \geq \eta_{\max }$

- N12: probability of false-detection, comprised between 0 and 1 (similar to N4).

If an excess signal is in the ON-beam then the detection criteria $N 5$ and $N 6$ should both be greater than 0 . The detection criteria $N 5, N 6$, and $N 10$ probe the continue nature of the emission which is essential for a detection. Additionally, $N 11$ can be used to eliminate false-positives because $N 11=0$ for a detection.

\section{C.3. Test of the detection criteria}

In order to determine the reliability of the detection criteria in finding a signal, we tested them on LOFAR data containing radio emission from Jupiter. We used the LOFAR dataset of Jupiter radio emission attenuated by a factor $\alpha=10^{-3}$ from T19. An example Q1a difference curve can be seen in Fig. C.1 and the corresponding detection criteria from these plots are listed in Table C.1. All the Q1 detection criteria show an excess in the a.)

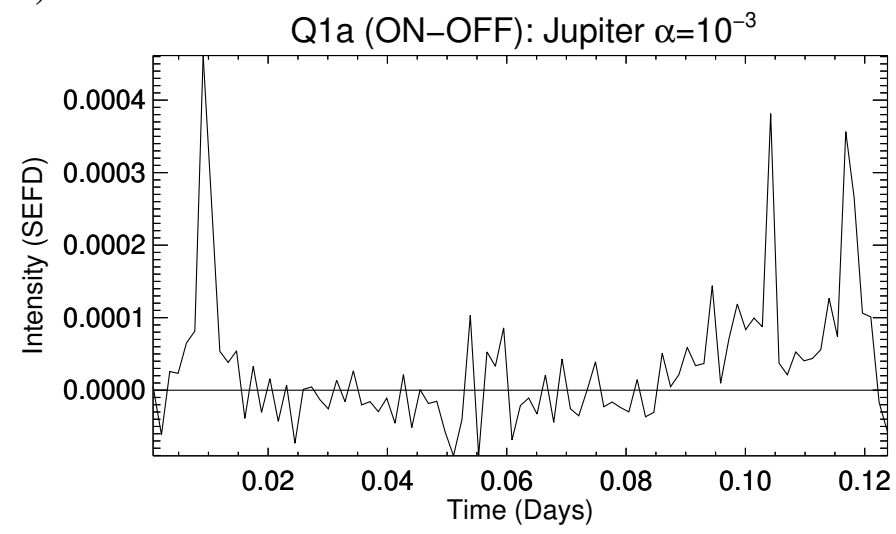

b.)

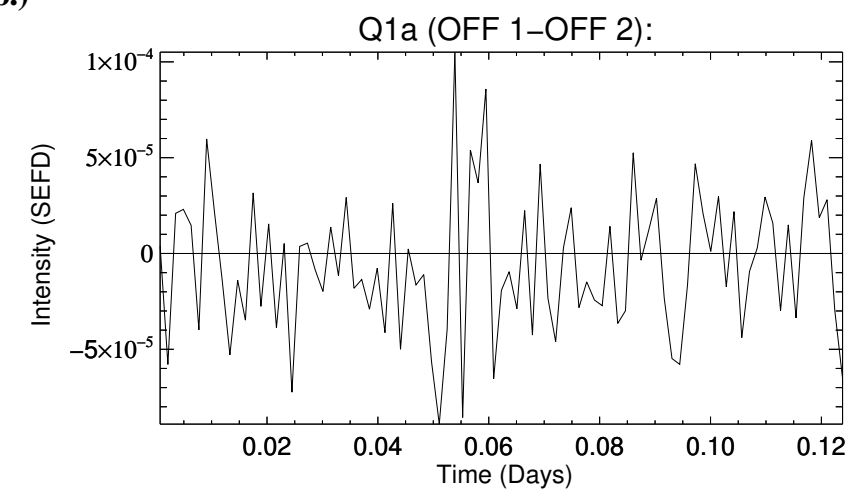

Fig. C.1. Example Q1a difference curves for Jupiter signal attenuated by a factor $\alpha=10^{-3}$ (panel $a$ ) and for the two OFF beams (panel $b$ ).

Table C.1. Values of detection criteria for Q1a from Fig. C.1.

\begin{tabular}{cccc}
\hline \hline$\#$ & ON- OFF & OFF 1- OFF 2 & |Ratio| \\
\hline N1 & $3.3 \times 10^{-6}$ & $-1.2 \times 10^{-6}$ & 2.75 \\
N2 & 60 & 44 & 1.35 \\
N3 & $9.4 \times 10^{-5}$ & $3.7 \times 10^{-5}$ & 2.54 \\
N4 & $10^{-4},>3.8 \sigma$ & $1,1 \sigma$ & $3.8 \sigma$ \\
\hline
\end{tabular}

Notes. Column 1: detection criterion. Column 2: detection criterion applied to the difference between ON and OFF beam. Column 3: detection criterion applied to the difference between OFF1 and OFF2 beam. Column 4: absolute value of the ratio of ON-OFF (Col. 2) by OFF1-OFF2 (Col. 3).

ON-beam. The excess is moderate because the emission essentially consisted of two burst, one near the beginning and one ear the end of the interval (see Fig. 1 in Turner et al. 2019). From $N 4$, we conclude this is a real detection at $3.8 \sigma$ significance.

An example Q4f difference plot is shown in Fig. C.2; the corresponding detection criteria are listed in Table C.2. We note that the line in the plot for $\eta_{\max }$ and $\eta_{\min }$ are slightly offset due to the granularity of the simulations in $\eta$-space. In Table C.2, all detection criteria show an excess in the ON-beam with very large ratios for $N 5$ and $N 6 . N 6$ also probes the continuous nature of the curve which is essential for detection. N12 is also very useful because it allows us to check the false positive rate of any tentative detections. From these detection criteria. we conclude a firm detection of bursty emission in this dataset. 
a.)

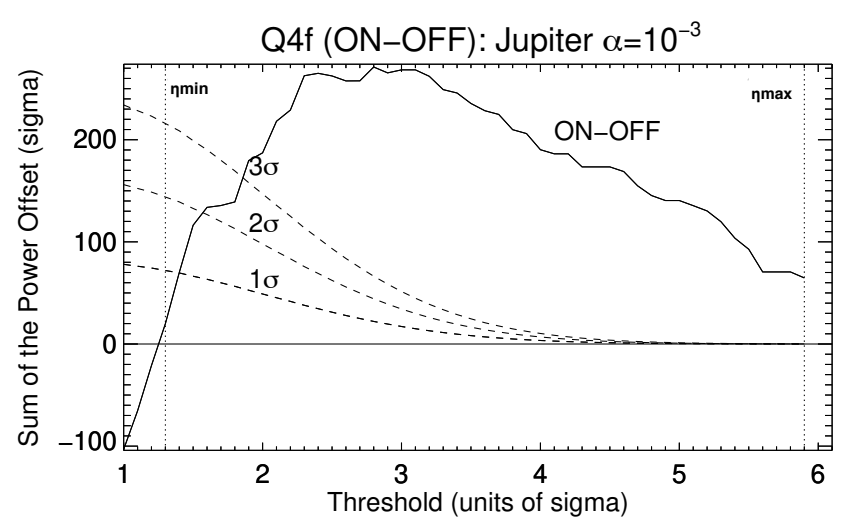

b.)

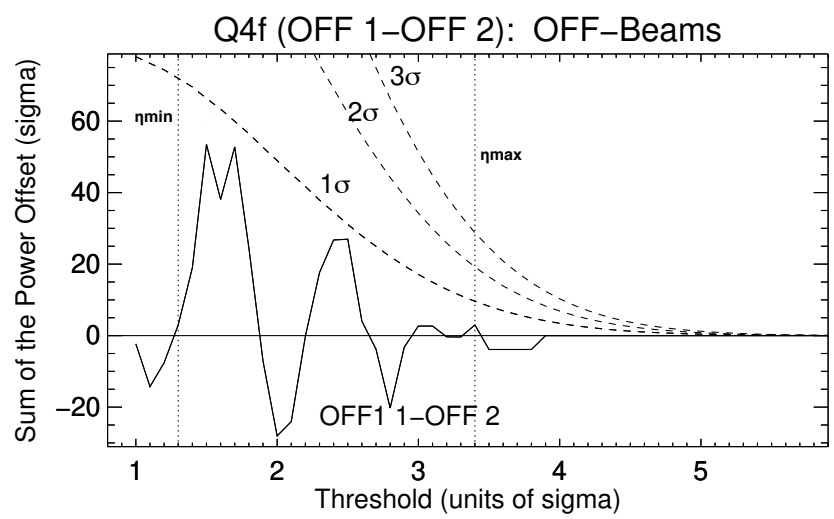

Fig. C.2. Example Q4f difference curves for Jupiter signal attenuated by a factor $\alpha=10^{-3}$. Panel $a$ : ON vs. OFF beam. Panel $b$ : OFF1 vs. OFF2 beam. The dashed lines are the $1,2,3 \sigma$ reference curves derived from computing Q4f on 10000 pairs of Gaussian noise distributions.

\section{C.4. Numerical implementation}

Based on these results, we can define a set of parameters for an automatic search algorithm. The parameter for the automated search for detections in Q1a and Q1b was $N 2>80 \%$, indicating that the ON-beam should contain more slowly variable emission than the OFF beam for most of the observation. For Q4f, N6 appears to be the most sensitive criterion for bursty signals since its ratio between the ON-OFF and OFF1-OFF2 curves is the highest. Also, N6 probes the continuous nature of the Q4 difference curve, which is essential for a detection. Therefore, we required for automatic burst detection for $\mathrm{Q} 4 \mathrm{f}$ that $N 6(\mathrm{ON}-\mathrm{OFF})$ $>1$ and $N 6(\mathrm{ON}-\mathrm{OFF})>N 6(\mathrm{OFF} 1-\mathrm{OFF} 2)$. Finally, the criterion $N 12$ is also very useful because it allows us to check the false positive rate of any tentative detection.

\section{C.5. Comparison of detection criteria for different post-processing runs}

We can plot all post-processing runs (each run is referred to as a run number) together to look for outliers and locate observations or processing runs that require a more detailed inspection. A run number is defined by a specific target (e.g., $\tau$ Boo), date and observation (e.g., L569131), frequency range (e.g., 15-38 MHz),
Table C.2. Detection criteria for Q4f from Fig. C.2.

\begin{tabular}{cccc}
\hline \hline$\#$ & ON- OFF & OFF 1 - OFF 2 & |Ratio \\
\hline N5 & 657 & 14 & 47 \\
N6 & 6.1 & 0.1 & 61 \\
N7 & 88 & 26 & 3 \\
N8 & 82 & 0 & - \\
N9a & 1.3 & 1.3 & 1.2 \\
N9b & 5.9 & 3.2 & 1.7 \\
N10 & 84 & 22 & 3.8 \\
N11 & 0 & 8 & - \\
N12 & $10^{-7}, 5 \sigma$ & $0.49,1 \sigma$ & $5 \sigma$ \\
\hline
\end{tabular}

Notes. Column 1: detection criterion. Column 2: detection criterion applied to the difference between $\mathrm{ON}$ and OFF beam. Column 3: detection criterion applied to the difference between OFF1 and OFF2 beam. Column 4: absolute value of the ratio of ON-OFF (Col. 2) by OFF1-OFF2 (Col. 3).

(a)

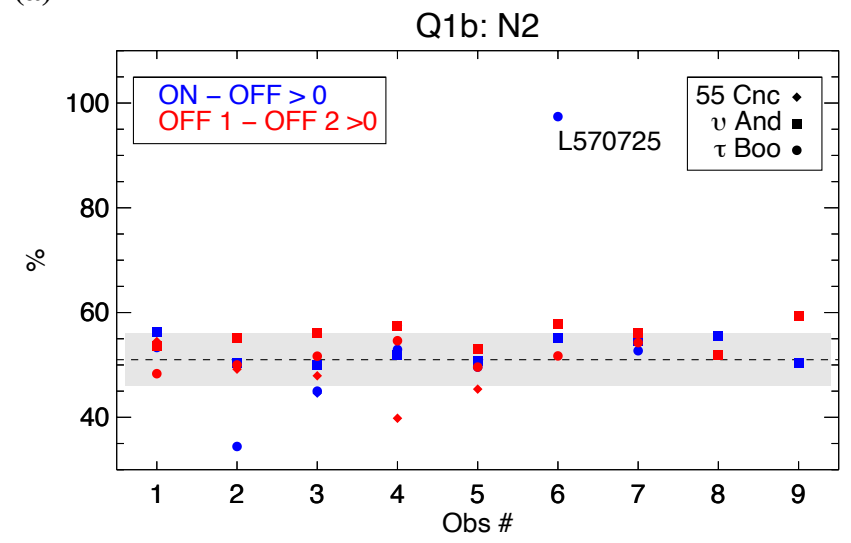

(b)

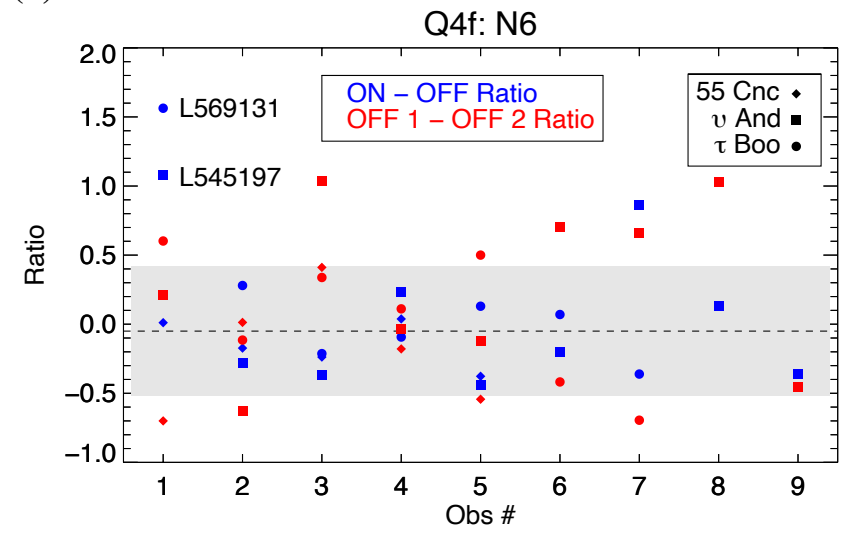

Fig. C.3. Detection criteria for all observations. Panel a: detection criterion $\mathrm{N} 2$ for the observable quantity Q1b. Panel $b$ : detection criterion N6 for the observable quantity Q4f. Only the lowest frequency band (15-38 MHz for $\tau$ Boo and $v$ And and $26-50 \mathrm{MHz}$ for $55 \mathrm{Cnc}$ ) and IVI is shown. The median value for all post-processing runs is shown as a dashed line. The $1 \sigma$ standard deviation is shown as the gray area.

polarization (e.g., $|V|)$, and rebin time ( $\delta \tau, 1 \mathrm{~s}$ or $10 \mathrm{~s})$. An example of this can be found in Fig. C.3, which shows, as a function of observation number, (a) the detection criteria N2 calculated for the observable quantity Q1b, (b) the detection criterion and N6 for the observable quantity Q4f. 
For the slowly varying emission, Fig. C.3a shows that observation L570725 of $\tau$ Boötis stands out from the other observations and requires a detailed analysis. This detailed analysis is presented in Sect. 5.1.2.

For the burst emission, Fig. C.3b shows the detection criterion N6 for the observable quantity Q4f. As for panel a, this is used to identify observations which require a detailed analysis. Figure C.3b shows two observations which stand out from the overall distribution, namely L569131 and L545197. L569131 corresponds to the $\tau$ Boo observation in which we tentatively identify burst emission (Sect. 5.1.1); L545197 is the observation of $v$ And in which we see a marginal detection (Sect. 5.2).

\section{Appendix D: Integrated spectrum (Q1b) plots for all exoplanet observations}

Low-level systematic features in the integrated spectrum $(\mathrm{Q} 1 \mathrm{~b})$ are seen in all beams for all the $55 \mathrm{Cnc}, v$ And, $\tau$ Boo observations (Fig. D.1). In the $\tau$ Boo (panel e) and $v$ And (panel c) observations we see a ripple pattern that is likely due to an instrumental effect. Since all the observations are processed identically with the BOREALIS pipeline it is unlikely that the data processing created the ripples since they are not the same in the two different data sets and do not do appear in the 55 Cnc observations.

For each individual observation, the features in the OFF1 and OFF2-beam are equivalent within the error bars as can be demonstrated by subtracting the two beams (bottom panels in the left column of Fig. D.1). We also find for each observation that the $\mathrm{ON}$ and OFF-beams are equivalent within the error bars (right column of Fig. D.1) with the exception of observation L570725 (Fig. D.1f). Therefore, any variations in the observing conditions (ionospheric and instrumental) are similar between all beams. We do observe large differences between the OFF beams of different dates showing that the systematics change between observations. However, these systematic features do not change over time in one observation, indicating that they may be caused by either an instrumental effect, by a source that is in the sidelobes, or a combination of both effects. For all observations, the lowest intensity values found are similar ( $\sim 0.003$ of the SEFD or $5.1 \mathrm{Jy}$ ) suggesting low-level instrumental noise is always present. The features on top of that are likely caused by a combination of sources in the side lobes and non-uniform instrumental effects.

Specifically, for every $\tau$ Boo observation except L570725 there is a bump in the OFF signal at $20-30 \mathrm{MHz}$. We find that ON-beam feature in observation L570725 has an amplitude comparable to the features seen in both OFF beams in all other $\tau$ Boo observations (Figs. D.1e-f). Only for observation L570725 are the 2 OFF beams lower than every other OFF beam (Fig. D.1e). However, the structure in the dynamic spectrum of the observation L570725 is not the same as in the dates with the large-scale systematics (see Appendix I). 
(a)

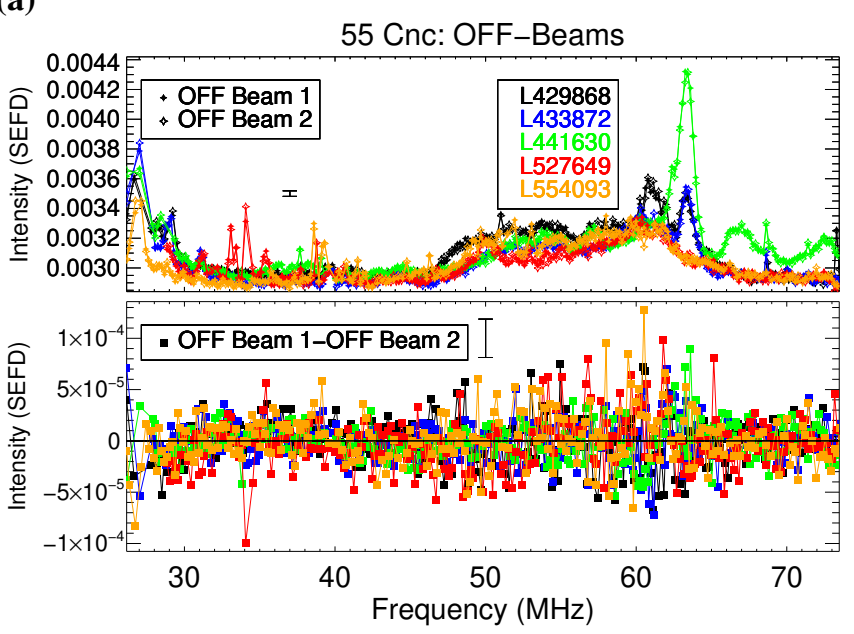

(c)

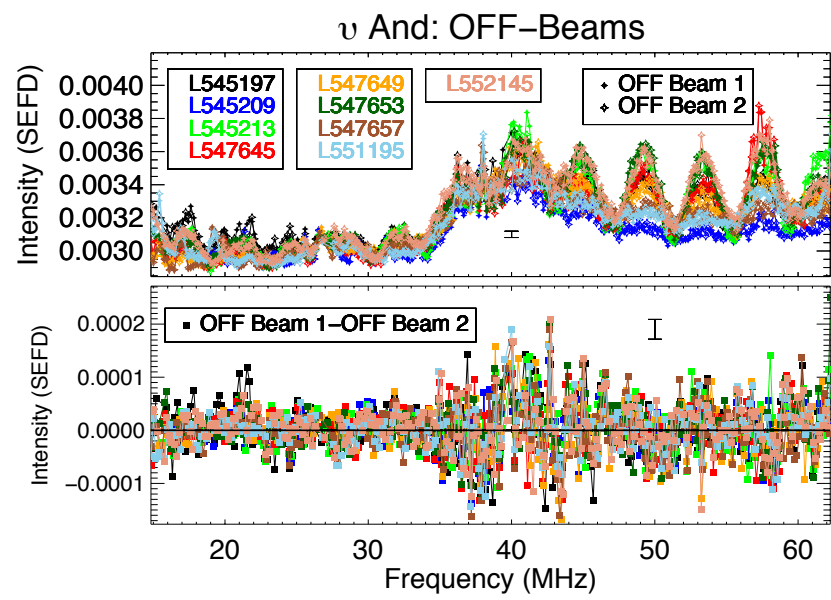

(e)

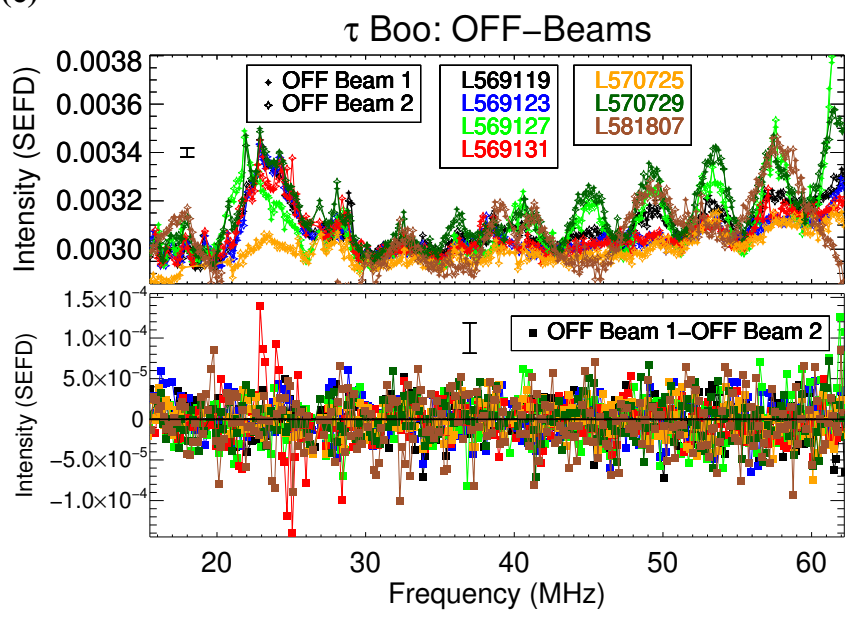

(b)

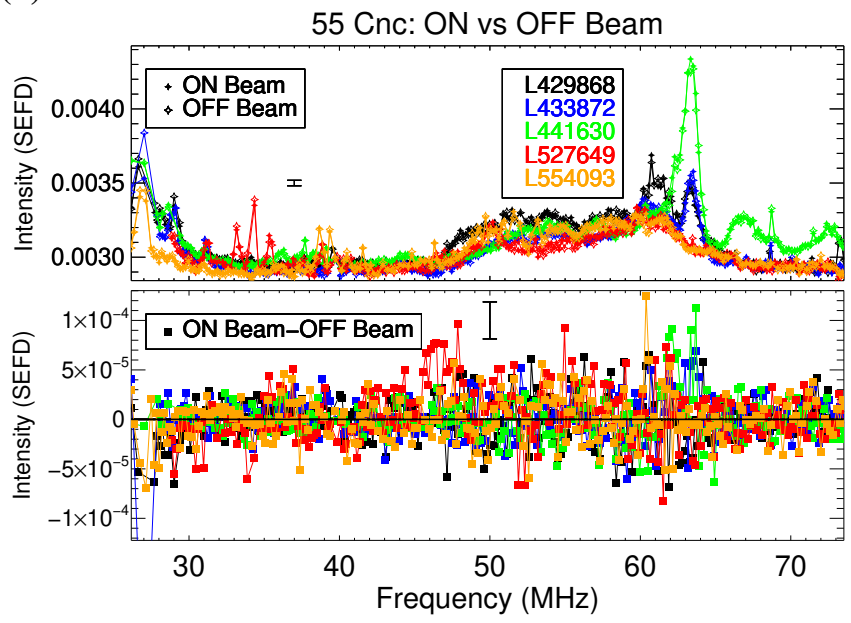

(d)

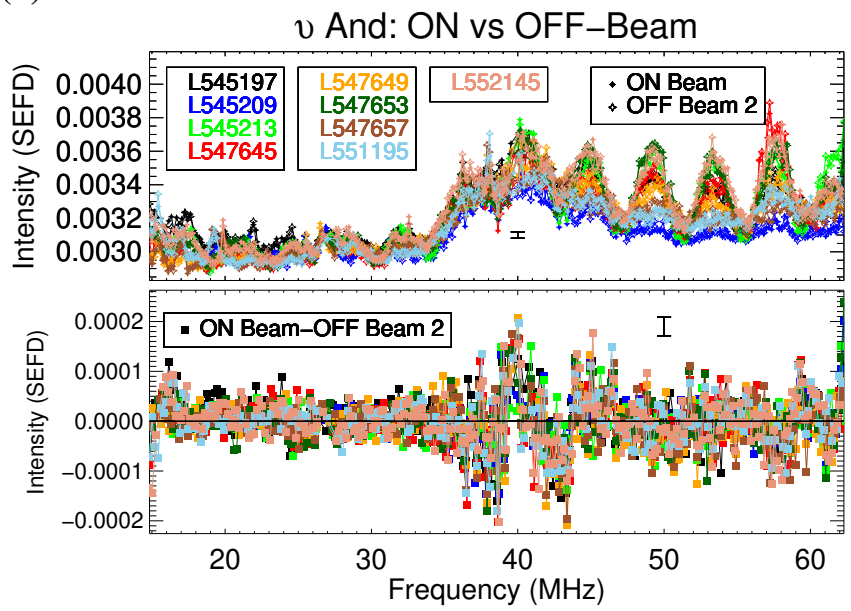

(f)

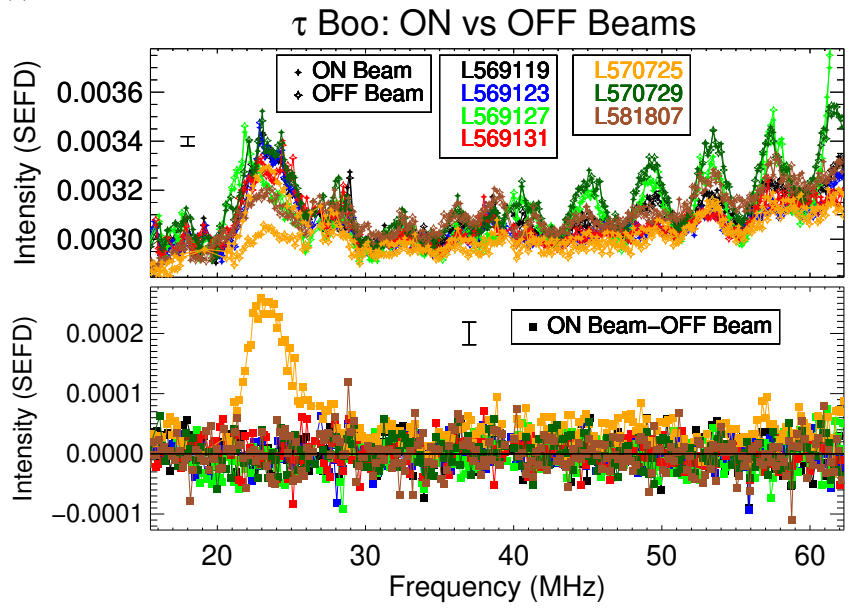

Fig. D.1. Integrated spectrum (Q1b) for all beams in all observations for $55 \mathrm{Cnc}$ (panels a and $b$ ), $v$ And (panels $c$ and $d$ ), and $\tau$ Boo (panels $e$ and $f$ ). Large scale features are seen for all dates, however, they change between observations. Panels $c-f$ : ripple pattern that is likely due to an instrumental effect (e.g., imperfect phasing). There are no large-scale differences seen between the two OFF beams (bottom plots in panels $a$, $c$, and $e$ ) for any date. In every individual observation, the two OFF-beams and ON-beam (expect L570725) are equivalent with each other within the pure Gaussian noise error bars $(\sigma=1 / \sqrt{b \tau})$. Dynamic spectrum differences of all OFF beams in every $\tau$ Boo observation can be found in Fig. I.1. The $\tau$ Boo ON-beam signal in observation L570725 (orange curve in panel $f$ ) is the only large-scale difference between the ON and OFF beams that is seen (bottom plot in all panels). The $20-30 \mathrm{MHz}$ features in many of the $\tau$ Boo b dates are the same order of magnitude as the ON-beam signal in observation L570725 (panel e). The dynamic spectrum of the ON-beam in observation L570725 subtracted by the OFF beams in the other $\tau$ Boo observations can be found in Fig. I.1. 
Appendix E: Non-detection of burst emission in observation L570725

(a)

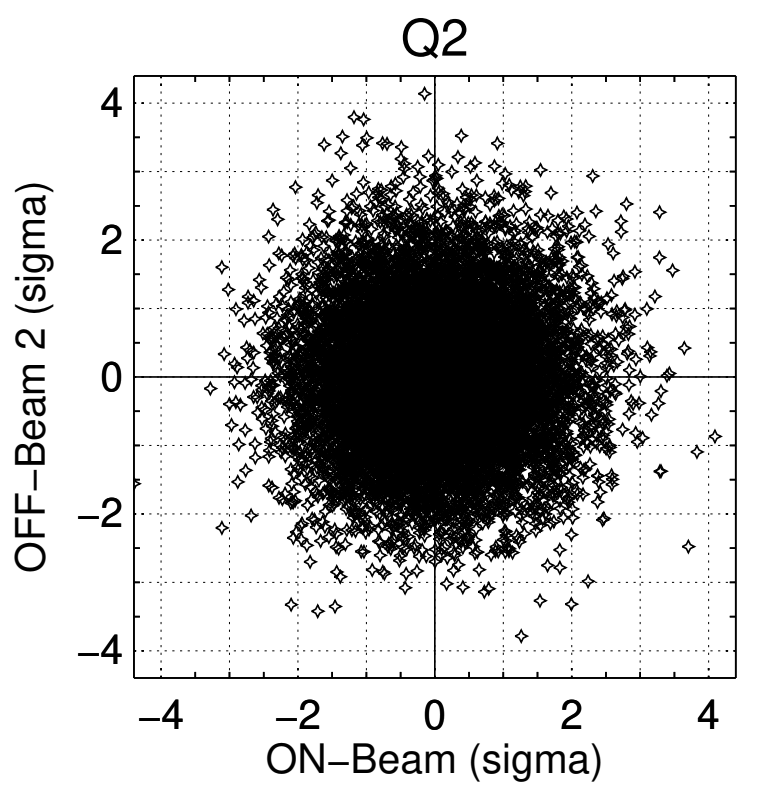

(c)

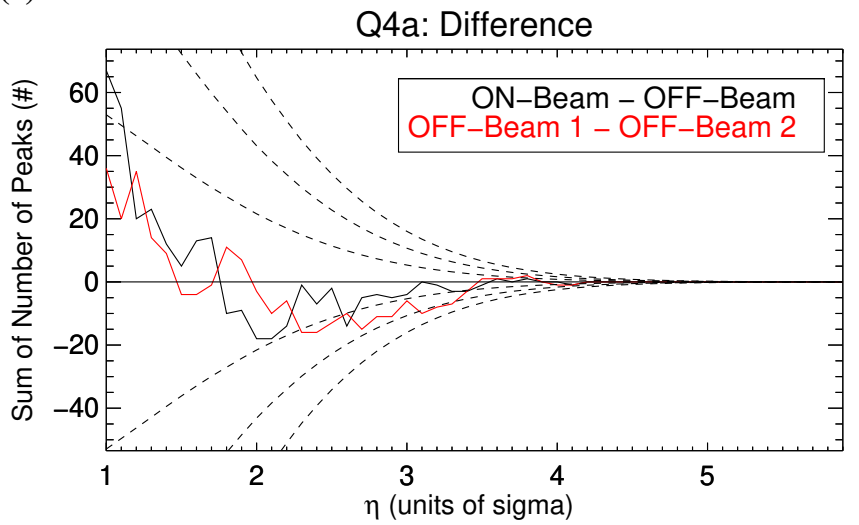

(e)

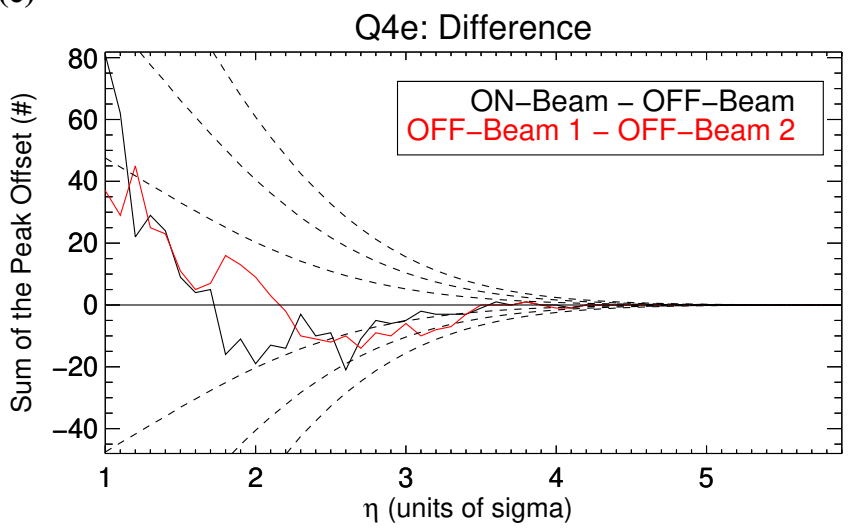

(b)

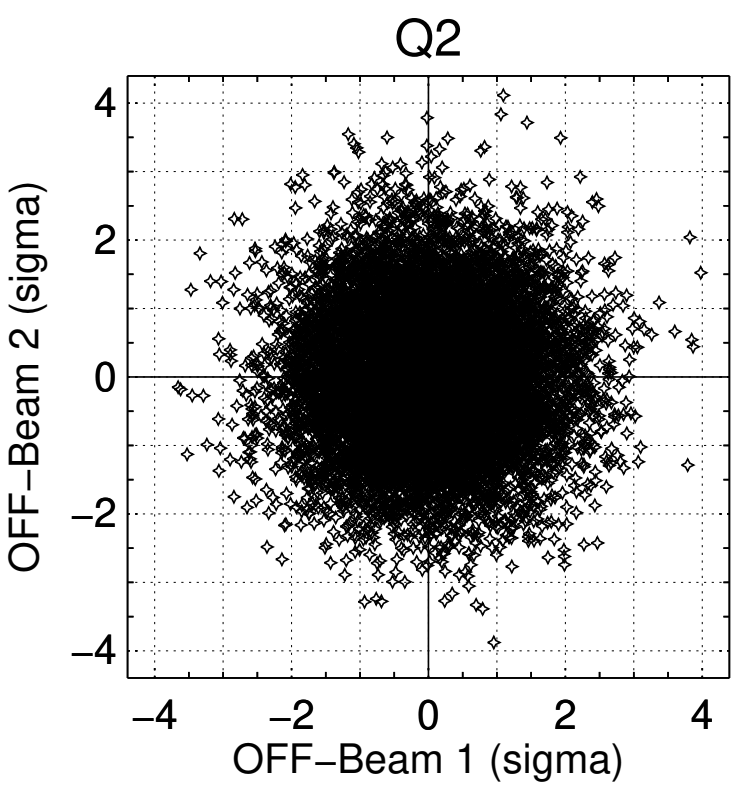

(d)

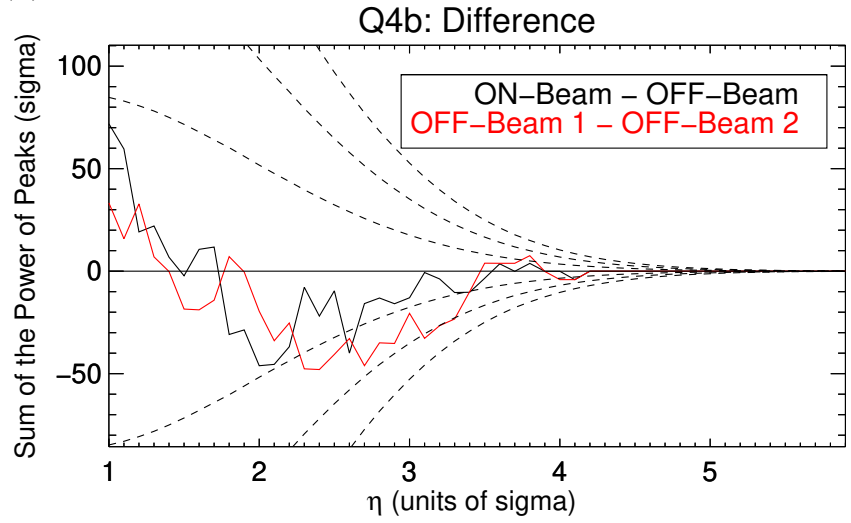

(f)

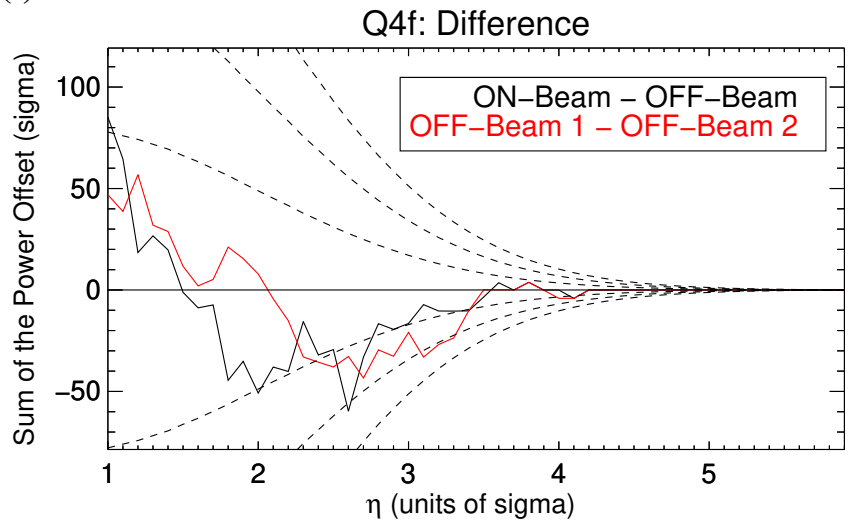

Fig. E.1. Q2 (panels $a$ and $b$ ) and beam differences for Q4a (panel c), Q4b (panel d), Q4e (panel e), and Q4f (panel f) for $\tau$ Boo in observation L570725 from the range 21-30 MHz in Stokes- $V\left(\left|V^{\prime}\right|\right)$. Panel $a$ : Q2 for the ON-beam vs the OFF-beam 2. Panel $b$ : Q2 for the OFF-beam 1 vs. the OFF-beam 2. Panel c: Q4a (number of peaks). Panel d: Q4b (power of peaks). Panel e: Q4e (peak offset). Panel $f$ : Q4f (peak offset). For panels $c-f$ the black lines are the ON-beam difference with the OFF beam 2 and the red lines are the OFF beam difference. The dashed lines are statistical limits $(1,2,3 \sigma)$ of the difference between all the $\mathrm{Q} 4$ values derived using two different Gaussian distributions (each performed 10000 times). We do not see any excess signal in the ON-beam compared to the OFF-beams. Therefore, this observation is a non-detection for burst emission. We find by performing Gaussian simulations that the probability to obtain the OFF beam curve in Q4f (panelf) is $\sim 73 \%$, whereas the probability to randomly reproduce the ON-beam curve is $\sim 82 \%$. 


\section{Appendix F: Statistical significance of slow emission detection in L570725}

To quantify the statistical significance of the detection of a slowly varying signal in observation L570725, we use a method similar to the technique outlined in Turner et al. (2019) for the significance of the Q4f detections (see also Sect. 5.1.1).

We first calculate the observable quantity Q1a for the $\mathrm{ON}$ and OFF beam. From this, we obtain the difference between both, $\mathrm{Q} 1 \mathrm{a}(\mathrm{ON})-\mathrm{Q} 1 \mathrm{a}(\mathrm{OFF})=\mathrm{Q} 1 \mathrm{a}_{\text {Diff }}$. We then normalize $\mathrm{Q} 1 \mathrm{a}_{\text {Diff }}$ by its standard deviation and calculate the average value of the normalized curves, which we denote as $\left\langle\mathrm{Q} 1 \mathrm{a}_{\text {Diff }}\right\rangle$. For observation L570725, we obtain $\left\langle\mathrm{Q} 1 \mathrm{a}_{\text {Diff }}\right\rangle=2.7$. In the same way, we define $\left\langle\mathrm{Q} 1 \mathrm{~b}_{\text {Diff }}\right\rangle$ and find $\left\langle\mathrm{Q} 1 \mathrm{~b}_{\text {Diff }}\right\rangle=8.2$.

These values are compared to those obtained in the case when both beams only contain random Gaussian noise. We generated a random distribution of points for the $\mathrm{ON}$ and OFF beams (generating an artificial dynamic spectrum with the same number of points) and calculated $\left\langle\mathrm{Q} 1 \mathrm{a}_{\text {Diff }}\right\rangle$ and $\left\langle\mathrm{Q} 1 \mathrm{~b}_{\text {Diff }}\right\rangle$. We generated over $10^{6}$ instances of this artificial data set. In none of these simulated cases, the value of $\left\langle\mathrm{Q} 1 \mathrm{a}_{\text {Diff }}\right\rangle$ and $\left\langle\mathrm{Q} 1 \mathrm{~b}_{\text {Diff }}\right\rangle$ reached the values obtained in observation L570725. By interpolating the peak values obtained for $\left\langle\mathrm{Q} 1 \mathrm{a}_{\text {Diff }}\right\rangle$ and $\left\langle\mathrm{Q} 1 \mathrm{~b}_{\text {Diff }}\right\rangle$ over a set of $N$ simulations (with $N$ between $10^{5}$ and $10^{6}$ ), we find that the probability to randomly obtain a signal as strong as the observed one is $2.1 \times 10^{-12}$ for $\mathrm{Q} 1 \mathrm{a}_{\text {Diff }}$ and $1 \times 10^{-18}$ for $\mathrm{Q} 1 \mathrm{~b}_{\text {Diff. This false }}$ positive rate corresponds to a statistical significance of $6.9 \sigma$ and $8.6 \sigma$, respectively.

As a final step, we compare this value to those obtained for the OFF beams. In that case, we find that the false positive rate is $\sim 90 \%$ for $\mathrm{Q} 1 \mathrm{a}(\mathrm{OFF} 1)-\mathrm{Q} 1 \mathrm{a}(\mathrm{OFF} 2)$ and $\sim 100 \%$ for $\mathrm{Q} 1 \mathrm{~b}(\mathrm{OFF} 1)-\mathrm{Q} 1 \mathrm{~b}(\mathrm{OFF} 2)$. Therefore, the OFF beams difference clearly corresponds to a non-detection.

We note that this procedure assumes all points in the Q1a and Q1b curves are uncorrelated. In the case of instrumental effects, sources in the side lobe or other systematic errors this assumption does not hold.

\section{Appendix G: Pulsar B0809+74 observation L570723}

\section{G.1. Replica signal in OFF-beam}

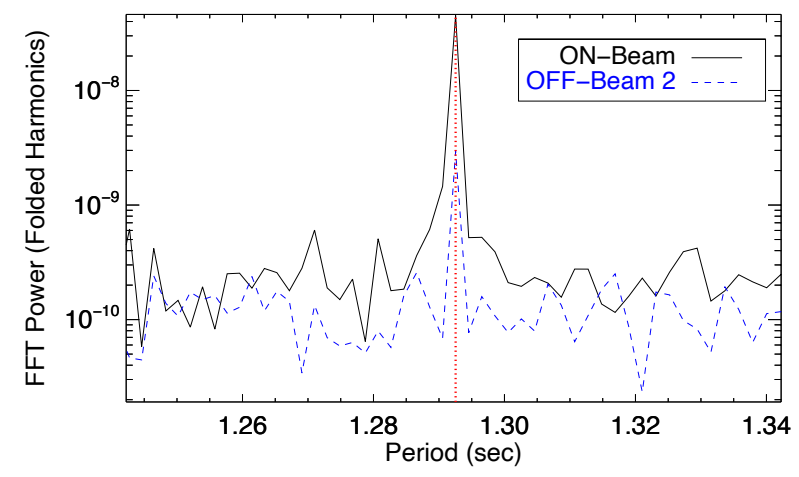

Fig. G.1. FFT of the pulsar B0809+74 (observation L570723) for the ON-beam (black-line) and OFF-beam 2 (dashed blue line). The known period of the pulsar is marked as a dashed red line.

The observation of the pulsar B0809+74 can be used to independently study the possible systematics (e.g imperfect phasing and low-level noise) affecting the $\tau$ Boo observation L570725

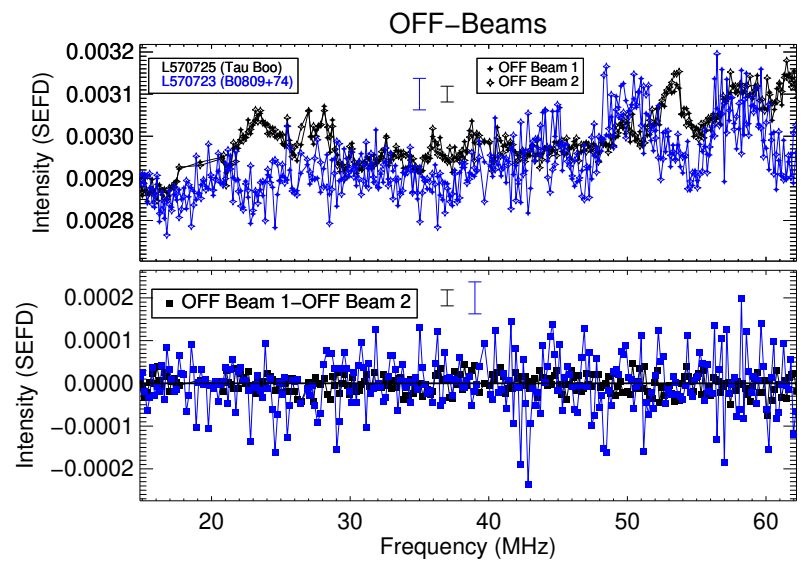

Fig. G.2. Integrated spectrum (Q1b) for the OFF beams on $\tau$ Boo (observation L570725) and the pulsar B0809+74 (observation L570723, taken 15 min before observation L570725). For observation L570725 only the first 15 min of data were used to calculate Q1b to allow for a more consistent comparison with the pular observation. The two observations have similar features in Q1b and above $30 \mathrm{MHz}$ are consistent with each other assuming Gaussian error bars. We performed a K-S test on the two curves above $30 \mathrm{MHz}$ and find that the probability that the two curves are drawn from the same parent distribution (the null hypothesis) is $97 \%$. The fact that the two beams are pointed at completely different parts of the sky and still have similar overall flux levels suggests that the majority of the signal in the beams is instrumental. The $20-30 \mathrm{MHz}$ feature in the OFF beam of $\tau$ Boo observation L570725 is the faint signal replicated from the ON-beam (Sect. 5.1.2; Fig. 6).

(Sect. 5.1.2). The pulsar observation L570723 was taken $15 \mathrm{~min}$ before observation L570725. In order to detect B0809+74, we use the same procedure as in Turner et al. (2017a). The FFT was performed on the data after running it through the BOREALIS pipeline and de-dispersing the observations at the known dispersion measure. To get the final power spectrum in the FFT, the 6 first harmonics were folded together. The FFT was computed from the range $30-55 \mathrm{MHz}$. The FFT of the ON-beam, OFF-beam 1, and OFF-beam 2 are shown in Fig. G.1.

We find that the pulsar is detected with a signal-to-noise ratio $\left(\mathrm{SN}_{\mathrm{FFT}}\right)$ of $\sim 628$ in the ON-beam and with $\mathrm{SN}_{\mathrm{FFT}}$ of $\sim 23$ and $\sim 57$ in the OFF beams, respectively. This result confirms that a replica signal of the ON-beam can appear in the OFF beam, as was also found in the $\tau$ Boo observation L570725 (Sect. 5.1.2; Fig. 6).

\section{G.2. Comparison of integrated spectra (Q1b)}

We also compare the integrated spectra (observable quantity Q1b) of one of the observations of the pulsar B0809+74 to those of the $\tau$ Boo observation. Figure G.2 shows Q1b for both OFF beams for the two observations (B0809+74 observation L570723 and $\tau$ Boo observation L570725). We only used the first 15 minutes of data from the $\tau$ Boo L570725 observation to derive Q1b to allow for a more consistent comparison. Above $30 \mathrm{MHz}$, the two observations are consistent with each other within error bars. Additionally, we performed a K-S statistical test on the two curves above $30 \mathrm{MHz}$ and find that the probability that the two curves are drawn from the same parent distribution (the null hypothesis) is $97 \%$. In the frequency range $20-30 \mathrm{MHz}$, the $\tau$ Boo observation shows large-scale features in both OFF beams; this feature is absent in the pulsar observation. Therefore, the likely source of the $21-30 \mathrm{MHz}$ feature in the ON-beam of the $\tau$ Boo observation L570725 (Fig. 5b) is either excess flux in the beam or an unknown time-dependent instrumental effect. 
Appendix H: Station inspection plots
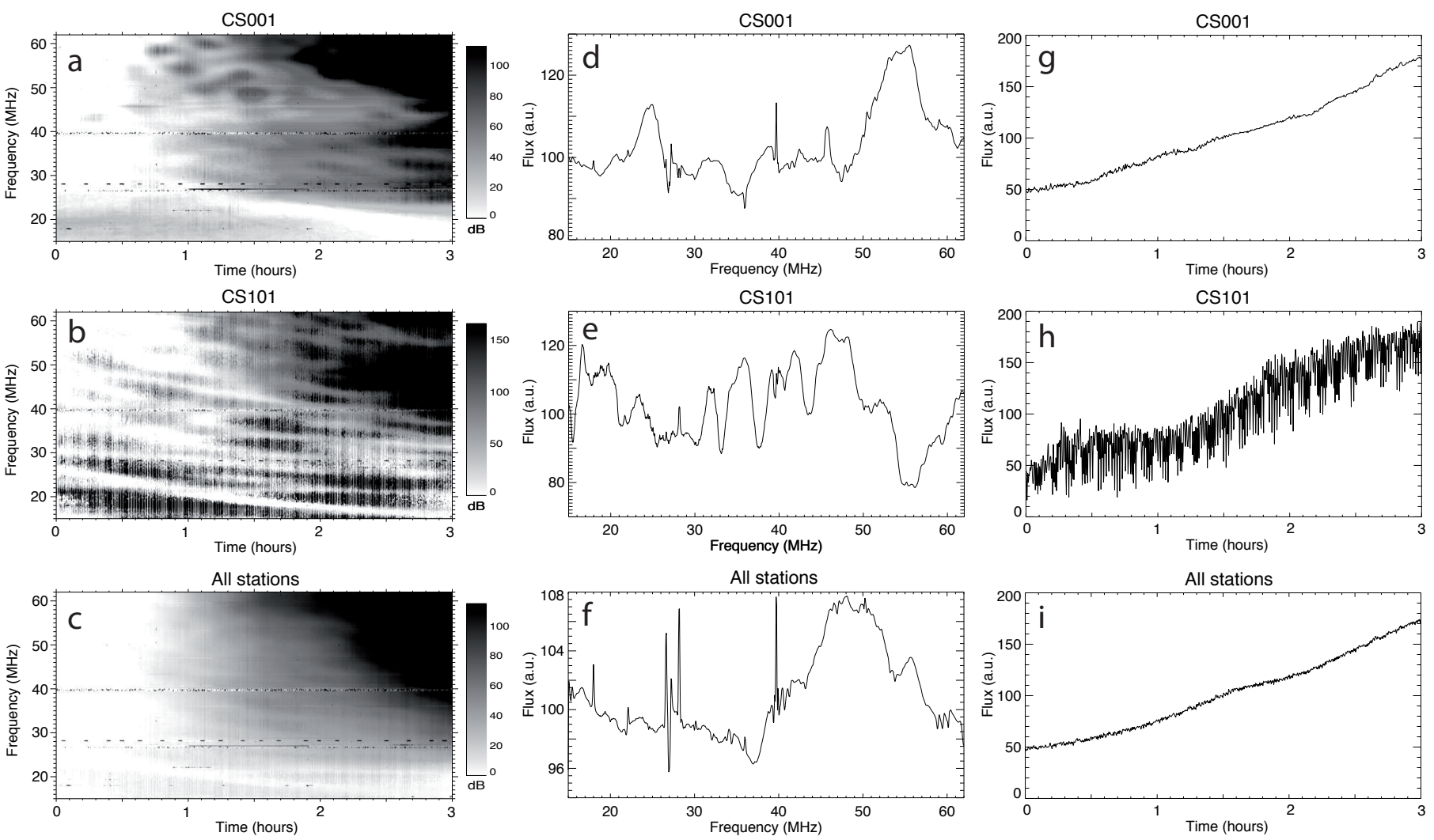

Fig. H.1. Analysis of the station inspection plots for observation L570725. Top row: good station CS001 (panels a, d, g). Middle row: bad station CS101 (panels b, e, h). Bottom row: incoherent (not phased) sum of all stations. Left column (panels a-c): station inspection plots. Each plot has been normalized by an average background. Large scale features are seen foe the bad station CS101. Middle column (panels $d-f)$ : integrated spectra (Q1b) derived from the station inspection plots. Right column (panels $g-i$ ): time-series (Q1a) derived from the station inspection plots. No large bumps are seen within 20-30 MHz for the bad station (CS101), therefore they do not seem to be cause of the L570725 ON-beam signal seen in Fig. 5.

During each observation, station summary plots are produced by ASTRON to monitor telescope stability and data quality. We examined these station inspection plots to search for a possible non-planetary cause of the signal seen in the ON-beam of observation L570725. We discovered that in each of our LOFAR observations at least one station was misbehaving (summarised in Table A.1).

In Fig. H.1, we show a few examples for station inspection plots (left-hand panels) and some derived quantities for the $\tau$ Boo observation L570725. We compare a good station (CS001), a bad station (CS101), and the incoherent sum of all stations.

To directly compare to the observed signal, we digitized the dynamic spectra and calculated the integrated spectrum (Q1b, middle panels) and time-series (Q1a, right-hand panels) for the stations mentioned above. We do not see any large-scale bumps in the range $20-30 \mathrm{MHz}$ in Q1b in any of the good or bad stations and also when we combine all 24 stations together (this is an incoherent sum since the phases were not taken into account). Our findings suggest that the spurious behavior seen in the stations plots are not likely the origin of the detected signal discussed in Sect. 5.1.2.

\section{Appendix I: Dynamic spectrum differences of the $\tau$ Boo observations}

We compare the dynamic spectrum of the ON-beam of observation L570725 to the OFF beams of all the other $\tau$ Boo observations to determine the structure of the emission. Figure I.1 shows the subtraction of the ON-beam of L570725 by the different OFF beams. For all panels in this figure, the $x$-axis is the observation UT time subtracted by the transit time of the meridian of $\tau$ Boo. Moving to this reference frame is important because it ensures that observations taken at different dates will have the same beam characteristics (elevation, main beam pattern, and side lobes) at each time step in the new frame. The only differences between beams should be ionospheric variations, differences in instrumental systematics, and any remaining low-level RFI. In most cases, a structured feature persists. Therefore, the ON-beam signal in observation L570725 is inherently different than all OFF beams for every $\tau$ Boo observation. Hence, even though the integrated spectra of the different dates may look similar (Fig. D.1), their actual emission (and thus their emission sources) is not the same. In the right column of Fig. I.1, we also show the difference of the OFF beam dynamic spectrum for each date. As with the integrated spectra (Fig. D.1), the dynamic spectrum for the two OFF beams in each individual date are very similar and only random noise remains. 
(a)

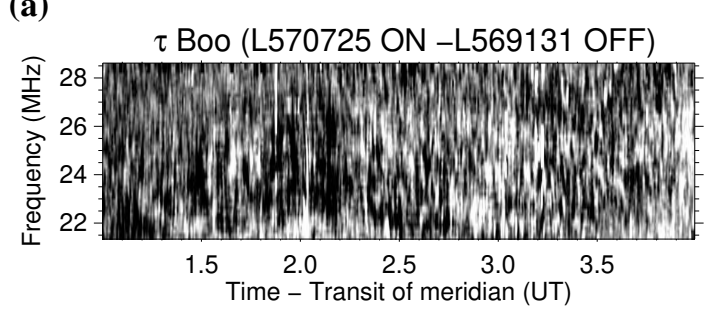

(c)

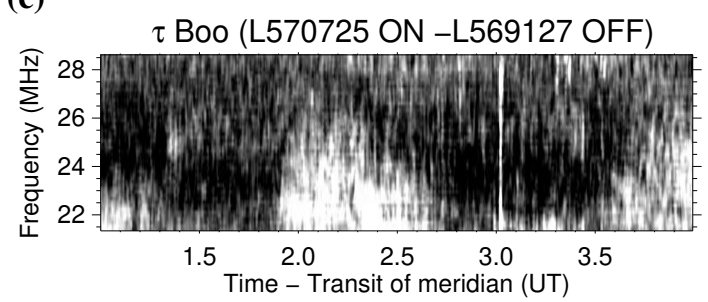

(e)

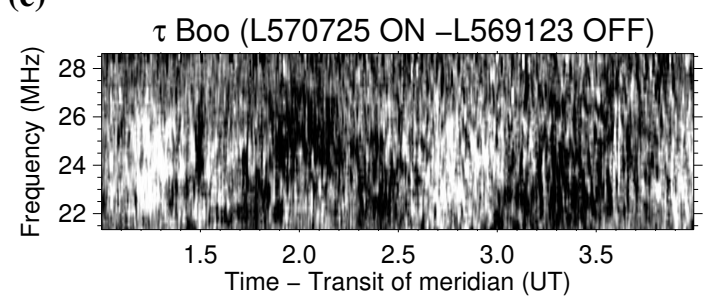

(g)

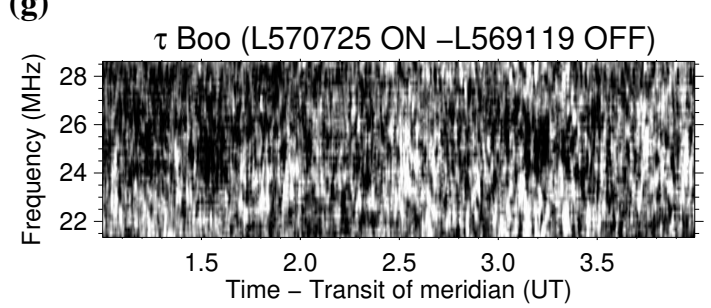

(i)

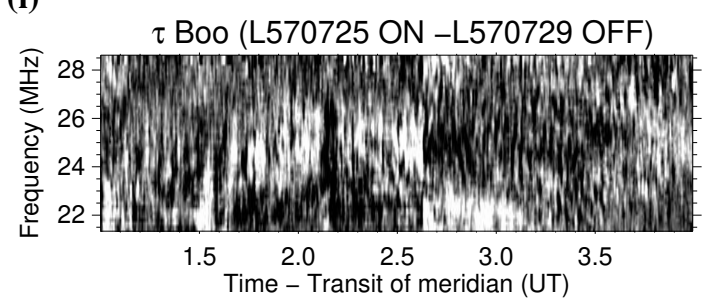

(k)

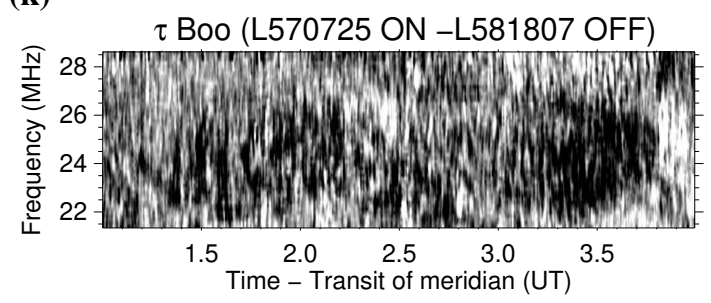

(b)
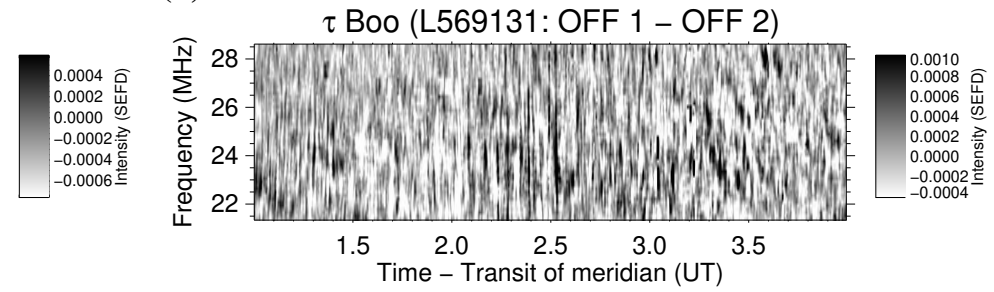

(d)
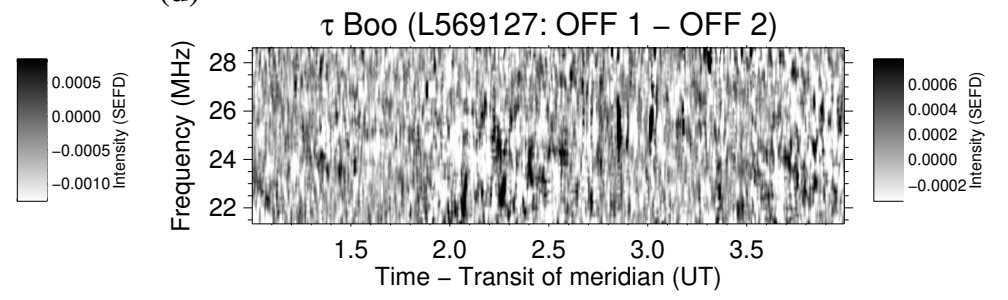

(f)
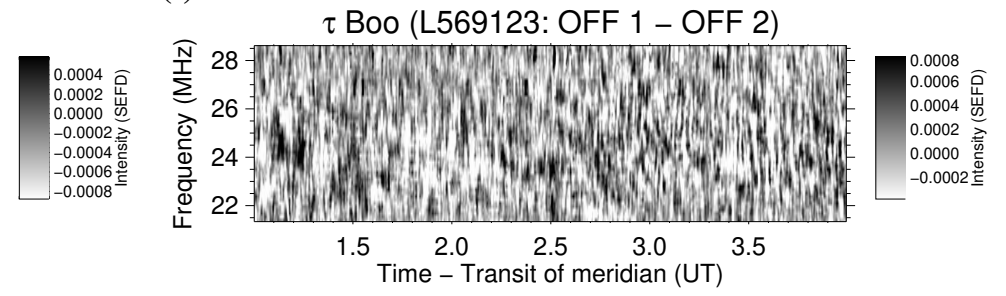

(h)
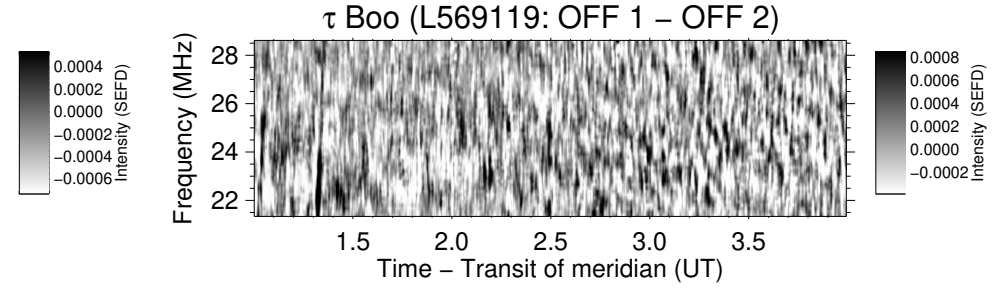

(j)
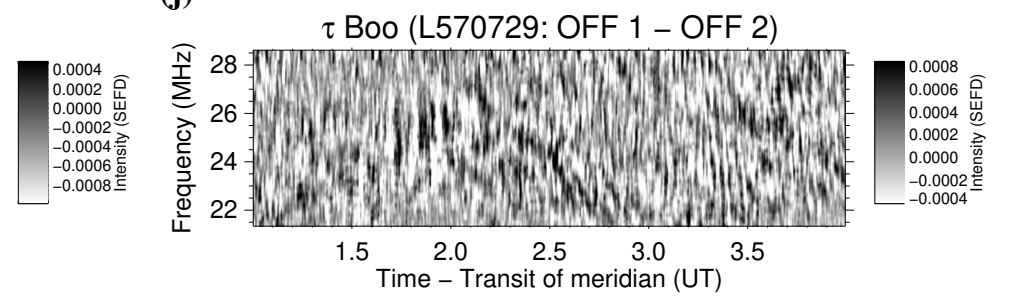

(l)
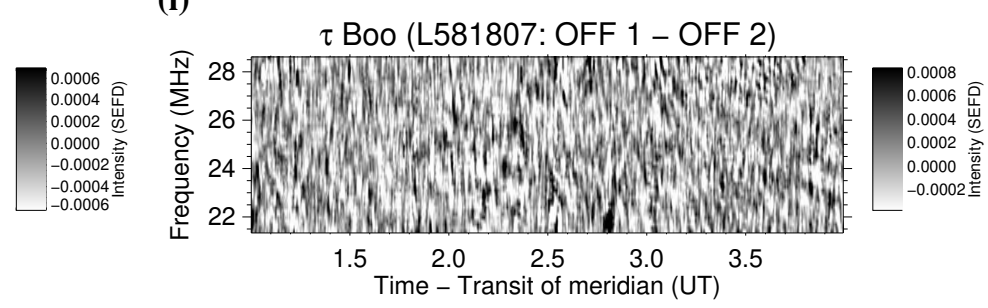

Fig. I.1. Dynamic spectrum differences of $\tau$ Boo in Stokes- $V\left(\left|V^{\prime}\right|\right)$ in the ON-beam in L570725 with the OFF beams in L569131 (panel a), L569127 (panel c), L569123 (panel e), L569119 (panel g), L570729 (panel i), and L581807 (panel $k$ ). Dynamic spectrum differences of the two OFF beams for each date can be found in the right column (panels $b, d, f, h, j$, and $l$ ). The data from the range $21-29 \mathrm{MHz}$ is shown. The $x$-axis for all plots is the UT time of the observation subtracted by the transit time of the meridian. Moving to this reference frame ensures that even though the observations were taken at different dates, the characteristics of the beams (elevation, main beam pattern, and side lobes) at each time step are exactly the same. Thus, ionospheric variations, differential instrumental effects, and any remaining low-level RFI are the only difference between beams. The structured differences between the ON and OFF beams suggest that the signal in L570725 does not have the same time-frequency characteristics as the features seen in OFF beams of all the other dates. We do not see any obvious structure in the OFF beam difference plots. This suggests that for each observation the OFF beams are similar in their time-frequency emission structures. We find a consistent conclusion with what we find when comparing Q1b (integrated spectrum) for the two OFF beams for each individual observation (Fig. D.1). 
Appendix J: $v$ Andromedae marginal signal

(a)

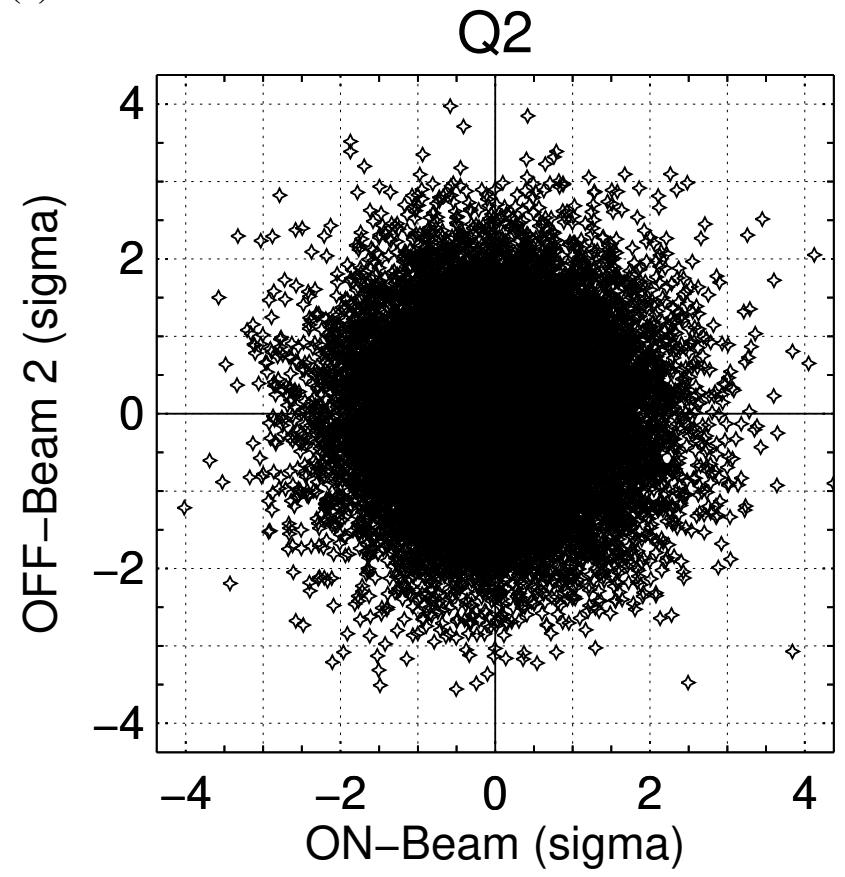

(c)

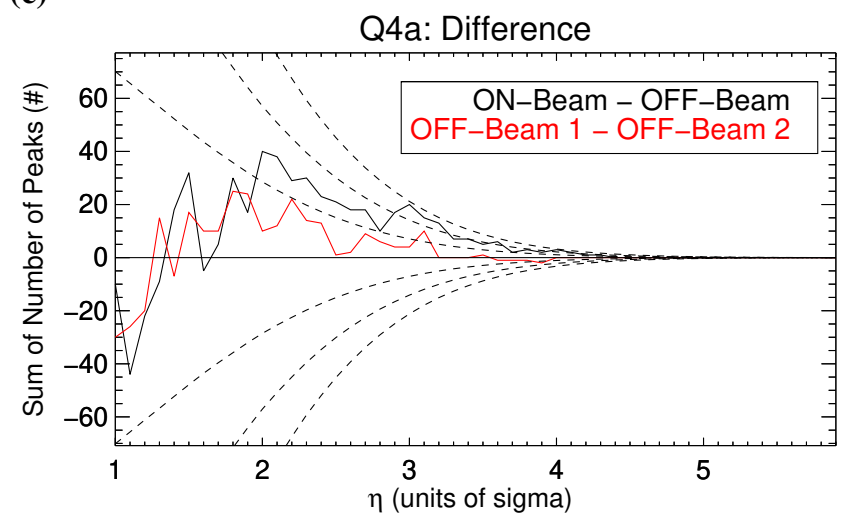

(e)

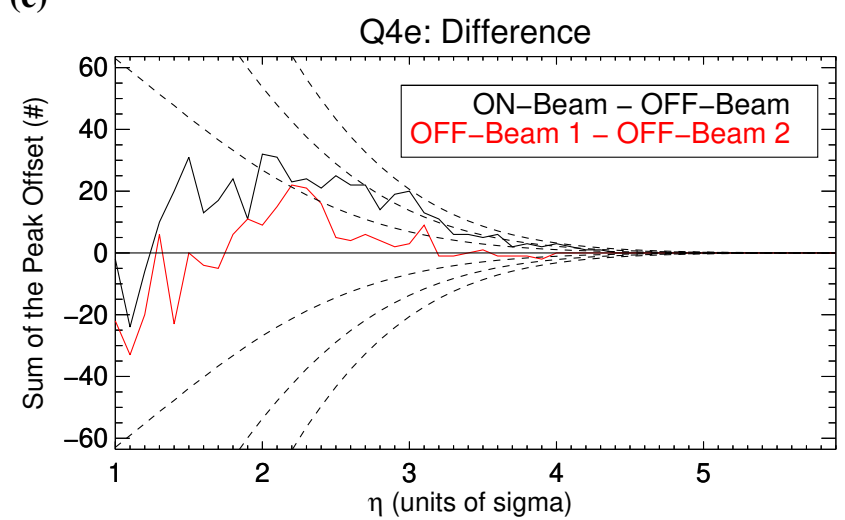

(b)

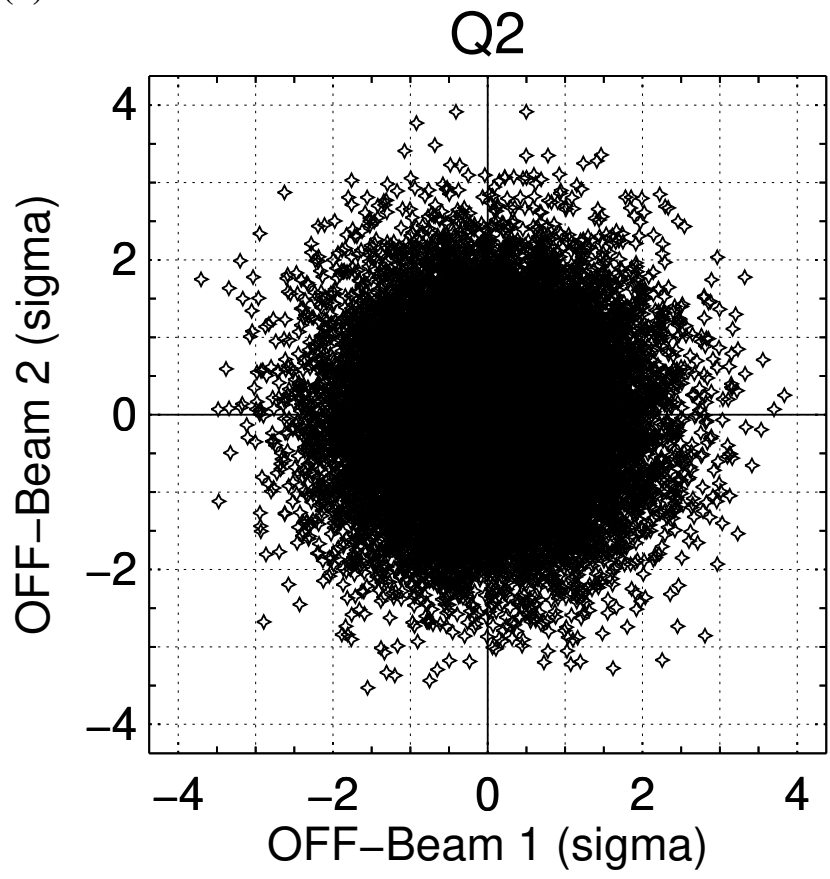

(d)

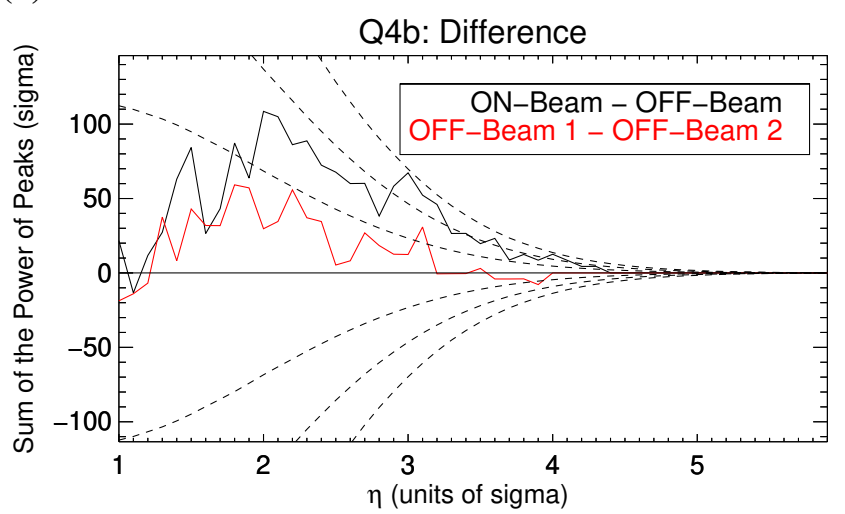

(f)

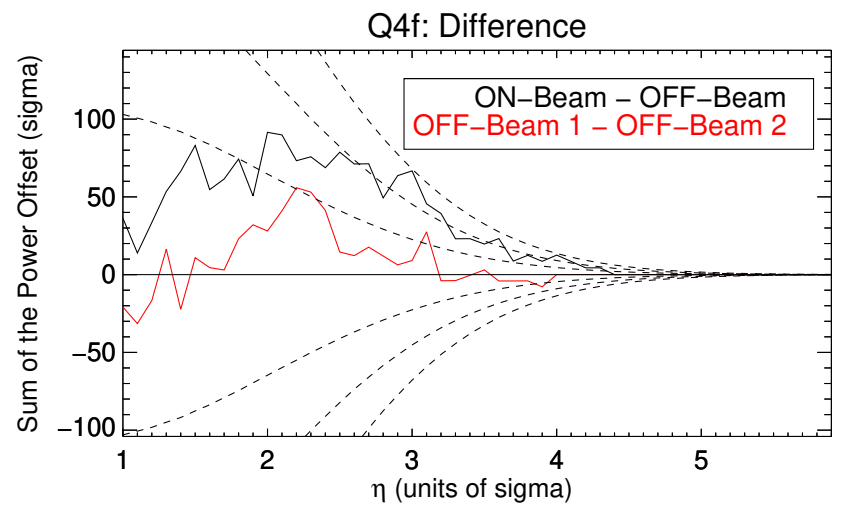

Fig. J.1. Q2 (panels $a$ and $b$ ) and beam differences for Q4a (panel c), Q4b (panel d), Q4e (panel e), and Q4f (panelf) for $v$ And in observation L545197 in the range 14-38 MHz in Stokes- $V\left(\left|V^{\prime}\right|\right)$. The tentative signal is most clearly seen in Q4f, which is distinctly different for the ON-beam (black curve) than for the OFF beams (red curve). The other comments are the same as Fig. 4. The probability to reproduce the ON-beam curve by chance is $1.3 \%$ or $2.2 \sigma$, whereas the false-positive probability for the OFF beams is $59 \%$. Additionally, we performed the Kolmogorov-Smirnov statistical test on the two curves for Q4f in panel $f$ and find that the probability to reject the null hypothesis (that the two curves are drawn from the same parent distribution) is $76 \%$. Therefore, the signal is possibly a false-positive. 